Florida International University FIU Digital Commons

$3-25-2008$

\title{
Anti-Quorum Sensing Agents from South Florida Medicinal Plants and their Attenuation of Pseudomonas Aeruginosa Pathogenicity
}

Allison L. Adonizio

Florida International University

DOI: $10.25148 /$ etd.FI08081501

Follow this and additional works at: https://digitalcommons.fiu.edu/etd

Part of the Biology Commons

\section{Recommended Citation}

Adonizio, Allison L., "Anti-Quorum Sensing Agents from South Florida Medicinal Plants and their Attenuation of Pseudomonas Aeruginosa Pathogenicity" (2008). FIU Electronic Theses and Dissertations. 13.

https://digitalcommons.fiu.edu/etd/13 


\section{FLORIDA INTERNATIONAL UNIVERSITY}

Miami, Florida

\section{ANTI-QUORUM SENSING AGENTS FROM SOUTH FLORIDA MEDICINAL PLANTS AND THEIR ATTENUATION OF PSEUDOMONAS AERUGINOSA PATHOGENICITY}

A dissertation submitted in partial fulfillment of the requirements for the degree of DOCTOR OF PHILOSOPHY in BIOLOGY by

Allison L. Adonizio 
To: Dean Kenneth Furton

College of Arts and Sciences

This dissertation, written by Allison L. Adonizio, and entitled Anti-quorum sensing agents from south Florida medicinal plants and their attenuation of Pseudomonas aeruginosa pathogenicity, having been approved in respect to style and intellectual content, is referred to you for judgment.

We have read this dissertation and recommend that it be approved.

John Makemson

Kelsey Downum

Bradley C. Bennett

Eric Crumpler

Kalai Mathee, Major Professor

Date of Defense: March 25, 2008

The dissertation of Allison L. Adonizio is approved.

Dean Kenneth Furton

College of Arts and Sciences

Dean George Walker

University Graduate School

Florida International University, 2008 


\section{DEDICATION}

I dedicate this thesis to my father, Robert J. Adonizio. A teacher by profession, he was fundamental in my interest in science. Without his guidance, support, and most of all love, the completion of this work would not have been possible. 


\section{ACKNOWLEDGMENTS}

With the utmost gratitude, I would like to acknowledge all the kind souls essential to the completion of this dissertation. First and foremost I would like to thank my major professor Dr. Kalai Mathee, and my committee members: Bradley Bennett, Kelsey Downum, John Makemson, and Eric Crumpler for their teaching, guidance, and constructive criticism throughout the years. I am grateful for my fantastic colleagues in the labs of both Dr. Mathee and Dr. Frederick Ausubel (Harvard/MGH) especially Lisa Schneper and Natalie Maricic who have helped me immensely with the endless formalities that go along with a dissertation. Additionally, I would like to recognize Hanna Milewikz, Tomo Kawate, Monica Pupo, and Jessica Dawiaty at Harvard/MGH for holding my hand through the elucidation and characterization of active compounds, and to Steven Casper for answering my crazed 3AM phone calls about separation technique. I appreciate the financial support of the FIU MBRS-RISE program (R25 GM61347), the National Institute of Health (NIH), National Center for Complimentary and Alternative Medicine (1-T32-AT01060-01), and the Cystic Fibrosis Foundation (ADONIZ06H0/07HO/08HO). I am grateful to my friends, family, and loved ones who put up with me through long hours, limited budget, and less than pleasant responses to any questions regarding completion of this work. Finally, I would like to thank Sebastian (Felis catus), and the late Niles Atreyu, for the difficult task of holding down any and all papers on my floor...the letter $\pi$, and the number 22 . 


\title{
ABSTRACT OF THE DISSERTATION \\ ANTI-QUORUM SENSING AGENTS FROM SOUTH FLORIDA MEDICINAL \\ PLANTS AND THEIR ATTENUATION OF PSEUDOMONAS AERUGINOSA \\ PATHOGENICITY \\ by
}

\author{
Allison L. Adonizio \\ Florida International University, 2008 \\ Miami, Florida \\ Professor Kalai Mathee, Major Professor
}

With the difficulty in treating recalcitrant infections and the growing resistance to antibiotics, new therapeutic modalities are becoming increasingly necessary. The interruption of bacterial quorum sensing (QS), or cell-cell communication is known to attenuate virulence, while limiting selective pressure toward resistance. This study initiates an ethnobotanically-directed search for QS inhibiting agents in south Florida medicinal plants. Fifty plants were screened for anti-QS activity using two biomonitor strains, Chromobacterium violaceum and Agrobacterium tumefaciens. Of these plants, six showed QS inhibition: Conocarpus erectus L. (Combretaceae), Chamaecyce hypericifolia (L.) Millsp. (Euphorbiaceae), Callistemon viminalis (Sol.ex Gaertn.) G. Don (Myrtaceae), Bucida burceras L. (Combretaceae), Tetrazygia bicolor (Mill.) Cogn. (Melastomataceae), and Quercus virginiana Mill. (Fagaceae). These plants were further examined for their effects on the QS system and virulence of Pseudomonas aeruginosa, an intractable opportunistic pathogen responsible for morbidity and mortality in the immunocompromised patient. C. erectus, B. buceras, and C. viminalis were found to 
significantly inhibit multiple virulence factors and biofilm formation in this organism. Each plant presented a distinct profile of effect on QS genes and signaling molecules, suggesting varying modes of action. Virulence attenuation was observed with marginal reduction of bacterial growth, suggesting quorum quenching mechanisms unrelated to static or cidal effects. Extracts of these plants were also investigated for their effects on P. aeruginosa killing of the nematode Caenorhabditis elegans. Results were evaluated in both toxin-based and infection-based assays with P. aeruginosa strains PA01 and PA14. Overall nematode mortality was reduced $50-90 \%$. There was no indication of host toxicity, suggesting the potential for further development as anti-infectives. Using lowpressure chromatography and HPLC, two stereoisomeric ellagitannins, vescalagin and castalagin were isolated from an aqueous extract of $C$. erectus. Structures were confirmed via mass spectrometry and NMR spectroscopy. Both ellagitannins were shown to decrease signal production, QS gene expression, and virulence factor production in $P$. aeruginosa. This study introduces a potentially new therapeutic direction for the treatment of bacterial infections. In addition, this is the first report of vescalagin and castalagin being isolated from C. erectus, and the first report of ellagitannin activity on the QS system. 


\section{TABLE OF CONTENTS}

Chapter 1: Introduction....................................................

$1.1 \quad$ Preface........................................................... 2

1.2 Ethnobotany and pharmacognosy: Looking to the past for solutions of the future..................................................... 2

1.2.1 Advantages of a directed search.......................... 3

1.2.2 Linking traditional and modern medicine.................... 5

1.2.3 The Ecology and ethnobotany of southern Florida............... 6

$1.3 \quad$ Botanicals as anti-bacterial therapies............................... 9

1.3.1 Tea tree oil................................................ 9

1.3.2 Oregano oil................................................ 10

1.3.3 Myrrh.............................................. 12

1.3.4 Untapped potential for antimicrobial activity ................ 13

1.4 Quorum sensing: a novel target................................... 14

1.4.1 Anti-quorum sensing................................... 17

1.4.1.1 Mechanisms of QS inhibition.................... 19

$1.5 \quad$ Model Organism: Pseudomonas aeruginosa ............................ 21

1.5.1 Virulence factors and toxins................................ 22

1.5.2 Pseudomonas aeruginosa disease association................... 23

1.5.3 Pseudomonas aeruginosa and cystic fibrosis.................. 26

1.5.4 Current treatment protocols................................ 27

1.5.5 Antibiotic resistance in Pseudomonas aeruginosa............... 29

1.5.5.1 Biofilm formation................................ 31

1.5.5.2 Restricted surface accessibility.................. 32

1.5.5.3 Exclusion via efflux pumps..................... 33

1.5.5.4 Enzymatic inactivation of antibiotics.............. 34

1.5.5.5 Alteration of target proteins...................... 37

1.5.6 Pseudomonas aeruginosa QS specifics....................... 37

1.6 Infection Model: Caenorhabditis elegans.............................. 41

$1.7 \quad$ Main hypotheses and aims of this project............................ 43

Chapter 2: Anti-quorum sensing activity of medicinal plants in southern Florida. 44

$2.1 \quad$ Abstract.......................................................... 45

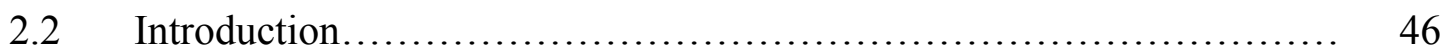

2.3 Materials and methods........................................... 49

2.3.1 Collection of plant material and extract preparation............. 49

2.3.2 Biomonitor organisms........................................ 55

2.3.3 Bioassays............................................... 55

2.3.4 Disk diffusion method......................................... 56 
$2.4 \quad$ Results....................................................... 56

2.4.1 Preliminary screen to determine effect of surface microbes and processing............................................... 56

2.4.2 Anti-quorum sensing activities in southern Florida plants using C. violaceum assays...................................... 58

2.4.3 Anti-quorum sensing activities in southern Florida plants using an $A$. tumefaciens assay.................................. 58

2.4.4 Antibacterial vs. anti-quorum sensing activity................. 61

$2.5 \quad$ Discussion and conclusions.......................................... 62

$2.6 \quad$ Acknowledgements................................................ 66

Chapter 3: Inhibition of quorum sensing-controlled virulence factor production in Pseudomonas aeruginosa by south Florida plant extracts.......... 67

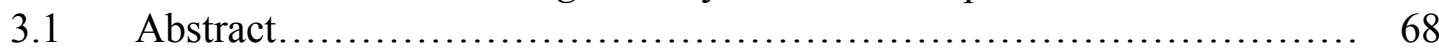

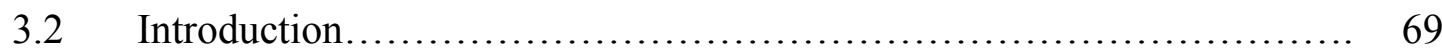

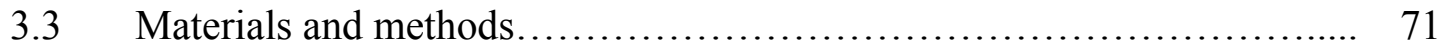

3.3.1 Plant extraction........................................... 71

3.3.2 Strains and media........................................... 72

3.3.3 Culturing conditions..................................... 72

3.3.4 LasA staphylolytic assay.................................. 72

3.3.5 LasB elastolytic assay.................................... 73

3.3.6 Pyoverdin assay.......................................... 73

3.3.7 Polyvinyl chloride (PVC) biofilm formation assay................ 74

3.3.8 AHL assay ................................................ 74

3.3.9 Growth curves............................................. 74

3.3.10 $\quad \beta$-Galactosidase assay..................................... 75

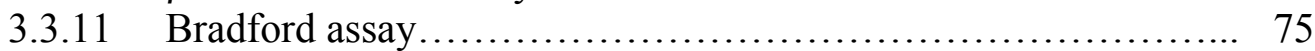

3.3.12 Statistical analysis......................................... 76

$3.4 \quad$ Results........................................................... 76

3.4.1 LasA protease activity in the presence of plant extracts.......... 76

3.4.2 LasB elastase activity in the presence of plant extracts.......... 76

3.4.3 Extracts alter pyoverdin production........................ 78

3.4.4 Plant extracts have an inhibitory effect on biofilm formation... 78

3.4.5 Plant extracts affect QS gene expression...................... 79

3.4.6 Plant extracts affect the production of AHL molecules........... 79

3.4.7 Extracts have minimal effect on PAO1 growth after log phase.. $\quad 80$

$3.5 \quad$ Discussion...................................................... 82

3.5.1 Plant extracts differentially affect biofilm formation............ 83

3.5.2 Plant extracts differentially affect production of virulence factors..................................................... 83

3.5.3 Mechanistic musings: multiple targets or global effect.......... 84

3.6 Conclusions......................................................... 86

$3.7 \quad$ Acknowledgments.................................................. 88 
Chapter 4: Attenuation of Pseudomonas aeruginosa virulence by medicinal plants in a

Caenorhabditis elegans model system .......................... 89

$4.1 \quad$ Abstract.......................................................... 90

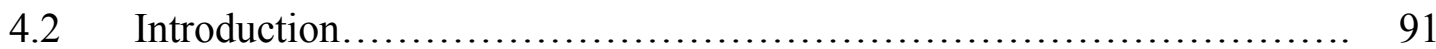

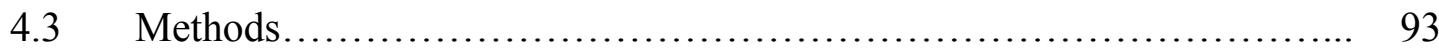

4.3.1 Preparation of plant extracts............................. 93

4.3.2 Bacterial strains and growth conditions...................... 93

4.3.3 Nematode culture........................................ 93

4.3.4 C. elegans paralytic assay (PA01)......................... 94

4.3.5 C. elegans fast killing assay (PA14)....................... 94

4.3.6 C. elegans slow killing assay (PA14)...................... 95

4.3.7 Statistics and reproducibility............................. 95

$4.4 \quad$ Results and discussion........................................... 95

4.4.1 Medicinal plants reduce C. elegans paralytic killing by

$P$. aeruginosa......................................... 96

4.4.2 Medicinal plants reduce C. elegans fast killing by P. aeruginosa............................................ 98

4.4.3 Medicinal plants reduce C. elegans slow killing by $P$. aeruginosa ............................................... 99

4.5 Conclusions.................................................. 100

$4.6 \quad$ Acknowledgements............................................ 101

Chapter 5: Ellagitannins from Conocarpus erectus exhibit anti-quorum sensing activity against Pseudomonas aeruginosa..................... 102

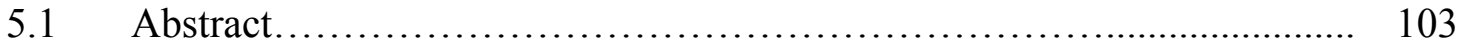

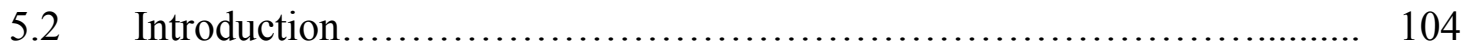

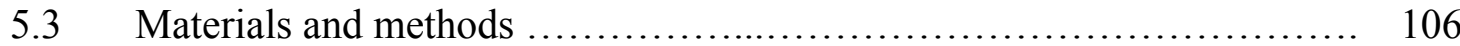

5.3.1. Isolation and verification of active compounds ............... 106

5.3.1.1 Plant extraction............................. 106

5.3.1.2 Bioassay-guided fractionation................. 106

5.3.1.3 Preliminary thin layer chromatography........... 107

5.3.1.4 Fractionation methods............................ 107

5.3.1.5 HPLC separation.............................. 108

5.3.1.6 Mass spectrometric analysis................... 108

5.3.1.7 NMR spectroscopy............................. 109

5.3.2 Biological assay.......................................... 109

5.3 .3 Statistical analysis............................................... 110

$5.4 \quad$ Results and discussion............................................. 110

5.4.1 Isolation and verification of active compounds............... 110

5.4.1.1 TLC reveals a phenolic compound responsible for anti-QS activity.......................... 110

5.4.1.2 Fractionation of polyphenolics in crude extract ... 112

5.4.1.3 HPLC separation reveals two fractions with anti-QS activity.............................. 114 
5.4.1.4 Vescalagin and castalagin elucidated as active compounds............................. 115

5.4.2 Bioassays on P. aeruginosa confirm anti-QS activity of ellagitannins.

5.4.2.1 LasA protease activity is reduced in the presence 117

5.4.2.2 LasB elastase activity is reduced in the presence

5.4.2.3 Ellagitannins do not affect pyoverdin production.

5.4.2.4 Ellagitannins affect the production of QS signaling molecules.

5.4 .2 .5

Ellagitannins affect QS gene expression.

120

5.4.2.6

Ellagitannins do not affect bacterial growth.

121

122

123

5.5 Concluding remarks

124

Chapter 6: General Discussion and Summary ............................... 126

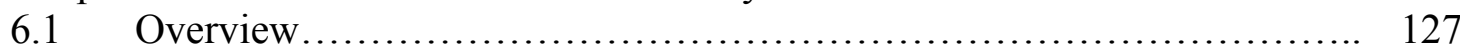

6.2 Anti-QS activity of medicinal plants............................... 128

6.3 Attenuation of Pseudomonas aeruginosa virulence......................... 130

6.4 Inhibition of $P$. aeruginosa virulence in a nematode model.................. 133

6.5 Ellagitannins are responsible for anti-QS activity ....................... 134

$6.6 \quad$ Future Directions.................................................... 135

BIBLIOGRAPHY ......................................................... 137

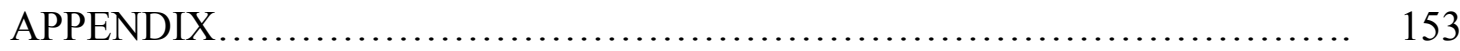

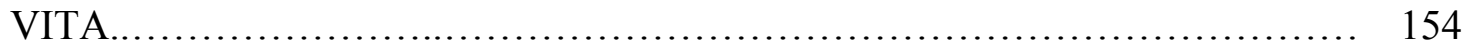




\section{LIST OF TABLES}

TABLE

PAGE

Table 2.1 Southern Florida medicinal plants tested for anti-QS activity...... 50

Table 2.2 Species showing anti-QS activity in this study................ 60

Table $3.1 \quad$ Effect of plant aqueous extracts on $P$. aeruginosa virulence

factors............................................ 77

Table 3.2 Effect of plant extracts on P.aeruginosa QS genes and

AHL production................................................ 80

Table 5.1 Effect of crude extract of $C$. erectus and purified ellagitannins

on $P$. aeruginosa virulence factors......................... 119

Table 5.2 Effect of ellagitannins on P. aeruginosa QS genes and AHL production............................................ 121 


\section{LIST OF FIGURES}

FIGURE

PAGE

Figure 1.1 The tribal and geographical segregations of the Glades peoples in Florida.

Figure 1.2 $\alpha$-Terpinol, a major monoterpenoid component of

M. alternifolia............................................ 10

Figure 1.3 Two phenolic terpenoids of $O$. vulgare...................... 11

Figure $1.4 \quad 8$-methoxyfuranodiene, a furanosesquiterpene of Commiphora species.................................. 12

Figure 1.5 Some of the various structures of AHLs, the main signaling molecule of Gram negative bacteria........................ 15

Figure 1.6 The LuxI/R paradigm................................. 16

Figure 1.7 Comparison of AHL and halogenated furanone................ 17

Figure 1.8 Some potential targets of anti-QS compounds................ 20

Figure 1.9 Multiple views of Pseudomonas aeruginosa................... 22

Figure $1.10 \quad$ Various sites of $P$. aeruginosa infection...................... 24

Figure 1.11 Common anti-pseudomonal antibiotics and mechanisms of action.................................................. 28

Figure $1.12 \quad$ "Gene transfer" ..................................... 30

Figure $1.13 \quad$ Biofilm formation..................................... 32

Figure 1.14 MexAB-OprM efflux pump of P. aeruginosa .................... 34

Figure 1.15 Core structure of Penicillin, typical of $\beta$-lactam antibiotics....... 35

Figure 1.16 The main QS system in P. aeruginosa ..................... 40

Figure 1.17 Caenorhabditis elegans................................... 41

Figure 2.1 Preliminary screening of C. erectus for anti-QS activity using C. violaceum biomonitor strain............................ 57 
Figure 2.2 Anti-QS activity using (a) C. violaceum and (b) A. tumefaciens strains.......................................................... 59

Figure 2.3 Antibacterial activity vs. anti-QS activity...................... 61

Figure 3.1 Influence of medicinal plant extracts on growth of P. aeruginosa............................................... 81

Figure 4.1 Nematode killing curves comparing wt, QS mutant, and wt with plant extract. ................................... 96

Figure 5.1 Thin layer chromatography (TLC) of C. erectus crude extract and the visualization of phenolic anti-QS activity............... 111

Figure 5.2 Schematic of fractionation of C. erectus crude extract............. 113

Figure 5.3 Anti-QS bioassay of fractionation products of C. erectus.......... 114

Figure 5.4 HPLC separation of Fraction A.............................. 115

Figure 5.5 Structures of the stereoisomeric ellagitannins castalagin

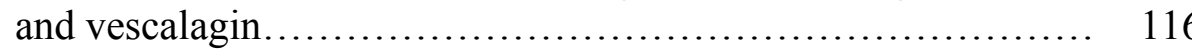

Figure 6.1 QS pathway of P. aeruginosa and possible activity of crude extracts and purified vescalagin 


\section{LIST OF ABBREVIATIONS}

TERM / UNIT OF MEASUREMENT

5-bromo-4-chloro-3-indolyl- $\beta$-D-galactopyranoside

Acetonitrile

Acylhomoserine lactone

Agrobacterium (minimum) media

Analysis of variance

Brain-heart infusion

Correlation spectroscopy

Cystic fibrosis

Cystic fibrosis transmembrane conductance regulator

Degree Celsius

Deuterated trifluoroacetic acid

Deuterium oxide

Electrospray ionization

et alia

Ethanol

Extended-spectrum of $\beta$-lactamase

Gram

Heteronuclear multiple bond correlation

Heteronuclear multiple quantum coherence

High pressure liquid chromatography

Hour

Kilovolt
SYMBOL / ABBREVIATION

X-gal

$\mathrm{ACN}$

AHL

$\mathrm{AB}$

ANOVA

BHI

COSY

CF

CFTR

${ }^{\circ} \mathrm{C}$

dTFA

$\mathrm{D}_{2} \mathrm{O}$

ESI

et al.

$\mathrm{EtOH}$

ESBL

g

HMBC

HMQC

HPLC

h

$\mathrm{kV}$ 
Liquid chromatography LC

$\begin{array}{ll}\text { Luria-Bertani } & \text { LB }\end{array}$

Mass spectrometry $\quad$ MS

Mass-to-charge ratio $\mathrm{m} / \mathrm{z}$

Megahertz $\quad \mathrm{MHz}$

Microgram $\quad \mu g$

Microliter $\quad \mu 1$

Micrometer $\mu \mathrm{m}$

Micron $\mu$

Milligram $\quad \mathrm{mg}$

Milliliter $\mathrm{ml}$

Millimeter $\mathrm{mm}$

Millimolar $\quad \mathrm{mM}$

Minimal inhibitory concentration $\quad$ MIC

Minutes $\min$

N-(3-oxododecanoyl)-L-homoserine lactone OdDHL

Nanometer $\quad \mathrm{nm}$

N-butanoyl-L-homoserine lactone BHL

Nematode growth media $\quad$ NGM

$\begin{array}{ll}\text { Nuclear magnetic resonance } & \text { NMR }\end{array}$

Optical density $\quad$ OD

Ortho-nitrophenyl- $\beta$-D-galactopyranoside ONPG

Penicillin-binding protein $\quad$ PBP

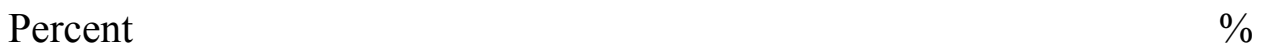


Pounds per square inch psi

Quorum sensing QS

Thin layer chromatography $\quad$ TLC

Ultraviolet UV

Water $\quad \mathrm{H}_{2} \mathrm{O}$

Wild type WT 


\section{Chapter 1}

Introduction 


\subsection{Preface}

The following introduction includes a review of the literature and provides the reader with a background for the experimentation to follow. As this is a multidisciplinary project, a number of different fields and sub-fields are explored with relevance to this dissertation, including ethnobotany, medicinal phytochemistry, and quorum sensing as a target to attenuate bacterial virulence and disease. Attention is also given to the nature of the model pathogen Pseudomonas aeruginosa, its mechanisms of antibiotic resistance, and its behavior in an nematode infection model.

\subsection{Ethnobotany and pharmacognosy: looking to the past for solutions of the future}

Before pharmacy there was forest, before pills there were potions. The emergence of medicine paralleled that of the human species, and as we evolved so did our pharmacopoeia. Modernization of our society and concomitant advances in chemistry promised better, stronger, safer, and more widely available drugs. However the overuse of antibiotics began an escalating trend of resistance in many pathogenic organisms. In effect, our diseases were evolving right along with us.

Although combinatorial and synthetic chemistry can provide us with some solutions for disease, many medicinal compounds already exist in nature and are awaiting discovery. Vascular plants are one of the largest and most diverse organismal groups with tropical and sub-tropical regions affording a high species biodiversity (Gurevitch et al., 2002). This high biological diversity is likely linked to a high chemical diversity, allowing for the evolution of numerous toxic and bioactive plant chemicals. 
Phytochemistry, however, is directed by more than sheer randomness. Plants create specific compounds to serve needs such as reproduction and defense. Limited by their immobility, plants must rely on wind, water, or animals for pollination. The availability and precision of the former being somewhat uncertain, plants have adapted various chemical means of attracting animals. Anthocyanin-based pigments attract birds, sweet smelling phenolic volatiles attract bees, and foul indoles and amines are perfect for fly pollination (Gurevitch et al., 2002).

Plants have likely evolved toxic and bioactive compounds to protect against herbivory and pathogen attack. (Balick \& Cox, 1999; Gurevitch et al., 2002). Because plants are sessle, they rely more heavily on chemical defenses than motile organisms. In many cases, the link between a toxin and a medicine is dosage, and numerous plant toxins have found their way into our modern pharmacopoeia. Foxglove, Digitalis spp., if ingested, can cause convulsions, cerebral disturbances, bradycardia, and eventual death (Lacassie et al., 2000). However, at the correct dosage, the cardiac glycosides, digoxin and digitoxin, have proven effective in the treatment of atrial fibrillation and heart failure (Bussey et al., 1988).

\subsubsection{Advantages of a directed search}

Taking into account the vast number of plants that exist, and their evolved chemical diversity, one can see the basis for screening plants for medicinal compounds. However, combining these inherent qualities of plants with the direction of thousands of years of amassed traditional knowledge creates an even better venue in which to search for new drugs. 
Most plants contain some sort of constitutive or inducible defense against phytopathogens, but plants used medicinally may also contain chemical defenses specific to human pathogens. In fact, the efficiency of an ethnobotanically-directed search to this end has proven to be about $40 \%$ higher than that of a random search (Khafagi \& Dewedar, 2000).

The word ethnobotany, from the Greek ethnos 'people', is defined as 'the study of the interactions between people and plants" (Harshberger, 1896). It is a scholarly pursuit somewhere between science and anthropology devoted to documentation and understanding of the traditional classification and uses of plants in different human societies. Studies in ethnobotany have provided us with lists of potentially medicinal plants, but rarely a knowledge of their chemical nature.

Pharmacognosy was a term introduced by C.A. Seydler, a German medical student, in 1815, derived from the Greek pharmakon, or drug, and gnosis, knowledge (Tyler et al., 1981). The field of pharmacognosy was borne of pharmacology due to the need for a more comprehensive approach to drug discovery and understanding. Swiss pharmacist Friedrich Flueckiger expounded upon this idea stating that pharmacognosy is "the simultaneous application of various scientific disciplines with the object of acquiring knowledge of drugs from every point of view" (Tyler et al., 1981) including botany, microbiology, taxonomy, phytochemistry, medicinal chemistry, and toxicology. In this sense it is not so much a "subfield" of pharmacology as it is a larger, more comprehensive idea entirely. Today, the term pharmacognosy typically refers to the pharmacology of natural products (As defined by the American Society for Pharmagognosy www.phcog.org). The combination of ethnobotany and pharmacognosy 
opens up an avenue of research into the potential drug applications of traditionally used plants, often referred to as ethnopharmacology or ethnopharmacognosy.

\subsubsection{Linking traditional and modern medicine}

Ethnopharmacology puts drug discovery in a cultural construct. Making the link back to a cultural use is beneficial for a number of reasons, both to traditional and modern societies. Knowing the traditional use of a plant can guide drug discovery, by giving chemists and biologists an idea of its potential use in modern society. For instance, plants used as snakebite remedies may be useful in finding antihypertensive drugs. If one wants to survive the bite of a venomous snake, it is advantageous to lower the blood pressure and slow the heart rate so as to not spread the poison to vulnerable organs before it can be metabolized. This led to the discovery of antihypertensive alkaloids in Rauvolfia spp. (often R. serpentina (L.) Benth. ex. Kurz), a small shrub in the Apocynaceae (Dewick, 2002).

However, ethnopharmacology is not so myopic as to focus solely on the profit of developed countries. Much of the work done in this field benefits traditional societies. The documentation and preservation of indigenous pharmacopeias and healing techniques, is becoming increasingly important with modernization and migration of cultures. In addition, validation through modern scientific methods may help improve healthcare in rural areas. The ideal ethnopharmacological study would begin with the cultural anthropology of a particular group and their medical system in the context of that culture, and include the documentation of indigenous medical knowledge to prevent loss of information in future generations. There would have to be a botanical, chemical, and 
pharmacological workup of the plants to potentially derive useful and unique medicinal compounds. Each of these areas (anthropology, botany/taxonomy, chemistry, pharmacology) could take years to fully explore. Consequently, most studies focus on one aspect of ethnopharmacology, depending on the expertise and/or interests of the researcher.

\subsubsection{The ecology and ethnobotany of southern Florida}

Southern Florida has a unique diversity of species due to its location between tropical and temperate climates. This subtropical ecosystem is host to over 1600 native and naturalized plants from various regions (Wunderlin, 1982; Wunderlin \& Hansen, 2006). The diverse ecotypes of Miami-Dade County include coastal environments, scrub and tropical dry forests, and pine rocklands, each with its own distinct cohort of species.

Plants in this region have provided important sources of food, timber, and medicines for Native Americans and early European settlers. Despite the fact that they lived along the coasts, the original inhabitants of modern-day Florida were dubbed the "Glades Indians" by archaeologists (Goggin \& Sturtevant, 1964). They segregated into a number of distinct tribes including the Calusa, Tequesta, Mayaimi, and Jaega tribes of southern Florida (McGoun, 1993) (Figure 1.1).

In the $17^{\text {th }}$ and $18^{\text {th }}$ centuries, the indigenous people of south Florida increased not only their plant knowledge, but presumably their physical pharmacopoeia by trading with nearby Cuban, Mexican, and Caribbean cultures. Advances in transportation led to increased dissemination and accumulation of both knowledge and plant material. Plant uses and the plants themselves moved into the area from lands as far as Central and South 
America and Africa (Austin, 1997). However, by the mid-18th century, European slavery and disease had decimated the once powerful Glades peoples, and by 1763 all of Florida's pre-Columbian cultures were extinct (Milanich, 1995).

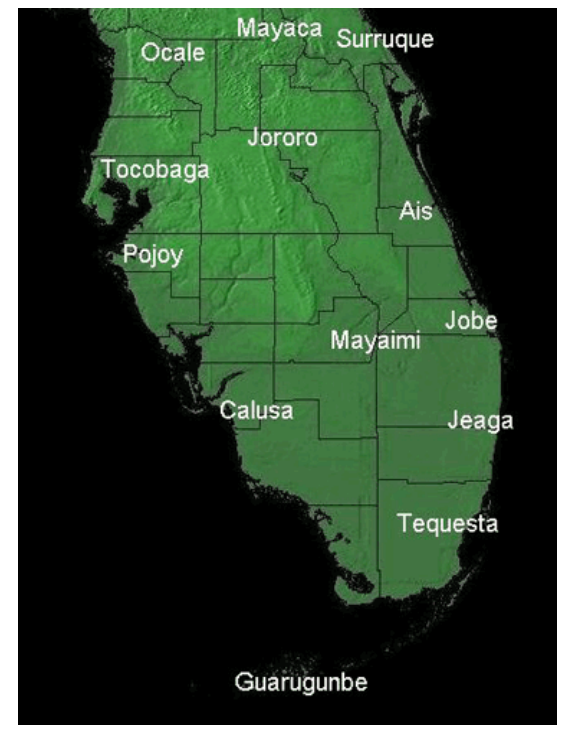

Figure 1.1 The tribal and geographical segregations of the Glades peoples in Florida. Florida Museum of Natural History. Used with permission.

In the $17^{\text {th }}$ and $18^{\text {th }}$ centuries, the indigenous people of south Florida increased not only their plant knowledge, but presumably their physical pharmacopoeia by trading with nearby Cuban, Mexican, and Caribbean cultures. Advances in transportation led to increased dissemination and accumulation of both knowledge and plant material. Plant uses and the plants themselves moved into the area from lands as far as Central and South America and Africa (Austin, 1997). However, by the mid-18th century, European slavery and disease had decimated the once powerful Glades peoples, and by 1763 all of Florida's pre-Columbian cultures were extinct (Milanich, 1995). 
Although most estimates suggest roughly $20 \%$ of any native flora to be used by its inhabitants, sadly only about $3 \%$ of the flora of southern Florida has been documented as initially utilized by the Glades peoples, and approximately $10 \%$ by later inhabitants of the region (Austin, 1997; Austin, 2004). However more data about medicinal use can be pieced together from the ethnobotany of other cultures within the growth habitat of the plant. For instance, while historical usage of buttonwood, Conocarpus erectus (Combretaceae) is mainly confined to timber or fuel use in Florida; cultures from Mexico, South America, the Caribbean, and Africa use this tree as a tonic, febrifuge, and to treat various bacterial conditions (Austin, 1997; Burkhill, 1985; Melendez, 1982; Morton, 1981).

Under the guidance of traditional knowledge, many plants have been discovered to be useful in our modern system of medicine. Notable medicinal plants of southern Florida include saw palmetto and the Madagascar periwinkle. Saw palmetto, Seranoa repens (Aracaceae), is perhaps the most well-known plant from this region used in the treatment of benign prostatic hyperplasia (Bennett et al., 2002; Madersbacher et al., 2007). Whereas, the naturalized periwinkle Catharanthus roseus (Apocynaceae), produces vinca-alkaloids used in the treatment of cancer (Heijden et al., 2004).

Although the plants chosen for this study are not a cohesive group of native species used by one particular indigenous culture, they have a long history of medicinal use throughout their native and naturalized range (Burkhill, 1985; Duke, 1972; Duke, 1985; Duke, 2000; Liogier, 1990; Morton, 1981). Additionally, all plants chosen have been used historically to treat conditions such as wounds, infections, respiratory conditions, and diarrhea - conditions potentially caused or complicated by bacteria. 


\subsection{Botanicals as anti-bacterial therapies}

There have been many ethnobotanically-directed searches for antibacterials, demonstrating not only the need for these drugs, but also the large number of plant species utilized for bacterial conditions especially in the tropics (Camporese et al., 2003; Cowan, 1999; Gnanamani et al., 2003; Hernandez et al., 2003). Although medicinal plant research has led to the discovery of many important medicines such as morphine (Benyhe, 1994), quinine (Kremsner et al., 1994), paclitaxel (Wani et al., 1971), and camptothecin (Wall et al., 1966), there is not a large degree of overlap between commonly used plant medicines and antimicrobials in developed countries. However, there are a few notable plants that have made their way to the drugstore shelves.

\subsubsection{Tea tree oil}

Melaleuca alternifolia Cheel (Myrtaceae), or tea tree, is a aromatic tree native to Australia. The essential oil of M. alternifolia is a common topical antimicrobial agent. Its antimicrobial activity has been attributed to terpinen-4-ol, the major monoterpenoid component of the oil (Figure 1.2) (Carson \& Riley, 1995). Other terpene constituents include $\gamma$-terpinene, $\alpha$-terpineol, cineole, and $p$-cymene (Dewick, 2002). Monoterpenoids are composed of two isoprene units and occur widely in plants with volatile oils such as those in the Lamiaceae, Myrtaceae, and Rutaceae. These compounds tend to cause membrane disruption in bacteria (Cowan, 1999), and in addition account for much of the aroma of a plant. 


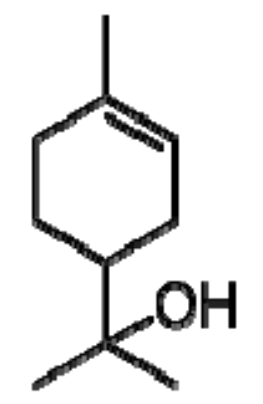

Figure $1.2 \alpha$-Terpinol, a major monoterpene component of $M$. alternifolia (http://en.wikipedia.org/wiki/Image:Terpineol_alpha.svg)

Tea tree oil has been proven in several in vitro (Hammer et al., 1996; Messager et al., 2005), and topical studies (Carson et al., 1998; Satchell et al., 2002) to be antibacterial and antifungal. It is effective against Staphylococcus aureus, Acinetobacter baumannii, Escherichia coli and Pseudomonas aeruginosa, at a 5\% concentration in hand wash (Messager et al., 2005). It is also active against multi-drug resistant strains of $S$. aureus (Halcon \& Milkus, 2004; Park et al., 2007).

\subsubsection{Oregano oil}

Origanum vulgare L. (Lamiaceae) is a common herb native to Europe and the Mediterranean. The essential oil fraction of $O$. vulgare is especially effective against bacterial and fungal infections, of the gastrointestinal and genitourinary tract (Adam et al., 1998; Blumenthal et al., 2000; Chun et al., 2005; Salgueiro et al., 2003). Its antimicrobial activity is attributed to the phenolic monoterpenoids, carvacrol and thymol, along with a number of other terpene alcohols, phenols, and sesquiterpenes (Rodrigues $e t$ 
al., 2004). The two main chemotypes of $O$. vulgare show either high thymol or high carvacrol content (Figure 1.3) (Rodrigues et al., 2004). Both thymol and carvacrol are antimicrobial (Cowan, 1999), however the latter is considered more effective (Chami et al., 2004).
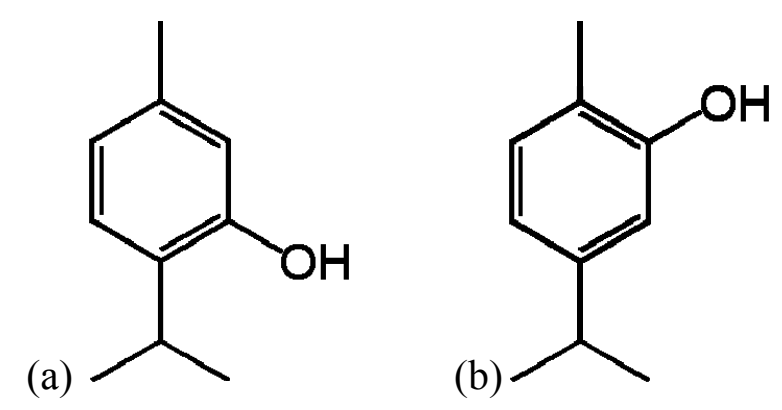

Figure 1.3 Two phenolic terpenoids of O. vulgare. (a) Thymol. (b) Carvacrol. (http://en.wikipedia.org/wiki/Image:Thymol.png and http://en.wikipedia.org/wiki/Image: Carvracol.png )

Oil of oregano has pronounced antifungal effect in vitro (Adam et al., 1998) against a number of dermatophytes. It also shows antibacterial activity against Helicobacter pylori, a causative agent of gastric ulcers (Chun et al., 2005), and a number of clinically-isolated pathogens including Haemophyllus influenzae, Staphylococcus aureus, Escherichia coli, Streptococcus pneumoniae, and Enterobacter cloacae (HerschMartinez et al., 2005). Though most related to its prescribed use is its marked effect in vitro and in a rat model against C. albicans (Chami et al., 2004; Salgueiro et al., 2003). The antimicrobial effect is due to membrane disruption (Chami et al., 2004; Cowan, 1999). 


\subsubsection{Myrrh}

Myrrh is a fragrant, oleo-gum resin of various Commiphora Jacq. species in the Buseraceae, a family consisting mainly of trees with aromatic, peeling bark (Judd et al., 2002). Commiphora trees are native to parts of Africa, Asia, India, and the Middle East, where the bark is scored to collect the resin (Blumenthal et al., 2000). Myrrh has a long history of religious and medicinal use with references in Jewish, Muslim, and Christian texts (Blumenthal et al., 2000; Cowan, 1999). It still sees use today in religious settings, but is more commonly found in mouthwashes and toothpastes due to its antimicrobial properties.

Myrrh consists of approximately 30-60\% water soluble gum, 20-40\% alcohol soluble resin, and 8\% volatile oil (Blumenthal et al., 2000). The volatile oil fraction contains antimicrobial monoterpenes such as myrcene and $\alpha$-camphorine, however antimicrobial activity is also attributed to furanosesesquiterpenes (Figure 1.4) (Blumenthal et al., 2000).

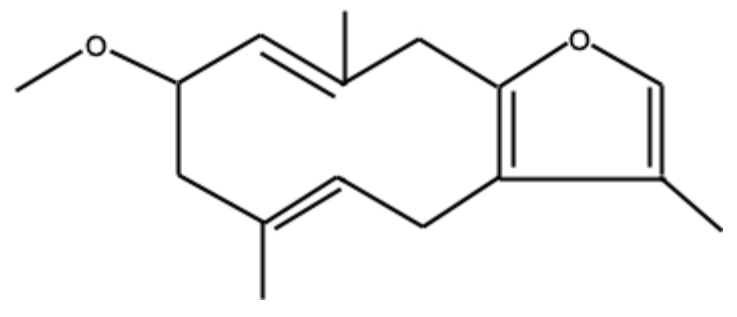

Figure 1.4 8-methoxyfuranodiene, a furanosesquiterpene of Commiphora species. (Redrawn from http://chembiofinder.cambridgesoft.com) 
The antibacterial activity of Commiphora mulkul (synonym of Commiphora wightii (Arn.) Bhandari) has been tested in vitro against a number of Gram positive and Gram negative organisms and was found equal to that of kanamycin (Asif Saeed \& Sabir, 2004). The German commission E recognizes myrrh as an antibacterial and antiinflammatory. It is approved for topical treatment of wounds, and for oral and pharayngeal mucosa as a mouthwash (Blumenthal et al., 2000).

\subsubsection{Untapped potential for antimicrobial activity}

As mentioned previously, plants should be a great source of antimicrobial compounds due to their environment. However, in comparison with other organisms, plants do not have the monopoly on medicinal compounds especially in the case of antimicrobials. In fact, $75 \%$ of the modern antibiotics are derived from the actinomycetes, a specific group of Gram-positive bacteria (Mendez \& Salas, 2001). Regardless, there are a few reasons why this should not deter one from screening plants for antimicrobial compounds.

Antibiotic-producing microbes possess genes which protect them from the toxic effects of these compounds. It is very easy for one microbe to acquire antibiotic resistance from another through plasmid transfer or transposons. Plants, on the other hand, are genetically dissimilar from the organisms they are trying to eradicate. Thus, there is little chance for a microbe to gain resistance from a plant.

Plants can produce a multitude of diverse antimicrobial compounds such as simple phenolics, catechins, quinones, flavanones, polyphenolics, alkaloids, and terpenoids (Cowan, 1999; Dewick, 2002). Like microbial antibiotics, these compounds 
are targeted at killing the pathogen and work via a non-species specific mechanism such as disrupting microbial cell membranes (Cowan, 1999). However, it was recently discovered that plants have another way of dealing with microbes - targeting a cell's communication system (Bauer, 2001). One form of intercellular communication in bacteria has been studied since the 1970's, and is known as quorum sensing (for a review see (Schauder \& Bassler, 2001). Breakdown of this system causes an attenuation of microbial pathogenicity (Finch et al., 1998; Smith \& Iglewski, 2003b; Wu et al., 2004). The discovery of anti-quorum sensing agents in plants provides us with yet another type of "antimicrobial" agent.

\subsection{Quorum sensing: a novel target}

Quorum sensing (QS) is a population-dependent phenomenon first characterized in the 1970s in luminescent marine species of Vibrio (Nealson et al., 1970). The ability to sense the size of a bacterial population is mediated through small signaling molecules or autoinducers (Hastings \& Greenberg, 1999; Schauder \& Bassler, 2001). These molecules are constantly produced and received at a basal level by bacterial cells. With high population density, there is a surplus of signaling molecules in the environment. These signals diffuse back into the cell where they facilitate the regulation of gene expression (Hastings \& Greenberg, 1999). QS systems are ubiquitous among bacteria, and have since been found to regulate diverse functions such as luminescence, biofilm formation, antibiotic and virulence factor generation, pigment production, plant-microbe interactions, and motility (Hentzer et al., 2003; McClean et al., 1997; Rasmussen et al., 2000; Rodelas et al., 1999; Shih \& Huang, 2002). 
Although there are a number of different QS systems (Henke \& Bassler, 2004), the most widely studied paradigm is based on the Lux system of Vibrio fisheri and $V$. harveyi (Bassler et al., 1993; Stevens \& Greenberg, 1997). This QS phenomenon involves a three component-system: a freely diffusible signal, a synthetase to make this signal, and a regulator that interacts in conjunction with the signal to regulate gene expression. The main signaling molecules produced by Gram-negative bacteria are acylhomoserine lactones (AHLs) (Fuqua \& Greenberg, 1998). They differ in the length of their side chains and substitution at the $\mathrm{C} 3$ carbon, based on the organism that produces them (Marketon et al., 2002; Whitehead et al., 2001a). (Figure 1.5)
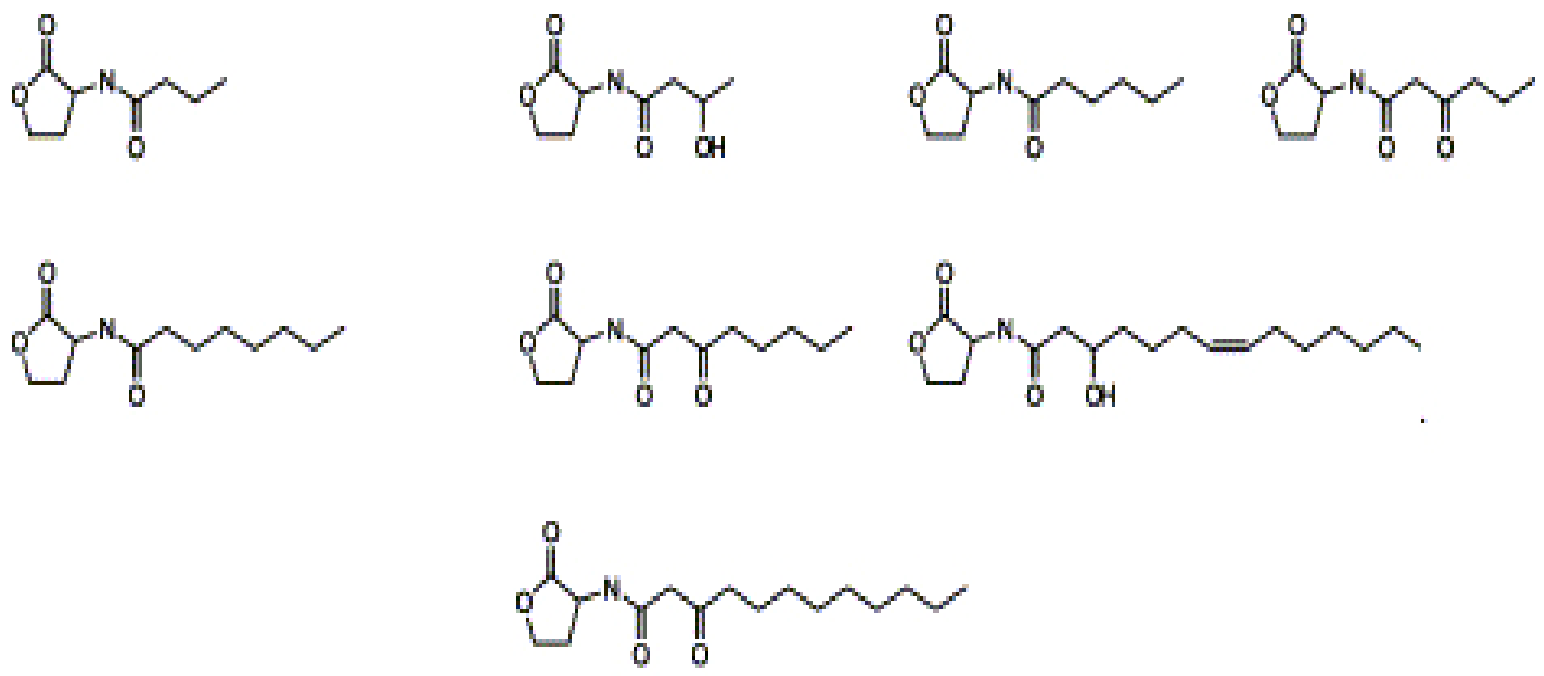

Figure 1.5 Some of the various structures of AHLs, the main signaling molecule of Gram negative bacteria. Length of the hydrocarbon side chain can range from $\mathrm{C} 4$ to $\mathrm{C} 18$, and the $\mathrm{C} 3$ carbon may be substituted with an oxygen or hydroxyl group (modified from (Whitehead et al., 2001a). 
In V. fisheri, LuxI produces an AHL signaling molecule which binds to LuxR at a certain concentration (Stevens \& Greenberg, 1997). This concentration is reached only when enough bacteria are present (a quorum) to produce sufficient quantities of the AHL. The $V$. fischeri LuxR-AHL complex can then bind to the operator and upregulate transcription of the luciferase genes. (Figure 1.6)

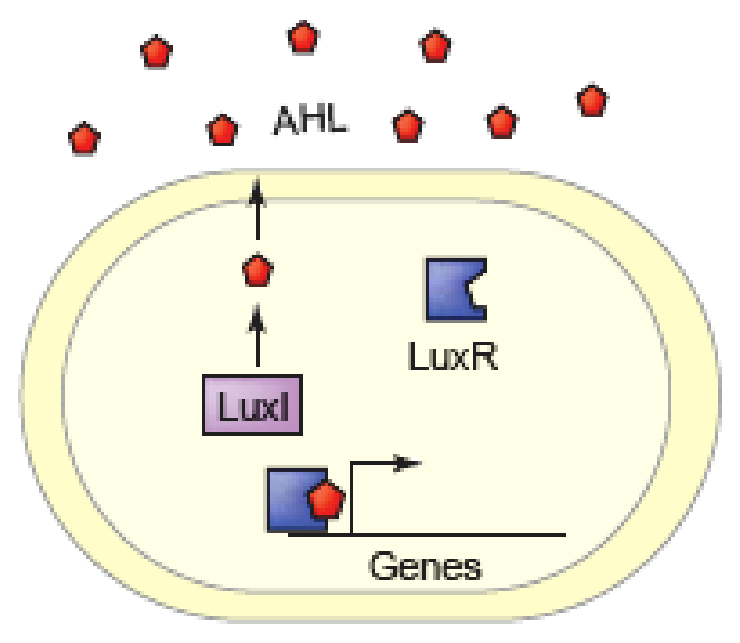

Figure 1.6 The LuxI/R paradigm. LuxI is produced at a basal level in the cell which synthesizes AHLs (red pentagons). The AHLs bind to LuxR (product of luxR) at a certain concentration and activate transcription of $l u x I$ and the luciferase genes (Henke \& Bassler, 2004). Used with permission.

AHL-mediated quorum sensing systems based on the LuxI/R paradigm have been characterized in human pathogens such as Pseudomonas aeruginosa (Pesci et al., 1997), Yersinia pseudotuberculosis (Atkinson et al., 1999), and Escherichia coli (Surette \& Bassler, 1998), as well as plant associated bacteria such as Rhizobium leguminosarum 
(Rodelas et al., 1999), Ralstonia solanacearum, and Erwinia carotovora (Von Bodman et al., 2003). In all cases these systems can regulate virulence. Thus, the discovery of QS has given us a new target - a new way to attack and attenuate bacterial pathogenicity.

\subsubsection{Anti-quorum sensing}

The subsequent discovery of compounds that inhibit cell-to-cell communication, dubbed anti-quorum sensing (anti-QS) agents could provide a novel method of combating infection (Hentzer \& Givskov, 2003a; Smith \& Iglewski, 2003c).

Anti-QS agents were first characterized in the red marine alga, Delisea pulchura (Manefield et al., 1999). This alga was investigated for its anti-fouling properties, and was found to contain halogenated furanones, compounds which block AHLs via competitive inhibition and destabilization of LuxR (Figure 1.7) (Manefield et al., 2002).

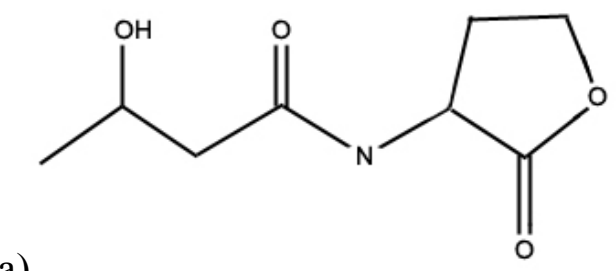

(b)

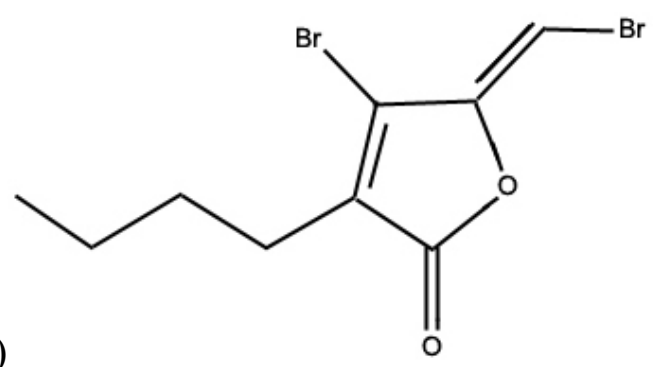

Figure 1.7 Comparison of AHL (a) and halogenated furanone (b). The structural similarity allows furanones to competitively inhibit the action of AHL signaling molecules. (AHL redrawn from http://chembiofinder.cambridgesoft.com, furanone redrawn from (Manefield et al., 2002)) 
Delisea furanones have been shown to reduce light emission in Vibrio species (Givskov et al., 1996), inhibit pigment production in C. violaceum (Martinelli et al., 2004) and attenuate exoenzyme production and swarming motility in Serratia liquefaciens (Rasmussen et al., 2000). Both natural and synthetic derivatives of Delisea furanones have also been shown to attenuate $P$. aeruginosa infection in vitro and in a murine model (Hentzer et al., 2003; Wu et al., 2004).

Since the discovery of AHL inhibitors in D. pulchura, anti-QS activity has been found in a south Florida Caulerpa species (Willsie, 2000) and a number of higher plants including various fruits and vegetables (Rasmussen et al., 2005; Teplitski et al., 2000; Vattem et al., 2007).

Pisum sativum (pea) seedlings and root exudates produced an inhibition of pigment production, exochitinase activity, and protease activity in C. violaceum (Teplitski et al., 2000). Some fractionation of the crude extract was attempted, however, no active compounds were purified.

Garlic (Allium sativum), carrot (Daucus carota), chamomile (Matricaria sp.), water lily (Nymphaea sp.) and various peppers (Capsicum spp.) were found to possess anti-QS activity against a luxI-gfp reporter strain (Rasmussen et al., 2005). Garlic was also found to inhibit biofilm formation in $P$. aeruginosa, and prevented nematode death in a limited analysis (Rasmussen et al., 2005). Garlic extract was partially fractionated in this study, but again, no purified compounds were elucidated.

Various fruits and herbs were recently shown to possess anti-QS activity in a $C$. violaceum biomonitor strain and on the swarming motility of E. coli and P. aeruginosa (Vattem et al., 2007). Fruits including raspberry, blueberry, blackberry, cranberry, and 
grape, and herbs such as thyme, ginger, basil, kale, oregano and turmeric exhibited moderate inhibition of these QS-controlled processes. However, no data was obtained in a live animal model, nor were active compounds purified from the extracts. The latter two studies were published after the publication of the initial work in this dissertation, indicating an increasing trend in this area of research.

Other than signal mimics such as the furanones and synthetic derivatives, the compounds ellagic acid, tannic acid, and epigallocatechin gallate have been shown to inhibit QS in both an E. coli and a P. aeruginosa biomonitor strain (Huber et al., 2004). These and related polyphenolics are widespread throughout the plant kingdom and should be further explored as anti-QS compounds.

\subsubsection{Mechanisms of QS inhibition}

There are a number of ways to inhibit cell-cell communication (Figure 1.8) including competitive inhibition, signal binding, degradation of the signaling molecule, and inhibition of upstream precursors or genetic regulation systems.

Success has been seen with competitive inhibition in the case of the furanones, (Figure 1.8b) however, many other QS antagonists have since been discovered (Kline et al., 1999; Smith et al., 2003). These antagonists are based on the C12-AHL structure and cause a reduction in LasR activity.

AHL-antibodies have also been developed to suppress QS through signal binding (Figure 1.8c) (Debler et al., 2007; Smith \& Iglewski, 2003b). A C12-AHL-protein conjugate was able to sucessfully inhibit lasB expression (Smith and Iglewski 
unpublished data), and a similar molecule with extremely high binding affinity for $\mathrm{C} 12$ AHL was recently crystallized and characterized (Debler et al., 2007).

Blocking S-adenosyl methionine (SAM) or the fatty acid precursors necessary to synthesize AHLs leads to decreased production of C12-AHL by LasI (Figure 1.8e) (Hoang et al., 2002). Of course genetic modification of upstream global regulators such as Vfr and GacA has also been shown to greatly reduce QS activity and the subsequent production of virulence factors (Albus et al., 1997; Reimmann et al., 1997).

a

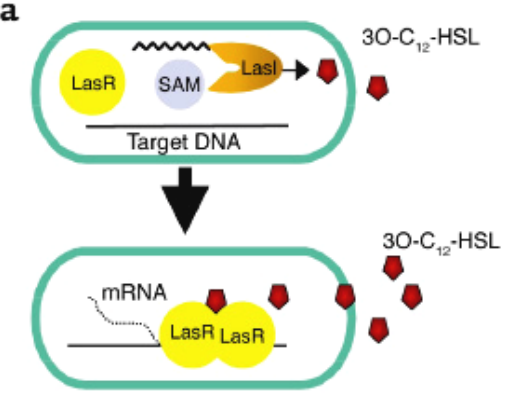

b

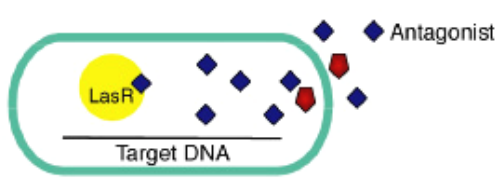

C

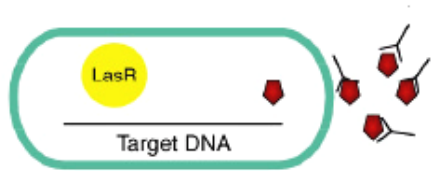

d

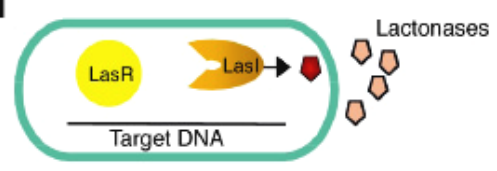

e

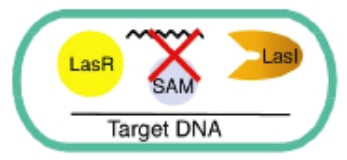

f

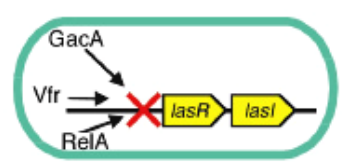

$\mathrm{g}$

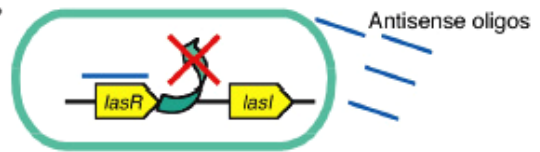

Figure 1.8 Some potential targets of anti-QS compounds (Smith \& Iglewski, 2003a). The $P$. aeruginosa $\mathrm{QS}$ system is shown in (a). Inhibition may occur via competitive inhibition by an AHL mimic (b), sequestration of the AHL signal (c), signal degradation by lactonases (d) or acylaces (not shown), inhibition of AHL synthesis (e), blocking of upstream regulation (f), and interference by antisense RNA (g).Used with permission. 
Scientists certainly do not have the monopoly on quorum inhibition. Numerous bacteria including Bacillus sp., Variovorax paradoxus, Arthrobacter sp., and A. tumefaciens produce lactonases - enzymes that cleave and deactivate the lactone ring of various AHLs (Carlier et al., 2003; Dong et al., 2002; Leadbetter \& Greenberg, 2000; Park et al., 2003) (Figure 1.8d). Lactonase expression in P. aeruginosa, results in a significant decrease in AHL production and virulence factor expression (Reimmann et al., 2002).

Interest is growing in practical applications of anti-QS especially when faced with increased incidence of drug failure due to the large number of pathogenic bacteria developing resistance to available antibiotics. It has been suggested that targeting pathogenesis instead of killing the organism may provide less selective pressure and therefore decreased emergence of resistant strains (Hentzer \& Givskov, 2003b; Whitehead et al., 2001b).

\subsection{Model organism: Pseudomonas aeruginosa}

Pseudomonas aeruginosa is a Gram-negative, aerobic bacillus, with length and width ranging from 1.5-3.0 $\mu \mathrm{m}$ and 0.5-0.8 $\mu \mathrm{m}$ respectively (Figure 1.9) (Bergey, 2001). It is motile by means of a single polar flagella, and is an oxidase-positive, nonsporulating and non-fermentative species (Bergey, 2001). Other diagnostic characteristics include beta-hemolysis of blood agar, pigment production - including pyocyanin (blue-

green), pyoverdin (yellow-green), and pyorubin (red-brown), and a distinct grape-like odor (Bergey, 2001). It is a ubiquitous organism with the ability to colonize diverse 
niches due to its range of metabolic capabilities and resistance to environmental challenges. (Vasil, 1986).

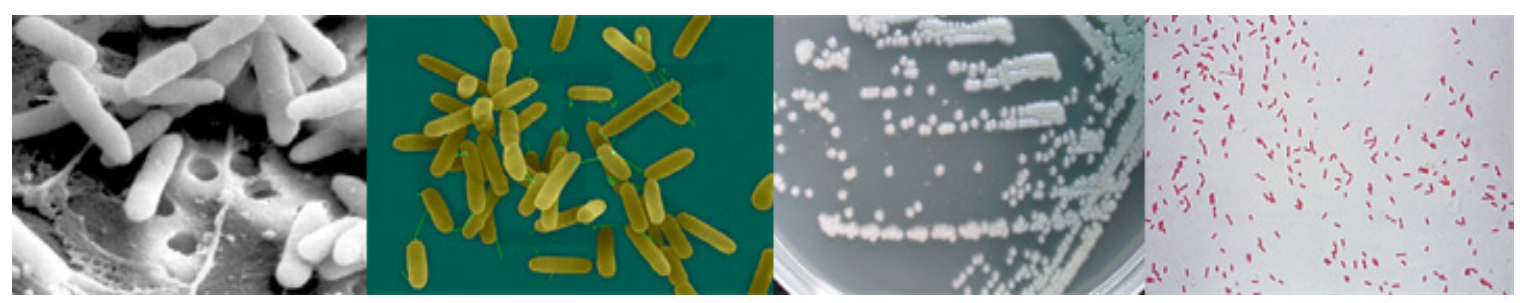

Figure 1.9 Multiple views of Pseudomonas aeruginosa. From L to R: Scanning electron microscopy, false-color microscopy, colonies on agar, gram stained cells. (http://upload.wikimedia.org/wikipedia/commons/thumb/c/cf/Pseudomonas_aeruginosa_ SEM.jpg;http://www.bact.wisc.edu/themicrobialworld/nfP.aeruginosa_colonies.jpg,http:/ /news.bbc.co.uk/olmedia/1745000/images/_1746739_pseudomonas_aeruginosa300.jpg http://www.pseudomonas.com/images/paeruginosa.jpg)

\subsubsection{Virulence factors and toxins}

Contributing to the pathogenicity of $P$. aeruginosa are a multitude of secreted toxins and virulence factors including, phospholipase $\mathrm{C}$, rhamnolipid, superoxide dismutase, $\mathrm{HCN}$, exotoxin A, exoenzyme, pyocyanin, pyoverdin, Las A protease and LasB elastase (Baker, 1982; Bever \& Iglewski, 1988; Coburn, 1992; Doring et al., 1987; Edwards \& Hayashi, 1965; Lau et al., 2004; Meyer et al., 1996; Stinson \& Hayden, 1979). Many of these factors cause or catalyze reactions in the host leading to cell death and tissue necrosis, however the latter three factors are focused on this study. 
Pyoverdin, a yellow-green florescent pigment produced by $P$. aeruginosa, competes with mammalian transferrin for iron, the successful sequestration of which essentially starves the host tissues (Meyer et al., 1996). It also promotes pathogenicity by stimulating bacterial growth (Cox \& Adams, 1985).

LasA and LasB are zinc metalloendopeptidases of $20-\mathrm{kDa}$ and $33-\mathrm{kDa}$ respectively, belonging to the $\beta$-lytic endopeptidase family of proteases (Toder et al., 1994). LasA has a limited substrate specificity as compared to LasB, however they operate in conjunction to degrade elastin, a key component in tissue plasticity. LasA nicks elastin allowing it to be cleaved by LasB and other proteases. Working together these proteases are capable of destroying or inactivating a wide range of biological tissues and immunological agents (Bever \& Iglewski, 1988; Kessler et al., 1993).

\subsubsection{Pseudomonas aeruginosa disease association}

Due to its arsenal of virulence factors and the tendency to form intractable biofilms (discussed later in this section), P. aeruginosa is not surprisingly one of the leading organisms implicated in nosocomial infections. According to a detailed hospital survey conducted from 1990-1996, P. aeruginosa was the second most prevalent cause of nosocomial pneumonia (17\% of isolated microbes), the third most common cause of urinary tract infections (11\%), the fifth most common cause of surgical site infections ( 8 $\%)$, and the seventh most frequently isolated bloodstream pathogen (3\%) (Centers for Disease Control and Prevention, 2004). P. aeruginosa was also fifth overall in bacterial species responsible for nosocomial infections (9\%) (Centers for Disease Control and Prevention, 2004). In the past decade, P. aeruginos a has only increased in prevalence and 
antibiotic resistance, making this organism a definite threat for susceptible patients (Aloush et al., 2006; Edwards et al., 2007).

As an opportunistic pathogen, $P$. aeruginosa has the ability to colonize a number of diverse infection sites when the host immune system is compromised (Figure 1.10). This can occur in patients with a serious underlying condition such as cancer (Krcmery et al., 2006), AIDS (Meynard et al., 1999), or major burn wounds (Regules et al., 2007), or those with immune suppression from recent surgery or organ transplant (Botha et al., 2006; Defez et al., 2004; Ullah et al.). Neonates are also very susceptible to $P$. aeruginosa infection due to their underdeveloped immune system (Foca, 2002).

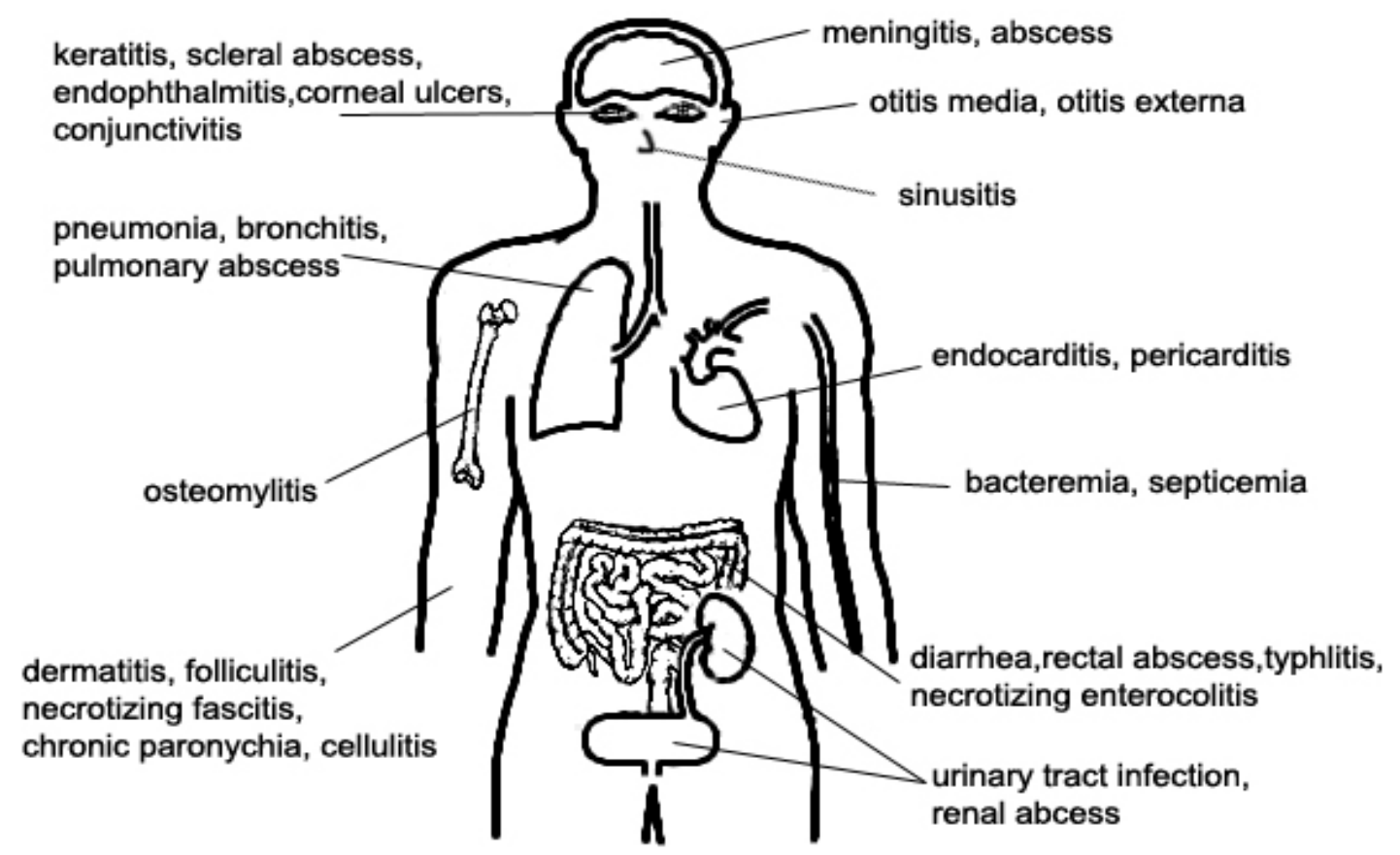

Figure 1.10 Various sites of $P$. aeruginosa infection 
P. aeruginosa can enter the body through any orifice; and minor infections, if left unchecked, can progress into severe, life-threatening conditions. In the ophthalmological system for instance, $P$. aeruginosa can colonize and infect the cornea, aqueous and vitreous humors, or surrounding structures after cataract or corrective surgery (Kehdi et al., 2005; Maccheron et al., 2004; Miller, 2000). Recently P. aeruginosa has been associated with an increasing number of cases of contact lens-associated kerititis (Robertson et al., 2007). Infections can progress quickly from minor conjunctivitis or kerititis to scleral lesions and corneal ulcers due to cell lysis by $P$. aeruginosa extracellular enzymes (Eifrig et al., 2003; Reedy \& Wood, 2000).

P. aeruginosa also colonizes the auditory canal, and infection can progress from minor otitis externa to inner ear complications (otitis media) (Clark et al., 1997; Roland, 2002). Without proper treatment secondary infections of the nearby bones (mastoiditis) or neurological structures can occur (Butbul-Aviel et al., 2003; Penido et al., 2005).

Disruption of the gastrointestinal system often occurs in pediatric patients or those with neutropenia or related blood disorders (Tsai et al., 1996). Colonization of the digestive tract can range from diarrhea to severe rectal lesions and necrotizing enterocolitis in these patients (Rowe et al., 1994).

Urinary tract infections (UTIs) are common in a hospital setting due to frequent catheterization and the presence of drug resistant bacteria (Nicolle, 2002). If left unchecked, these infections can lead to severe kidney disorders and renal failure. Proximal bone infections such as osteomylitis of the pelvis and lumbosacral vertebrae can occur as a secondary complication to UTIs (Sapico \& Montgomerie, 1990). 
P. aeruginosa infection can also occur following an abrasion or break in the skin, due to injury, surgery, or catheterization. Dermatological conditions range from minor events such as hot-tub dermatitis or folliculitis (Yu et al., 2007) to life threatening cases of necrotizing fascitis or cellulitis (Bodey et al., 1983; Tsekouras et al., 1998; Wortman, 1993). Infections with deep tissue damage especially in burn victims can spread to the bloodstream causing bacteremia and septicemia (Bodey et al., 1983). Bloodborne $P$. aeruginosa can travel to the heart in these cases causing endo- and pericarditis (Regules et al., 2007).

Complications in the pulmonary system can begin as sinusitis (Danielides et al., 2002; Koltai et al., 1985; Rombaux et al., 1997) or an upper respiratory tract infection and lead to chronic bronchitis, pneumonia, or pulmonary lesions (Boyer et al., 2005; Garau \& Gomez, 2003; Hausermann \& Gartmann, 1973; Zwillich \& Ellis, 1974). Infections of the sinuses can also lead to meningitis and cerebral lesions due to the close proximity to the brain (Glickstein et al., 2006; Weisfelt et al., 2007; Widerman, 1953)

\subsubsection{Pseudomonas aeruginosa and cystic fibrosis}

The most significant complications of the pulmonary system occur among patients with cystic fibrosis (CF), diffuse panbronchiolitis (DPB), and chronic obstructive pulmonary disease (COPD). These patients with reduced lung function are especially prone to chronic $P$. aeruginosa infection.

Cystic fibrosis is a terminal inherited disease among Caucasians, affecting 1 in 2500 live births in the United States (Registry, 2005). The gene responsible for CF, encodes the cystic fibrosis transmembrane conductance regulator (CFTR), a protein of 
approximately 1480 amino acids (Sheppard \& Welsh, 1999). Mutations in the CFTR result in decreased function of cyclic-AMP-activated chloride channels in secretory epithelial cells (Marcet \& Boeynaems, 2006). Decreased fluid production in the lung causes the dehydration of epithelial surfaces and oversecretion of mucus (Frizzell, 1999). Reduced aeration and lung clearance due to thick mucosa results in increased susceptibility to bronchopulmonary infection with $P$. aeruginosa among other organisms.

A switch to a mucoid phenotype presents even more problems for the CF patient (Henry et al., 1993; Pritt et al., 2007). Nonmucoid P. aeruginosa strains in the early stages of infection do little harm to CF lungs, and can usually be eradicated by early aggressive antibiotic therapy (Hoiby, 1993). However, the subsequent appearance of mucoid strains correlates with the formation of a bacterial biofilm, the development of anti- $P$. aeruginosa antibodies, inflammation, and a poor prognosis for the patient (Henry et al., 1993; Koch, 1993; Pederson, 1992; Stapper et al., 2004). Antibiotic treatment is switched to a rotation of various antibacterial agents but ultimately fails due to decreased lung function via bacterial consumption.

\subsubsection{Current treatment protocols}

Current treatment protocols can vary greatly since a patient may be infected with one or more drug resistant strains of $P$. aeruginosa. Therapeutic strategies to treat infection include administration of a single antibiotic or a combination of two or three anti-pseudomonal agents (Banerjee \& Stableforth, 2000; Jain \& Singh, 2007). Monotherapy has generally included treatment with $\beta$-lactam antibiotics such as penicillins, cephalosporins, or newer broad-spectrum $\beta$-lactams such as imipenem and 
meropenem (Banerjee \& Stableforth, 2000; Flanders et al., 2006). These antibiotics act by interfering with the organization of peptidoglycan into bacterial cell walls (Bycroft \& Shute, 1985) (Figure 1.11). Unfortunately, P. aeruginosa has evolved an efficient way of inactivating these drugs (discussed in the following section) which led to the development of a number of strategic combination therapies.

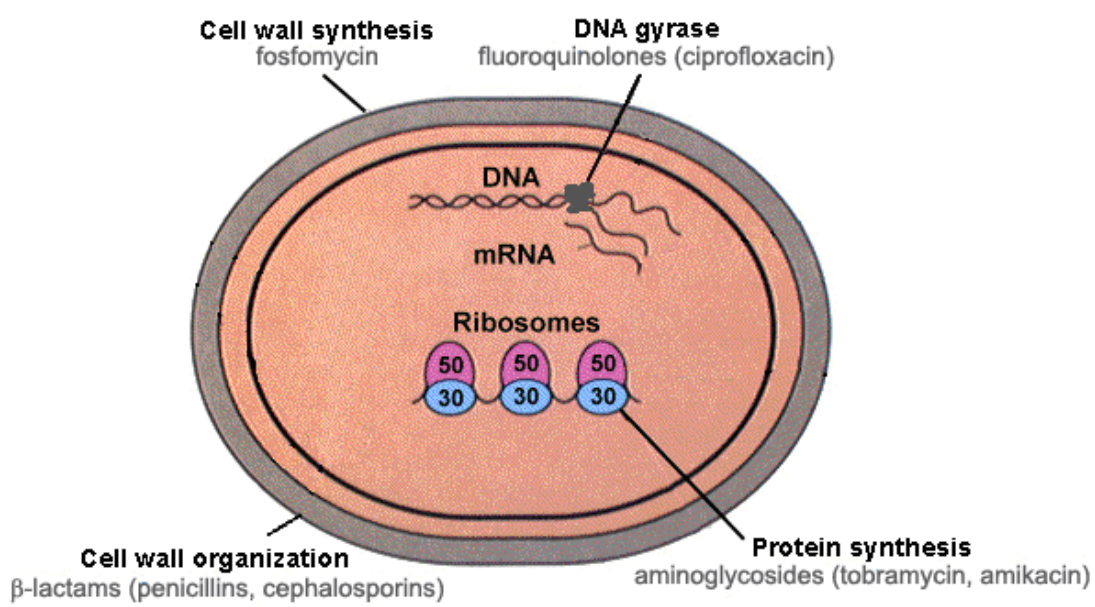

Figure 1.11 Common anti-pseudomonal antibiotics and mechanisms of action. Combinations of these drugs are often necessary for efficacy against resistant strains of $P$. aeruginosa. (Image Source: Kalai Mathee)

Anti-pseudomonal cocktails include combinations of a $\beta$-lactam such as ticarcillin, aztreonam or ceftazidime plus a $\beta$-lactamase inhibitor such as sulbactam, and an aminoglycoside antibiotic such as tobramycin or amikacin (Banerjee \& Stableforth, 2000; Flanders et al., 2006; Ostendorf et al., 2006). Aminoglycosides interfere with protein synthesis by binding to the ribosomal subunits of the bacterial cell (Hancock, 1981; Omri et al., 1995). Combinations of ceftazidime and a fluoroquinolone such as 
ciprofloxacin are also effective. The quinolones exhibit their bactericidal action by blocking DNA replication through gyrase inhibition (Hooper, 1999).

Alternative combinations include pairing ciprofloxacin with an animoglycoside or with the broad-spectrum antibiotic fosfomycin, which prevents proper cell wall formation by inhibiting production of $\mathrm{N}$-acetylmuramic acid (Flanders et al., 2006).

Although there is some success in eradicating $P$. aeruginosa with these treatments many CF patients need ongoing treatment and toxicity can develop with continual use (Banerjee \& Stableforth, 2000; Registry, 2005). In addition, there is an escalating trend of antibiotic resistance that will soon render these therapeutics ineffective.

\subsubsection{Antibiotic resistance in Pseudomonas aeruginosa}

According to 2004 data provided by the Centers for Disease Control, antibiotic resistant $P$. aeruginosa infections are increasing (Centers for Disease Control and Prevention, 2004). In $2004,21.1 \%$ of the nosocomial infections were resistant to imipenem, an increase of $15 \%$ over the previous five years (1998-2002). Similarly, $29.5 \%$ of hospital-acquired $P$. aeruginosa infections were resistant to quinolones and $31.9 \%$ were resistant to third generation cephalosporins; increases of $9 \%$ and $20 \%$ over the previous five years respectively (Centers for Disease Control and Prevention, 2004).

A hospital setting is ideal for the promotion of antibiotic resistance, either through overuse and subsequent mutation or via gene transfer (Gregory et al., 2008; Hancock \& Speert, 2000) (Figure 1.12). In addition to the more recently acquired resistance mechanisms $P$. aeruginosa also has several factors that are considered intrinsic. Current 
pathogenic strains of $P$. aeruginosa possess inventive mechanisms which contribute to reduced antibiotics susceptibility including:
A. Biofilm formation
B. Restricted surface accessibility
C. Exclusion via efflux pumps
D. Enzymatic inactivation of antibiotics
E. Alteration of target proteins

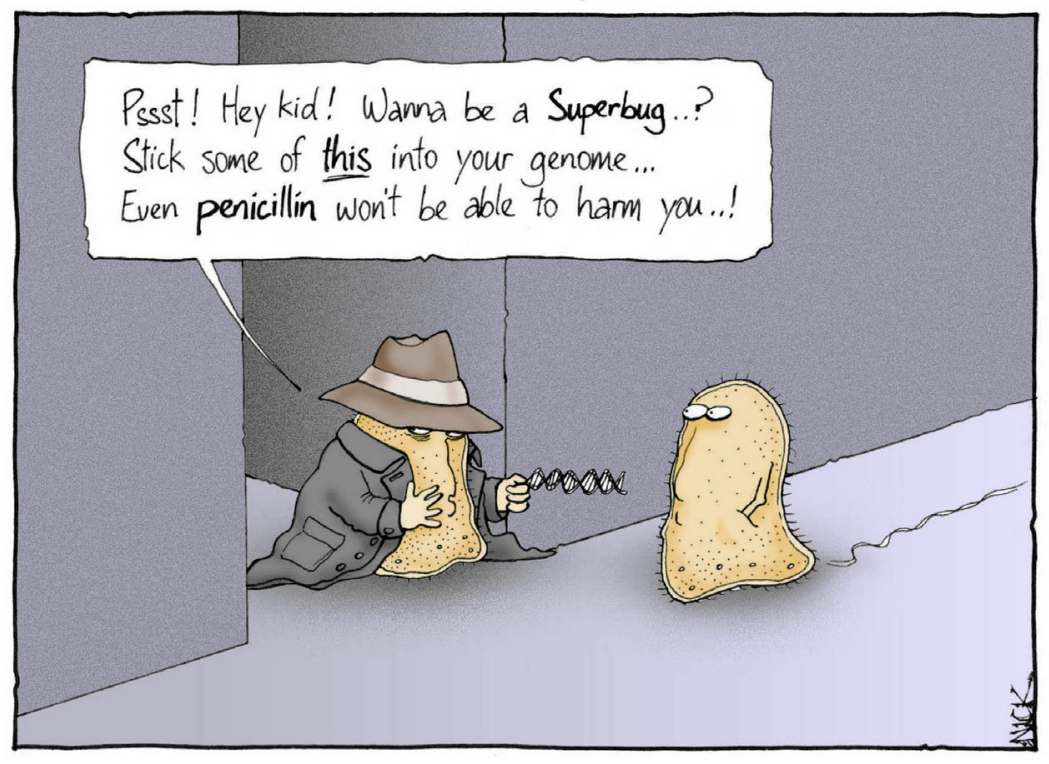

It was on a short-cut through the hospital kitchens that Albert was first approached by a member of the Antibiotic Resistance.

Figure 1.12 "Gene transfer". Cartoon by Nick D Kim. Used with permission. (http://www.nearingzero.net) 


\subsubsection{Biofilm formation}

"The rule of thumb is that 1,500 times more of an antimicrobial agent is needed to kill a biofilm than a planktonic bacteria." - William Costerton.

Although the planktonic mode of bacterial growth is most often studied, the majority of bacteria exist in nature as members of a surface-adherent, matrix-enclosed biofilm (Costerton et al., 1995) (Figure 1.13). P. aeruginosa is no exception to this rule, and often switches to a mucoid phenotype during infection (Mathee et al., 1999; Pritt et al., 2007; Stapper et al., 2004). Owing to the secretion of an exopolysaccharide, biofilm cells form a slime layer in which they are irreversibly bound to a substratum and to each other (Donlan \& Costerton, 2002). This protected formation results in altered growth rates and transcription patterns, and more importantly, an increased environmental resistance from that of their planktonic counterparts (Donlan \& Costerton, 2002). Antimicrobial agents are prevented from reaching the innermost cells of a biofilm and are therefore unable to fully eradicate the infection (Hoyle \& Costerton, 1991).

In addition, the alginate layer of mucoid $P$. aeruginosa prevents optimal host immune function by masking antibody opsonization and inhibiting clearance (Jensen, 1993; Jensen, 1990; Pier et al., 1995). The latter is accomplished by promoting irreversible adherence of the bacteria to lung epithelial cells. 


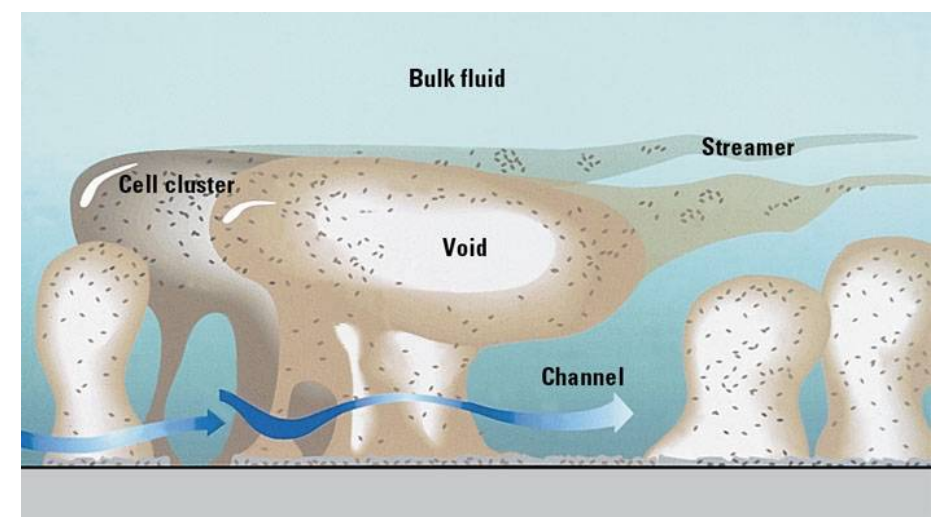

Figure 1.13 Biofilm formation. Polysaccharides secreted by $P$. aeruginosa help to adhere cells to a surface and to each other resulting in mushroom like structures, clusters, void spaces, water channels, and streamers. Peg Dirckx, Center for Biofilm Engineering. Used with permission. (http://www.erc.montana.edu)

\subsubsection{Restricted surface accessibility}

If an antibiotic does manage to penetrate the structural protection of the biofilm layer, $P$. aeruginosa has a second line of defense - a rather impermeable outer membrane. For an antibiotic to be effective, it must be able to reach the periplasm or the intercellular space of the bacterium. Outer membrane permeability is associated with a number of channel-like proteins, or porins, which act as gate-keepers for molecules wishing to gain entry into the cell. The outer membrane of P. aeruginosa is estimated to be up to 100 times less permeable than that of E. coli (Yoshimura \& Nikaido, 1982). This is due, in part, to the difference in size and structure of the porins. The outer membrane proteins (Oprs) found in $P$. aeruginosa had much lower molecular weights than their $E$. coli counterparts (Mizuno \& Kageyama, 1978; Yoshihara \& Nakae, 1989) and existed as 
monomers (Yoshimura et al., 1983), whereas the E. coli porins had a larger, trimeric quaternary structure. Modulation of permeability, and thus antibiotic susceptibility in $P$. aeruginosa, has been shown to occur with changes in expression or mutations of the Opr genes (Yoneyama et al., 1991; Yoneyama et al., 1995; Young et al., 1992).

\subsubsection{Exclusion via efflux pumps}

Once an antibiotic gains entry into the cell, $P$. aeruginosa has a number of threecomponent efflux mechanisms in place to remove it before it causes damage. The tripartite efflux system combines an outer membrane protein described in the previous section (e.g. OprM), an energy-dependant pump located in the cytoplasmic membrane (MexB), and a protein to connect the two (MexA)(Nehme et al., 2004). This system creates a channel which extends from the inner membrane all the way to the outside of the cell and uses proton motive force to extrude unwanted chemicals (Figure 1.14). $P$. aeruginosa expresses a number of these multidrug efflux (Mex) systems, including MexAB-OprM, MexXY-OprM, MexCD-OprJ, MexEF-OprN and MexJK-OprM (Chuanchuen et al., 2002; Hocquet et al., 2003; Llanes et al., 2004; Maseda et al., 2000; Masuda et al., 2000; Poole, 2001). These systems vary slightly in their preferred substrates, however their combined effect removes most known classes of antibiotics including $\beta$-lactams (and $\beta$-lactamase inhibitors), macrolides, aminoglycosides, fluoroquinolones, tetracyclines, and chloramphenicol (Poole \& Srikumar, 2001). Hyperexpression of these efflux genes by mutational events or acquired genetic material has been shown to result in substantially increased antibiotic resistance (Beinlich et al., 2001; Llanes et al., 2004). 


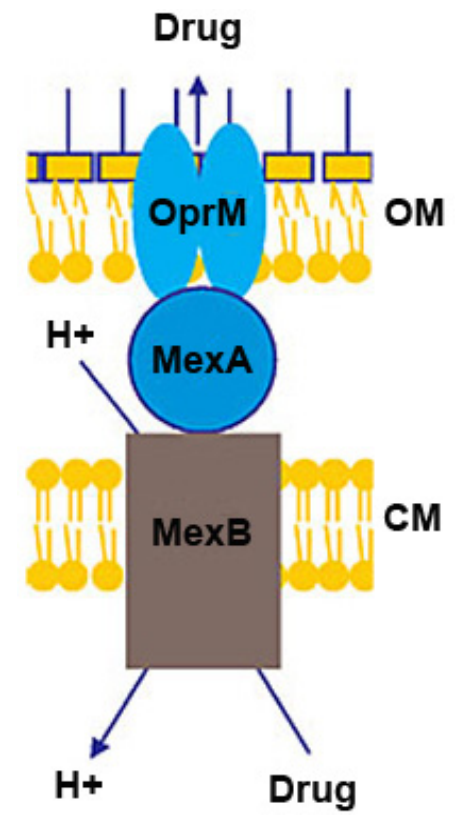

Figure 1.14 MexAB-OprM efflux pump of $P$. aeruginosa. This system extrudes drugs using proton motive force (modified from (Schweizer, 2003)). OM = outer membrane, $\mathrm{CM}=$ cytoplasmic membrane.

\subsubsection{Enzymatic inactivation of antibiotics}

$P$. aeruginosa is able to inactivate many $\beta$-lactam antibiotics by enzymatic cleaving of the $\beta$-lactam ring (Figure 1.15) (Abraham \& Chain, 1940). Older antibiotics in the $\beta$-lactam class such as ampicillin and penicillin have almost no effect on current strains of $P$. aeruginosa. Newer $\beta$-lactams are still used to treat infections with this organism, however, resistance is on the rise and soon these too will become obsolete.

$\beta$-lactamases are separated into four distinct classes based on structure, function, and substrate specificity (Abraham \& Chain, 1940). P. aeruginosa can carry one or more $\beta$-lactamases from any of the Ambler classes A-D (Ambler, 1980). 


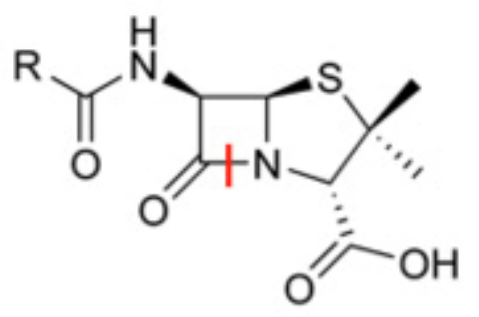

Figure 1.15 Core structure of Penicillin, typical of $\beta$-lactam antibiotics. Red line indicates site of cleavage by $\beta$-lactamases. (http://en.wikipedia.org/wiki/Image:Penicillincore.png)

Ambler class A $\beta$-lactamases, or penicillinases, were the first identified and most variable class of these enzymes (Matagne et al., 1990). They act via a serine hydrolasedriven mechanism, and generally have activity against the older penicillin derivatives. The most notable enzymes in this class are the chromsomally mediated TEM (isolated

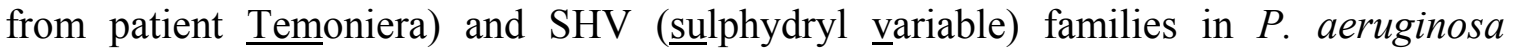
(Mugnier et al., 1996; Naas et al., 1999; Poirel et al., 1999) and the plasmid-encoded CARB, which confers carbenicillin resistance (Boissinot \& Levesque, 1990; Lachapelle et al., 1991). Class A $\beta$-lactamases generally lack activity against cephalosporins and carbepenems, however, a number of extended spectrum $\beta$-lactamases (ESBLs) are emerging throughout Europe and Asia (Weldhagen et al., 2003). These ESBLs have increased activity against several broad-spectrum antibiotics, severely limiting treatment options (Weldhagen et al., 2003).

Ambler class $\mathrm{B}$ enzymes are metallo- $\beta$-lactamases which require zinc for activation (Ambler, 1980; Bush et al., 1995). The first of these (IMP-1) was isolated from a Japanese strain of $P$. aeruginosa (Watanabe et al., 1991). IMP appears to be widely 
distributed throughout Gram negative bacteria and confers resistance not only to imipanem, but to advanced cephalosporins, penicillins, and several $\beta$-lactamase inhibitors as well (Watanabe et al., 1991). In addition, IMP genes are encoded on a class 1 integron on a plasmid conferring resistance to additional classes of antibiotics. The ease of intraand interspecies transfer of plasmids of this type has resulted in multi-drug resistant (MDR) strains of P. aeruginosa and other pathogenic organisms (Walsh et al., 2005). A second, class B enzyme with a strong resistance to carbapenem (VIM) is becoming increasingly present as well (Lauretti et al., 1999; Tsakris et al., 2000).

Class C $\beta$-lactamases, or cephalosporinases, like Class A enzymes use a serinehydrolase mechanism to cleave the $\beta$-lactam ring of invading drugs (Sanders, 1987). These chromosomally encoded enzymes are highly conserved (Galleni et al., 1988), and the most recognized enzyme, ampC from $P$. aeruginosa, has been cloned and sequenced (Lodge et al., 1990). Most class C $\beta$-lactamases including ampC are inducible in $P$. aeruginosa. They are produced at a basal level until the cell is challenged with a $\beta$-lactam antibiotic, at which point $P$. aeruginosa upregulates lactamase production, reduces its outer membrane permeability, and creates a resistant nonhydrolytic barrier (Sanders \& Sanders, 1985). This results in theraputic failure for not only the $\beta$-lactams but for other classes of drugs as well.

The Class $\mathrm{D}$ group of $\beta$-lactamases are structurally similar to Class A enzymes, but are functionally described as oxacillinases for their ablilty to degrade isoxazolyl $\beta$ lactams such as methicillin and oxacillin (Naas \& Nordmann, 1999). Oxacillinases (OXA family) are ubiquitous thoughout Gram negative bacteria and are generally plasmid- or 
transposon-encoded. However, a chomosomally encoded oxacillinase, PoxB, has also been characterized for P. aeruginosa (Girlich et al., 2004; Kong et al., 2005). As with Class A, treatment-compromising Class D ESBLs are becoming more prevalent in $P$. aeruginosa infections (Poirel, 2002).

\subsubsection{Alteration of target proteins}

P. aeruginosa has yet another method for dealing with $\beta$-lactam antibiotics - the expression of modified penicillin binding proteins (PBPs). Normal PBPs are membranebound or cytoplasmic proteins, that play a role in peptidoglycan and cell wall synthesis (Georgopapadakou \& Liu, 1980; Noguchi et al., 1979). Penicillin and related $\beta$-lactams need to bind these PBP targets to exhibit their antibiotic effect. Mutations in PBPs decrease binding affinity to the target and subsequently antibiotic susceptibility (Curtis et al., 1981; Noguchi et al., 1980). P. aeruginosa synthesizes at least nine divergent PBPs (Georgopapadakou \& Liu, 1980; Stover et al., 2000) which have a reduced binding affinity to penicillin when compared to those in E. coli (Curtis et al., 1979). In addition, a number of mutations have been found in clinically-isolated strains with increased resistance to imipenem (Bellido et al., 1990), titarcillin and piperacillin (Godfrey et al., 1981), and cefsulodin (Gotoh et al., 1990). Still further accumulation of mutations in a hospital setting will add new complications to antibiotic treatment.

\subsubsection{Pseudomonas aeruginosa QS specifics}

With the difficulty in treating recalcitrant infections in the CF patient and the growing resistance to antibiotics, new therapeutic modalities are becoming increasingly 
necessary. Targeting the QS system of P. aeruginosa, one of the major complicating pathogens in the CF lung, is a novel plan of attack. This system is a key regulator of pathogenicity in $P$. aeruginosa and other medically relevant bacteria, thus inhibition of QS may cause attenuation of virulence and protect against infection (Hentzer \& Givskov, 2003; Juhas et al., 2005; Smith \& Iglewski, 2003a).

The main QS system of $P$. aeruginosa is based on the luxI-luxR paradigm described previously. This intricate communication system is mirrored in many gramnegative pathogenic bacteria, where it coordinates regulation of virulence, including motility, biofilm formation, and toxin production (for a review see (De Kievit \& Iglewski, 2000; Fuqua \& Greenberg, 1998; Schauder \& Bassler, 2001)). P. aeruginosa elaborates two main sets of QS systems: lasI-lasR and rhlI-rhlR (Schuster \& Greenberg, 2006). The LuxI homologues, LasI and RhlI are synthetases that manufacture the autoinducer signaling molecules N-(3-oxododecanoyl)-L-homoserine lactone (OdDHL) and N-butanoyl-L-homoserine lactone (BHL), respectively (Pearson et al., 1994; Pearson et al., 1995). These signaling molecules diffuse out into the environment and, upon reaching a putative threshold concentration, activate receptors las $R$ and rhlR (Pesci et al., 1997). These receptors, in turn, coordinate regulation of pathogenicity through transcriptional activation of a number of virulence factors (Brint \& Ohman, 1995; Latifi et al., 1995; Winson et al., 1995).

A third signal, PQS (Pseudomonas Quinolone Signal), also plays an integral role in the QS system and is implicated in the production of $N$-(3-oxohexanoyl)-L-homoserine lactone (OHHL) (McKnight et al., 2000; Pesci et al., 1999). This secondary metabolite of P. aeruginosa is incorporated into the QS hierarchy in times of cell stress (McKnight et 
al., 2000), and interference with this signal has been shown inhibit virulence factor expression (Calfee et al., 2001) It has also been suggested that a fourth system exists regulated by cyclic dipeptides (DKPs) (Holden, 1999). However, due to time constraints, this study focuses on the well-defined las and $r h l$ systems.

Although the redundant and auto-regulatory nature of the QS system is quite complex (Van Delden \& Iglewski, 1998), the basic view of the P. aeruginosa QS hierarchy suggests that las controls $r h l$ with virulence proteins at the bottom of the ladder (Schuster \& Greenberg, 2006) (Figure 1.16). The virulence factors LasA (staphylolytic protease) and LasB (elastase) are thought to be under control of the lasI/R system (Gambello, 1991; Storey et al., 1998), however rhlI/R also controls activity to a lesser extent (Brint \& Ohman, 1995; Pearson et al., 1997). Pyoverdin is believed to be under rhll/R control (Brint \& Ohman, 1995; Whiteley, 1999), whereas biofilm production is only partially under QS control (Davies et al., 1998). The las-rhl system also falls under the umbrella of a number of "global" regulators such as Vfr (a homologue of E. coli cAMP receptor protein, CRP) (Albus et al., 1997) or GacA (a sRNA binding protein) (Reimmann et al., 1997). Control from these genes also affects downstream virulence.

Thus, to inhibit pathogenicity, a therapeutic compound could exhibit an effect directly on the las/rhl system, one of the global regulators, or the PQS or DKP pathways. The only known anti-QS compounds, the halogenated furanones, inhibit $P$. aeruginosa both in vitro and in a murine model (Hentzer et al., 2002; Hentzer et al., 2003; Wu et al., 2004). These compounds function by displacing the signaling molecule from its receptor, thus accelerating receptor turnover (Manefield et al., 1999; Manefield et al., 2002). They also have an influence on siderophore biosynthesis (Ren et al., 2005). 


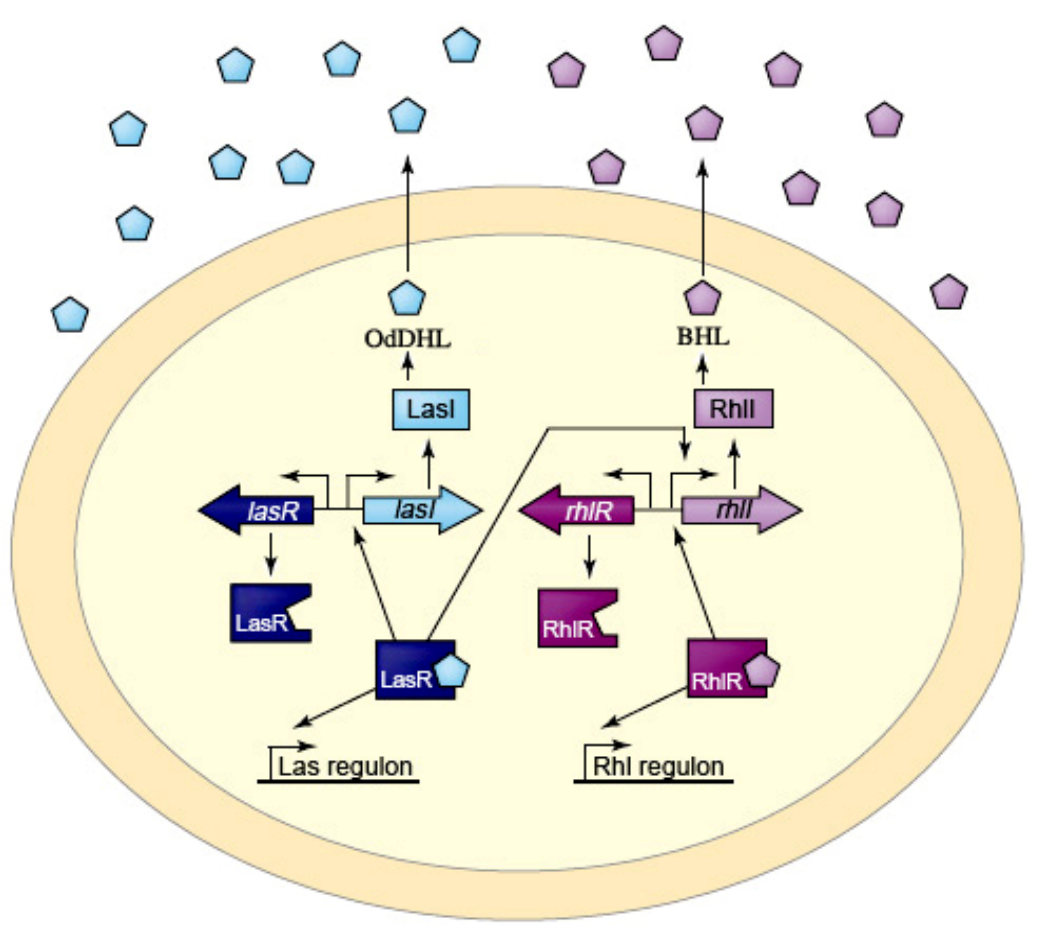

Figure 1.16 The main QS system in P. aeruginosa (modified from (Schuster \& Greenberg, 2006)). LasI and RhlI synthetases manufacture the autoinducer signaling molecules OdDHL and BHL respectively. These signaling molecules diffuse out into the environment and, upon reaching a threshold concentration, activate receptors las $R$ and $r h l R$. These receptors each control a regulon of genes including virulence factors.

Recently, a few plants have shown anti-QS activity against $P$. aeruginosa (Rasmussen et al., 2005), however the responsible compounds and mechanism of action are yet unknown. The potential for finding anti-pseudomonal and anti-QS activity in medicinal plants is considerable, relatively unexplored, and will be the focus of this study. 


\subsection{Infection model: Caenorhabditis elegans}

In vivo activity is often quite different than in vitro effect, and thus an animal model is a necessary step closer to therapeutic relevance. Significant to this study, are the number of $P$. aeruginosa virulence factors relevant in the killing of both nematode and mammalian tissues (Rahme et al., 1995; Tan et al., 1999a).

The nematode Caenorhabditis elegans (Figure 1.17) was developed as an animal model in the mid 1960s by Sidney Brenner (Brenner, 1974). With its small chromosome number and only 556 cells at hatching, C. elegans was posed as an alternative to the larger, more complex Drosophila model. The $1 \mathrm{~mm}$ long roundworm is a selfreproducing hermaphrodite with a life-cycle of approximately 3.5 days (Brenner, 1974). It lends itself easily to culture and propagation on Escherichia coli strain OP50, and hundreds of progeny can be generated rapidly in petri dishes.

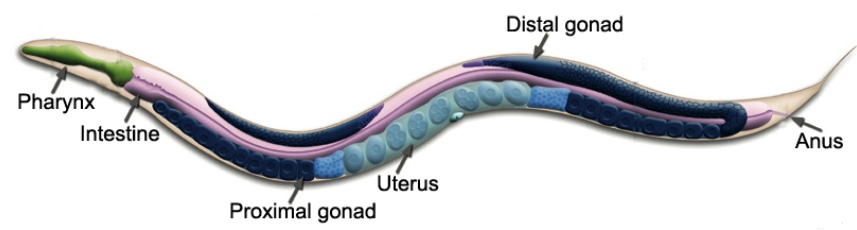

Figure 1.17 Caenorhabditis elegans. (Modified from http://www.wormatlas.org)

Although originally chosen for genetic and neurological study, C. elegans was later established as a practical model for studying bacterial virulence (Darby et al., 1999; Tan \& Ausubel, 2000). In addition to its preferred meal of E. coli, this voracious nematode will consume almost any bacteria that can fit down its pharynx, resulting in intestinal infection and worm death with a number of pathogenic species. 
Three major models of infection have been developed for exploring the effect of P. aeruginosa on C. elegans; "fast-killing", "slow-killing", and "paralytic" models. Fastkilling of C. elegans occurs within hours of incubation with P. aeruginosa strain PA14 on rich media (Mahajan-Miklos et al., 1999). The high-nutrient media allows maximum production of bacterial virulence factors such as phenazines and other toxins, which are absorbed through the hypoderm, or skin, of C. elegans. Slow-killing occurs over a period of days via ingestion of bacteria and subsequent infection, when the nematode is incubated with strain PA14 on minimal media (Tan et al., 1999a; Tan et al., 1999b). Paralytic killing is seen with the related P. aeruginosa strain PAO1, which causes death within hours through cyanide poisoning and neuromuscular paralysis (Gallagher \& Manoil, 2001).

Relevant to this study, is that $P$. aeruginosa-mediated killing of $C$. elegans in all of these cases is dependent on the QS system (Darby et al., 1999; Mahajan-Miklos et al., 1999; Tan et al., 1999b). Since virulence has been shown both in vitro and in a nematode model to be mediated by the bacterial QS system, the addition of plant compounds that attenuate $P$. aeruginosa $\mathrm{QS}$, should therefore decrease virulence factor production and subsequent death of C. elegans.

An additional advantage in using a live animal model for screening of potential anti-infective compounds is that both efficacy and host toxicity of a plant extract can be tested concurrently. This approach can be expanded to the screening of natural product libraries to search for compounds that cause a marked decrease in $P$. aeruginosa killing of C. elegans, without affecting worm fitness on E. coli. 


\subsection{Main hypotheses and aims of this project}

Hypothesis: Anti-QS compounds may contribute to the anti-infective properties of some traditionally used plants, and that these compounds can affect virulence and biofilm formation in human pathogenic organisms such as $P$. aeruginosa. Furthermore, extracts from plants with anti-QS properties should prevent infection and death in a live animal model system.

Specifically this research will explore the following aims:

I. Investigate the presence of anti-QS activity in south Florida medicinal plants.

II. Determine if these plants can affect the QS system and virulence factors of P. aeruginosa.

III. Test the ability of anti-QS plants to prevent infection in the model organism C. elegans.

IV. Purify and characterize the active compounds in these plant species.

The following research introduces not only a new mode of action and possible validation for traditional plant use, but also a new therapeutic direction for $\mathrm{CF}$ and other conditions complicated by bacterial infection. 


\section{Chapter 2}

"Anti-quorum sensing activity of medicinal plants in southern Florida"

As published in the Journal for Ethnopharmacology

January 2006

Volume 105, Pages 427-435

Copyright (C) Elsevier

Allison L. Adonizio, Kelsey Downum, Bradley C. Bennett, Kalai Mathee 


\subsection{Abstract}

Bacterial intercellular communication, or quorum sensing (QS), controls the pathogenesis of many medically important organisms. Anti-QS compounds are known to exist in marine algae and have the ability to attenuate bacterial pathogenicity. We hypothesized that terrestrial plants traditionally used as medicines may also produce antiQS compounds. To test this hypothesis, 50 medicinal plants from southern Florida were screened for anti-QS activity using two biomonitor strains, Chromobacterium violaceum and Agrobacterium tumefaciens. Of these plants, six showed QS inhibition: Conocarpus erectus L. (Combretaceae), Chamaecyce hypericifolia (L.) Millsp. (Euphorbiaceae), Callistemon viminalis (Sol.ex Gaertn.) G. Don (Myrtaceae), Bucida burceras L. (Combretaceae), Tetrazygia bicolor (Mill.) Cogn. (Melastomataceae), and Quercus virginiana Mill. (Fagaceae). This study introduces not only a new mode of action and possible validation for traditional plant use, but also a potentially new therapeutic direction for the treatment of bacterial infections.

Keywords: Medicinal plants; Antimicrobial activity; Quorum sensing; Anti-quorum sensing; Southern Florida, Anti-infective potential

\footnotetext{
Abbreviations: QS, Quorum Sensing; AHL, Acyl-Homoserine Lactone; X-gal, 5-bromo4-chloro-3-indoyl- $\beta$-D-galactopyranoside; WT, Wild Type
} 


\subsection{Introduction}

Whether for traditional use-validation or drug discovery purposes, previous studies have focused on the antibacterial potential of medicinal plants (e.g.: (Cowan, 1999; Wallace, 2004)). Investigations were restricted to whether or not a plant could kill or inhibit the growth of bacteria. However, this is only one facet of a plant's antiinfective potential. The antipathogenic properties of plants have received much less attention, but may be just as important in combating disease as their antibacterial counterparts. The interruption of quorum sensing (QS), or bacterial cell-to-cell communication, is one example of an antipathogenic effect. Since a large number of systems affecting pathogenicity are controlled by QS, interrupting this communication system can render pathogenic bacteria non-virulent (Zhang \& Dong, 2004).

Quorum sensing (QS) is a population-dependent phenomenon first characterized in the 1970s in luminescent marine species of Vibrio (Hastings \& Greenberg, 1999; Nealson et al., 1970). QS systems are ubiquitous in bacteria, and have since been found to regulate diverse cellular functions including luminescence, biofilm formation, antibiotic production, virulence factor expression, pigment production, plant-microbe interactions, and motility (Fuqua \& Greenberg, 2002; Whitehead et al., 2001a). In QS, small signaling molecules called autoinducers mediate the ability to sense the size of a bacterial population (Eberhard et al., 1981). Autoinducers are constantly produced and received at a basal level by bacterial cells. With high population density, there is a surplus of such molecules in the environment (Hastings \& Greenberg, 1999). These molecules then interact with a transcriptional regulator to activate the expression of genes involved in light production in luminescent Vibrio species (Fuqua et al., 1994). In other 
bacteria, these chemical signals are important for the establishment of infection and can serve as a switch to a pathogenic state (De Kievit \& Iglewski, 2000; Sircili et al., 2004; Wu et al., 2001).

Several signaling molecules have been identified (Dong \& Zhang, 2005); the bestcharacterized being the acyl-homoserine lactones (AHLs) in Gram-negative bacteria (Eberhard et al., 1981). The AHLs are highly conserved, having the same homoserine lactone moiety but different acyl side chains and substitution (carbonyl or hydroxyl) at the C3 carbon (Fuqua \& Greenberg, 2002). AHL-mediated quorum sensing systems have been characterized in bacteria associated with human disease, e.g.: Pseudomonas aeruginosa (Smith \& Iglewski, 2003b), Yersinia pseudotuberculosis (Atkinson et al., 1999), Clostridium difficile (Carter et al., 2005), Burkholderia cepacia (Lutter et al., 2001) and Escherichia coli (Surette \& Bassler, 1998); as well as plant associated bacteria e.g.: Rhizobium leguminosarum (Rodelas et al., 1999), Ralstonia solanacearum, and Erwinia carotovora (Von Bodman et al., 2003). The discovery of the QS system and its critical role in bacterial virulence and survival has revealed a new target - a novel way to attack and attenuate bacterial pathogenicity.

There are a number of ways to interrupt the QS system. Thus anti-quorum sensing (anti-QS) compounds can be of great interest in the treatment of bacterial infections (Fast, 2003; Rice et al., 2005). A number of quorum-quenching enzymes that hydrolyze AHLs have been identified in bacteria (Dong \& Zhang, 2005). To date, the only known anti-QS compounds of non-bacterial origin are halogenated furanones from the red alga Delisea pulchra (Manefield et al., 1999). Anti-QS activity has also been shown in a number of southern Florida seaweeds (Cumberbatch, 2002) and a few terrestrial plants (Gao et al., 
2003; Teplitski et al., 2000). However, so far, only a handful of higher plants have been studied, and nothing has been published with regard to anti-QS activity in medicinal plants.

The plant kingdom has long been a source of medicines and is estimated to provide over half of today's pharmaceuticals (Balandrin et al., 1993). The emergence of antibiotic resistance begs the need for novel therapeutics. It has been suggested that targeting the QS system, instead of killing bacteria, may provide a solution to antibiotic resistance (Hentzer \& Givskov, 2003b). With the promise of anti-QS compounds, one should be compelled to search for these agents by the most efficient method possible. There have been many ethnobotanically-directed searches for agents to treat infection, demonstrating not only the need for these drugs, but also the large number of plants utilized for bacterial conditions (e.g.: (Camporese et al., 2003; Cowan, 1999; Gnanamani et al., 2003; Hernandez et al., 2003)). Although this antibacterial effect is important, it is not the only source of a plant's medicinal properties. Shifting the focus from the strictly antibacterial to anti-QS properties of plants may reveal new anti-infective compounds and provide use validation for traditional medicinals.

Using two different biomonitor strains selected for their QS genetics, we were able to screen southern Florida plants for anti-QS activity. We have found anti-QS activity in six out of 50 plants, and in most cases this activity is retained upon extraction into common solvents. 


\subsection{Materials and methods}

\subsubsection{Collection of plant material and extract preparation}

Fifty plant species (Table 2.1) were collected in the Florida International University Environmental Preserve and Miami-Dade County, FL from February-May 2004. Plant selection was based on availability and ethnobotanical use categories relating to infection (Burkhill, 1985; Duke, 1972; Duke, 1985; Duke, 2000; Liogier, 1990; Morton, 1981). These categories include plants used to treat wounds, burns, and dermatitis; conditions that favor microbial colonization. Plants were collected and processed in triplicate. All plants were identified by the authors and vouchers were deposited in the herbarium at Fairchild Tropical Botanic Garden (FTG), Miami, FL.

As a preliminary screen, unwashed leaves from 10 species were compared with leaves washed in ethanol to determine the effect of surface microbes and epiphylls. Plants were then processed and tested in four stages using two biomonitor strains (described in the following section). Testing of fresh material composed the primary screen, as it was faster and ethnomedicinally analogous to poultices. Plants that showed anti-QS activity as fresh material were tested in dried form. Those that still retained activity after drying were tested as water and ethanol extractions. Screening was performed in this manner, rather than testing extracts only, since certain chemicals may have been lost or altered during processing.

For ethanolic extracts, plants were separated into component parts and dried in a plant drier for approximately for $24 \mathrm{~h}$. Dried plant matter was ground and added to $95 \%$ ethanol (100 g dry wt/ L), and allowed to stand for 24 hours before vacuum filtration with filter paper (Whatman, 1001-270, Florham Park, NJ) to remove particulate matter. An 
aliquot was removed to test for anti-QS activity at the $100 \mathrm{~g} / \mathrm{L}$ concentration. The remainder was evaporated to dryness using a rotoevaporator (Buechi R-114, Uster, Switzerland) and stored at $-20^{\circ} \mathrm{C}$.

In addition, water extracts were prepared for the six species that proved active as fresh material. Plants were processed as above, dried material was added to sterile water at $100 \mathrm{~g}$ dry $\mathrm{wt} / \mathrm{L}$, and boiled for $5 \mathrm{~min}$. An aliquot was removed for testing and the remainder of the decoction was frozen at $-80{ }^{\circ} \mathrm{C}$ for 24 hours and then freeze-dried using a lyophilizer. The lyophilized extracts remained at $-20{ }^{\circ} \mathrm{C}$ to be reconstituted in sterile water as needed. Filtration of the water extracts using a $0.2 \mu \mathrm{m}$ (pore size) filter into autoclaved vials ensured sterility of the samples. Extracts were tested for microbial contamination at every step of processing, by streaking to LB agar plates, to minimize the potential for introduction of exogenous anti-QS compounds.

Table 2.1 Southern Florida medicinal plants tested for anti-QS activity.

\begin{tabular}{|l|l|l|l|l|l|}
\hline $\begin{array}{c}\text { Collection } \\
\#\end{array}$ & \multicolumn{1}{|c|}{ Species $^{\text {a }}$} & \multicolumn{1}{|c|}{ Family } & \multicolumn{1}{c|}{$\begin{array}{c}\text { Common } \\
\text { name }\end{array}$} & \multicolumn{1}{|c|}{ Part tested } & \multicolumn{1}{|c|}{ Medicinal use } \\
\hline ALA49 & $\begin{array}{l}\text { Asclepias } \\
\text { curassavica } \text { L. }\end{array}$ & Apocynaceae & $\begin{array}{l}\text { scarlet } \\
\text { milkweed }\end{array}$ & flowers, leaves & $\begin{array}{l}\text { emetic, } \\
\text { vermifuge, skin } \\
\text { disease, } \\
\text { leucorrhea }\end{array}$ \\
\hline ALA3 & $\begin{array}{l}\text { Bauhinia } \\
\text { variegata } \text { L. }\end{array}$ & Fabaceae & orchid tree & flowers, leaves & $\begin{array}{l}\text { other spp. asthma, } \\
\text { venereal disease, } \\
\text { influenza }\end{array}$ \\
\hline ALA4 & $\begin{array}{l}\text { Bidens alba } \text { var. } \\
\text { radiata (Sch. Bip.) } \\
\text { R.E. Ballard }\end{array}$ & Asteraceae & $\begin{array}{l}\text { beggarticks, } \\
\text { romerillo }\end{array}$ & aerial & $\begin{array}{l}\text { Bidens pilosa } \text { L. } \\
\text { for dysentery, } \\
\text { catarrh, } \\
\text { vermifuge, } \\
\text { wounds, sores }\end{array}$ \\
\hline ALA30 & $\begin{array}{l}\text { Bougainvillea } \\
\text { spectabilis } \text { Willd. }\end{array}$ & Nyctaginaceae & bougainvillea & flowers, leaves & $\begin{array}{l}\text { other spp. } \\
\text { wounds, cough }\end{array}$ \\
\hline
\end{tabular}




\begin{tabular}{|c|c|c|c|c|c|}
\hline ALA31 & Bucida buceras L. & Combretaceae & black olive & leaves & gonorrhea \\
\hline ALA21 & $\begin{array}{l}\text { Bursera simaruba } \\
\text { (L.) Sarg. }\end{array}$ & Burseraceae & gumbo-limbo & bark & $\begin{array}{l}\text { fever, venereal } \\
\text { disease, diarrhea, } \\
\text { skin disease, sore } \\
\text { throat, colds, } \\
\text { asthma }\end{array}$ \\
\hline ALA20 & $\begin{array}{l}\text { Callistemon } \\
\text { viminalis (Sol. ex } \\
\text { Gaertn.) G. Don }\end{array}$ & Myrtaceae & bottlebrush & $\begin{array}{l}\text { flowers/bracts, } \\
\text { leaves }\end{array}$ & $\begin{array}{l}\text { Melaleuca spp. } \\
\text { antiseptic, acne, } \\
\text { bronchitis, cough, } \\
\text { diarrhea, skin } \\
\text { disorders }\end{array}$ \\
\hline ALA5 & $\begin{array}{l}\text { Casuarina } \\
\text { equisetifolia } \mathrm{L} .\end{array}$ & Casuarinaceae & $\begin{array}{l}\text { Australian } \\
\text { pine }\end{array}$ & leaves & $\begin{array}{l}\text { skin disorders, } \\
\text { diarrhea, } \\
\text { dysentery, sore } \\
\text { throat }\end{array}$ \\
\hline ALA6 & $\begin{array}{l}\text { Catharanthus } \\
\text { roseus (L.) G. } \\
\text { Don }\end{array}$ & Apocynaceae & $\begin{array}{l}\text { Madagascar } \\
\text { periwinkle }\end{array}$ & flower, leaves & $\begin{array}{l}\text { asthma, } \\
\text { flatulence, } \\
\text { opthalmia, } \\
\text { tuberculosis, } \\
\text { vermifuge, } \\
\text { mouthwash }\end{array}$ \\
\hline ALA10 & $\begin{array}{l}\text { Chamaesyce } \\
\text { hypericifolia (L.) } \\
\text { Millsp. }\end{array}$ & Euphorbiaceae & $\begin{array}{l}\text { graceful } \\
\text { sandmat }\end{array}$ & aerial & $\begin{array}{l}\text { abrasions, } \\
\text { diarrhea, } \\
\text { dysentery, } \\
\text { leucorrhea, } \\
\text { opthalmia, } \\
\text { respiratory } \\
\text { problems }\end{array}$ \\
\hline ALA12 & $\begin{array}{l}\text { Chrysobalanus } \\
\text { icaco L. }\end{array}$ & Chrysobalanaceae & cocoplum & leaves & $\begin{array}{l}\text { dysentery, } \\
\text { leucorrhea, } \\
\text { diarrhea }\end{array}$ \\
\hline ALA13 & $\begin{array}{l}\text { Coccoloba uvifera } \\
\text { (L.) L. }\end{array}$ & Polygonaceae & seagrape & leaves & $\begin{array}{l}\text { dysentery, } \\
\text { diarrhea, asthma, } \\
\text { wounds, skin } \\
\text { disease }\end{array}$ \\
\hline ALA14 & $\begin{array}{l}\text { Conocarpus } \\
\text { erectus L. }\end{array}$ & Combretaceae & buttonwood & leaves & $\begin{array}{l}\text { febrifuge, } \\
\text { respiratory } \\
\text { ailments, venereal } \\
\text { disease }\end{array}$ \\
\hline ALA37 & $\begin{array}{l}\text { Costus } \\
\text { pulverulentus } \\
\text { C. Presl }\end{array}$ & Costaceae & costus & $\begin{array}{l}\text { flowers/bracts, } \\
\text { leaves, rhizome }\end{array}$ & $\begin{array}{l}\text { cough, dysentery, } \\
\text { venereal disease }\end{array}$ \\
\hline ALA17 & $\begin{array}{l}\text { Crotalaria pumila } \\
\text { Ortega }\end{array}$ & Fabaceae & low rattlebox & aerial & $\begin{array}{l}\text { Crotalaria incana } \\
\text { L., Crotalaria } \\
\text { fulva Roxb. for } \\
\text { yellow fever, rash }\end{array}$ \\
\hline
\end{tabular}




\begin{tabular}{|c|c|c|c|c|c|}
\hline ALA51 & $\begin{array}{l}\text { Cymbopogon } \\
\text { citratus (DC.) } \\
\text { Stapf }\end{array}$ & Poaceae & lemongrass & leaves & $\begin{array}{l}\text { stomachic, fever, } \\
\text { colds, } \\
\text { tuberculosis }\end{array}$ \\
\hline ALA32 & $\begin{array}{l}\text { Datura metel L. } \\
\text { "Double Purple" } \\
\text { c.v. }\end{array}$ & Solanaceae & $\begin{array}{l}\text { angel's } \\
\text { trumpet }\end{array}$ & flowers, leaves & $\begin{array}{l}\text { febrifuge, } \\
\text { stomachache, } \\
\text { asthma }\end{array}$ \\
\hline ALA33 & $\begin{array}{l}\text { Emilia fosbergii } \\
\text { Nicolson }\end{array}$ & Asteraceae & $\begin{array}{l}\text { florida } \\
\text { tassleflower }\end{array}$ & whole plant & $\begin{array}{l}\text { Emilia coccinea } \\
\text { (Sims) G. Don for } \\
\text { opthalmia, } \\
\text { asthma, } \\
\text { stomachache }\end{array}$ \\
\hline ALA18 & $\begin{array}{l}\text { Erigeron } \\
\text { quercifolius Lam. }\end{array}$ & Asteraceae & $\begin{array}{l}\text { oakleaf } \\
\text { fleabane }\end{array}$ & aerial & $\begin{array}{l}\text { Erigeron } \\
\text { karvinskianus } \\
\text { D.C. as cold } \\
\text { remedy }\end{array}$ \\
\hline ALA15 & Ficus aurea Nutt. & Moraceae & strangler fig & leaves, latex & coughs \\
\hline ALA16 & $\begin{array}{l}\text { Hamelia patens } \\
\text { Jacq. }\end{array}$ & Rubiaceae & firebush & flowers, leaves & $\begin{array}{l}\text { dysentery, skin } \\
\text { disease, sores, } \\
\text { blisters, eczema, } \\
\text { stomachache }\end{array}$ \\
\hline ALA19 & $\begin{array}{l}\text { Heliotropium } \\
\text { polyphyllum } \\
\text { Lehm. }\end{array}$ & Boraginaceae & $\begin{array}{l}\text { pineland } \\
\text { heliotrope }\end{array}$ & aerial & $\begin{array}{l}\text { other spp. sore } \\
\text { throat, asthma, } \\
\text { cough, bronchitis, } \\
\text { dysentery, eye } \\
\text { wash }\end{array}$ \\
\hline ALA38 & $\begin{array}{l}\text { Hibiscus rosa- } \\
\text { sinensis L. }\end{array}$ & Malvaceae & $\begin{array}{l}\text { garden } \\
\text { rosemallow, } \\
\text { hibiscus }\end{array}$ & flowers & $\begin{array}{l}\text { cold remedy, } \\
\text { influenza, } \\
\text { asthma, } \\
\text { opthalmia, } \\
\text { wounds }\end{array}$ \\
\hline ALA34 & Ixora coccinea $\mathrm{L}$. & Rubiaceae & $\begin{array}{l}\text { scarlet } \\
\text { jungleflame }\end{array}$ & flowers, leaves & $\begin{array}{l}\text { bronchitis, } \\
\text { catarrh, diarrhea, } \\
\text { fever, sore }\end{array}$ \\
\hline ALA7 & $\begin{array}{l}\text { Lantana depressa } \\
\text { L. }\end{array}$ & Verbenaceae & $\begin{array}{l}\text { rockland } \\
\text { lantana }\end{array}$ & flowers, leaves & $\begin{array}{l}\text { stomachic, } \\
\text { wound, colds, } \\
\text { fever, asthma, } \\
\text { veneral disease, } \\
\text { bronhitis }\end{array}$ \\
\hline ALA40 & $\begin{array}{l}\text { Lepidium } \\
\text { virginicum } \mathrm{L} .\end{array}$ & Brassicaceae & pepperweed & aerial & $\begin{array}{l}\text { mouth sores, skin } \\
\text { disease, stomach } \\
\text { ailments, } \\
\text { vermifuge, } \\
\text { diarrhea, } \\
\text { dysentery }\end{array}$ \\
\hline
\end{tabular}




\begin{tabular}{|c|c|c|c|c|c|}
\hline ALA1 & $\begin{array}{l}\text { Oxalis corniculata } \\
\text { L. }\end{array}$ & Oxalidaceae & $\begin{array}{l}\text { yellow } \\
\text { woodsorrel }\end{array}$ & leaves & $\begin{array}{l}\text { skin conditions, } \\
\text { antiseptic, sore } \\
\text { throat, stomach } \\
\text { problems }\end{array}$ \\
\hline ALA23 & $\begin{array}{l}\text { Parthenocissus } \\
\text { quinquefolia (L.) } \\
\text { Planch. }\end{array}$ & Vitaceae & $\begin{array}{l}\text { Virginia } \\
\text { creeper }\end{array}$ & aerial & $\begin{array}{l}\text { antiseptic, fever, } \\
\text { diarrhea }\end{array}$ \\
\hline ALA42 & $\begin{array}{l}\text { Passiflora } \\
\text { coccinea Aubl. }\end{array}$ & Passifloraceae & $\begin{array}{l}\text { red passion } \\
\text { vine }\end{array}$ & flowers, leaves & $\begin{array}{l}\text { fever, } \\
\text { conjunctivitis }\end{array}$ \\
\hline ALA24 & $\begin{array}{l}\text { Physalis walteri } \\
\text { Nutt. }\end{array}$ & Solanaceae & groundcherry & leaves & $\begin{array}{l}\text { Physalis angulata } \\
\text { L. gonorrhea, } \\
\text { fever, Physalis } \\
\text { pubescens L. } \\
\text { stomachic }\end{array}$ \\
\hline ALA43 & Plantago major $\mathrm{L}$. & Plantaginaceae & $\begin{array}{l}\text { common } \\
\text { plantain }\end{array}$ & leaves & $\begin{array}{l}\text { wounds, boils, } \\
\text { dysentery, } \\
\text { diarrhea, colds }\end{array}$ \\
\hline ALA8 & $\begin{array}{l}\text { Psychotria } \\
\text { nervosa } \mathrm{Sw} .\end{array}$ & Rubiaceae & wild coffee & leaves & $\begin{array}{l}\text { colds, stomach } \\
\text { problems }\end{array}$ \\
\hline ALA44 & $\begin{array}{l}\text { Quercus } \\
\text { virginiana Mill. }\end{array}$ & Fagaceae & live oak & leaves & $\begin{array}{l}\text { febrifuge, } \\
\text { diarrhea, } \\
\text { antiseptic }\end{array}$ \\
\hline ALA11 & $\begin{array}{l}\text { Rhynchospora } \\
\text { colorata (L.) H. } \\
\text { Pfeiff. }\end{array}$ & Cyperaceae & $\begin{array}{l}\text { starrush, } \\
\text { whitetop }\end{array}$ & aerial & colds, bronchitis \\
\hline ALA25 & $\begin{array}{l}\text { Ricinus communis } \\
\text { L. }\end{array}$ & Euphorbiaceae & castorbean & leaves & $\begin{array}{l}\text { leaf decoction } \\
\text { wash for sores }\end{array}$ \\
\hline ALA27 & $\begin{array}{l}\text { Schefflera } \\
\text { actinophylla } \\
\text { (Endl.) Harms }\end{array}$ & Araliaceae & $\begin{array}{l}\text { Australian } \\
\text { umbrella tree }\end{array}$ & leaves & wound \\
\hline ALA28 & $\begin{array}{l}\text { Schinus } \\
\text { terebinthifolius } \\
\text { Raddi }\end{array}$ & Anacardiaceae & $\begin{array}{l}\text { Brazilian } \\
\text { pepper }\end{array}$ & leaves & $\begin{array}{l}\text { diarrhea, skin, } \\
\text { wound }\end{array}$ \\
\hline ALA45 & $\begin{array}{l}\text { Senna alata }(\mathrm{L} .) \\
\text { Roxb. }\end{array}$ & Fabaceae & $\begin{array}{l}\text { candlestick } \\
\text { plant }\end{array}$ & flowers, leaves & $\begin{array}{l}\text { diarrhea, } \\
\text { vermifuge, } \\
\text { venereal disease, } \\
\text { ringworm, } \\
\text { eczema, grippe, } \\
\text { catarrh }\end{array}$ \\
\hline ALA50 & $\begin{array}{l}\text { Stachytarpheta } \\
\text { jamaicensis (L.) } \\
\text { Vahl }\end{array}$ & Verbenaceae & $\begin{array}{l}\text { blue } \\
\text { porterweed, } \\
\text { joee }\end{array}$ & leaves & $\begin{array}{l}\text { boils, vermifuge, } \\
\text { coughs, fever, } \\
\text { skin ailments, } \\
\text { diarrhea, venereal } \\
\text { disease }\end{array}$ \\
\hline
\end{tabular}




\begin{tabular}{|c|c|c|c|c|c|}
\hline ALA46 & $\begin{array}{l}\text { Swietenia } \\
\text { mahagoni Jacq. }\end{array}$ & Meliaceae & $\begin{array}{l}\text { west Indian } \\
\text { mahogany }\end{array}$ & leaves & $\begin{array}{l}\text { diarrhea, } \\
\text { febrifuge, colds, } \\
\text { catarrh, venereal } \\
\text { disease }\end{array}$ \\
\hline ALA35 & $\begin{array}{l}\text { Tabebuia aurea } \\
\text { (Silva Manso) } \\
\text { Benth. \& Hook. f. } \\
\text { ex S. Moore }\end{array}$ & Bignoniaceae & $\begin{array}{l}\text { Caribbean } \\
\text { trumpettree }\end{array}$ & flowers, leaves & $\begin{array}{l}\text { Tabebuia } \\
\text { bahamensis } \\
\text { (Northr.) Britton } \\
\text { for gonorrhea, } \\
\text { Tabebuia rosea } \\
\text { (Bertol.) A.D.C. } \\
\text { fever, colds }\end{array}$ \\
\hline ALA2 & $\begin{array}{l}\text { Tetrazygia bicolor } \\
\text { (Mill.) Cogn. }\end{array}$ & Melastomataceae & $\begin{array}{l}\text { Florida } \\
\text { clover ash }\end{array}$ & leaves & night sweats \\
\hline ALA47 & $\begin{array}{l}\text { Tillandsia } \\
\text { recurvata (L.) L. }\end{array}$ & Bromeliaceae & ballmoss & whole plant & leucorrhea \\
\hline ALA9 & $\begin{array}{l}\text { Trema micrantha } \\
\text { (L.) Blume }\end{array}$ & Cannabaceae & nettletree & $\begin{array}{l}\text { leaves, stem, } \\
\text { fruits }\end{array}$ & skin eruptions \\
\hline ALA29 & $\begin{array}{l}\text { Vitis rotundifolia } \\
\text { Michx. }\end{array}$ & Vitaceae & muscadine & aerial & $\begin{array}{l}\text { Vitis caribaea } \\
\text { D.C. for venereal } \\
\text { disease, skin } \\
\text { condition, } \\
\text { diarrhea }\end{array}$ \\
\hline ALA65 & Annona glabra $\mathrm{L}$. & Annonaceae & pond apple & leaves, fruit & $\begin{array}{l}\text { diarrhea, burns, } \\
\text { cough, dysentery }\end{array}$ \\
\hline ALA66 & $\begin{array}{l}\text { Ficus citrifolia } \\
\text { Mill. }\end{array}$ & Moraceae & $\begin{array}{l}\text { wild banyan } \\
\text { tree }\end{array}$ & $\begin{array}{l}\text { leaves, latex, } \\
\text { fruits }\end{array}$ & wounds \\
\hline ALA64 & $\begin{array}{l}\text { Mangifera indica } \\
\text { L. }\end{array}$ & Anacardiaceae & mango & leaves & $\begin{array}{l}\text { cough, bronchitis, } \\
\text { asthma, fever, } \\
\text { diarrhea, sores, } \\
\text { skin disorders }\end{array}$ \\
\hline ALA67 & $\begin{array}{l}\text { Solanum } \\
\text { americanum Mill. }\end{array}$ & Solanaceae & $\begin{array}{l}\text { American } \\
\text { black } \\
\text { nightshade }\end{array}$ & leaves, fruits & $\begin{array}{l}\text { stomachic, } \\
\text { ringworm, } \\
\text { eyewash, } \\
\text { earwash, wounds, } \\
\text { abscess, sores }\end{array}$ \\
\hline
\end{tabular}

${ }^{a}$ Species nomenclature follows (Wunderlin \& Hansen, 2004). Family nomenclature follows (APGII, 2003). 


\subsubsection{Biomonitor organisms}

Chromobacterium violaceum: In C. violaceum (ATCC 12472), production of a purple pigment, violacein, is under QS-control (Lichstein \& van de Sand, 1945; Throup et al., 1995). This wild type strain produces and responds to the cognate autoinducer molecules C6-AHL and C4-AHL. The second biomonitor strain, CVO26, is a mutant of the wild type strain that is unable to produce its own AHL signal, but responds to exogenous active signal molecules (Cha, 1998). Anti-QS compounds inhibit production of violacein in both cases making these strains excellent for screening (McClean et al., 2004).

Agrobacterium tumefaciens NTL4 (pZLR4): A. tumefaciens strain NTL4 harboring plasmid pZLR4 (Luo et al., 2003) was used for the detection of both short (C6) and long-chain (C12) AHL suppression. In this system, the reporter gene (lacZ) is fused to a QS-dependant promoter. Activation of this promoter by exogenous AHLs causes concomitant expression of $\beta$-galactosidase, which produces a blue color by cleaving the chromophor from supplemented 5-bromo-4-chloro-3-indoyl- $\beta$-Dgalactopyranoside (X-gal). Anti-QS compounds inhibit the QS-dependant promoter and subsequent lacZ expression, thus limiting X-gal hydrolysis, and the appearance of blue color.

\subsubsection{Bioassays}

Fresh or dried plant material was ground using a mortar and pestle and placed directly onto Luria Bertani (LB) plates spread with either C. violaceum (wt), CV026 supplemented with AHL, or A. tumefaciens NTL4 supplemented with AHLs and X-gal. 
Plates were incubated overnight at $30{ }^{\circ} \mathrm{C}$, and QS inhibition was detected by a ring of colorless, but viable, cells around the disk. Purified halogenated furanone (100 $\mu \mathrm{g})$ was used as a positive control for QS inhibition, and ethanol $(20 \mu \mathrm{L})$ as a negative control. The ethanol was allowed to evaporate from the control and sample discs before testing to eliminate toxicity. A third control (tetracycline or gentamycin $10 \mu \mathrm{g}$ per disc) was included to compare antibiotic effect with anti-QS activity.

\subsubsection{Disk diffusion method}

Standard disc-diffusion assays were used to detect anti-QS activity of the plant extracts (Bauer et al., 1966). Each extract $(20 \mu \mathrm{L})$ was loaded onto sterile disks $(6 \mathrm{~mm}$ diameter) placed onto prepared LB plates spread with overnight culture $(100 \mu \mathrm{L})$ of $C$. violaceum wt, CV026, or A. tumefaciens NTL4. AHL $(30 \mu \mathrm{g})$ was added to both the CV026 and NTL4 plates, and $50 \mu \mathrm{L}$ of X-gal stock solution $(20 \mathrm{mg} / \mathrm{ml})$ was added to NTL4. Plates were incubated at $30{ }^{\circ} \mathrm{C}$ overnight and QS inhibition was detected by a ring of colorless but viable cells around the disk. Measurements were made from the outer edge of the disks to the edge of the zones of anti-QS inhibition. Controls were as previously described.

\subsection{Results}

\subsubsection{Preliminary screen to determine effect of surface microbes and processing}

To determine whether anti-QS compounds were actually from the plants themselves or from epiphyllic microorganisms, ethanol-washed plant material was compared with unwashed, placing whole or macerated leaves directly onto a prepared $C$. 
violaceum plate (Figure 2.1). No difference in anti-QS activity was observed in leaves without visible contamination (Figure 2.1) and only a slight increase in activity with leaves covered in epiphylls (data not shown). A second comparison between whole and macerated leaves revealed anti-QS activity only in crushed leaves (Figure 2.1). A third comparison between fresh and dried macerated leaves illustrated that in all cases tested both states retained anti-QS effect though in some cases it was reduced (data not shown).

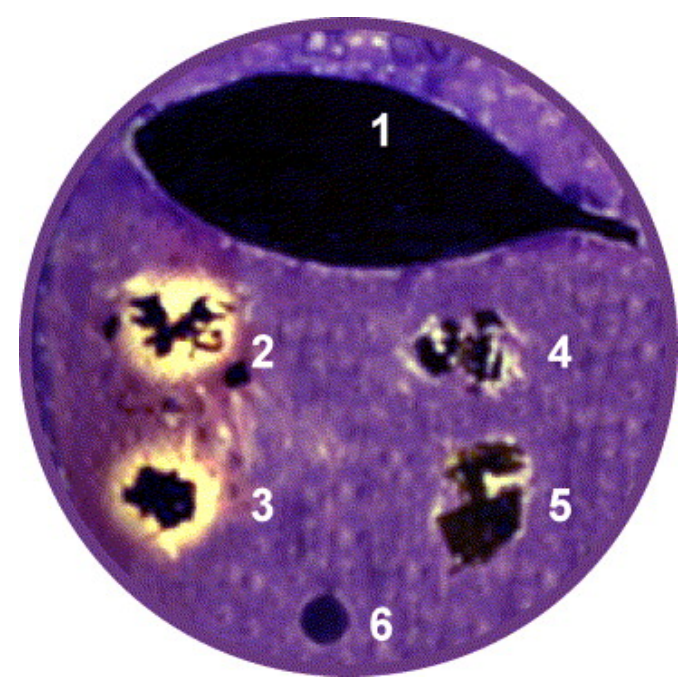

Figure 2.1 Preliminary screening of $C$. erectus for anti-QS activity using C. violaceum biomonitor strain. A yellowish "halo" of bacteria indicates an anti-QS effect. The samples were (1) washed whole leaf, (2) washed macerated leaves, and (3) unwashed macerated leaves of fresh C. erectus. As negative controls (4) washed, and (5) unwashed leaves of Hamelia patens and (6) sterile paper disk were included. 


\subsubsection{Anti-quorum sensing activities in southern Florida plants using $C$. violaceum assays}

Loss of purple pigment in C. violaceum is indicative of QS inhibition by the plant products introduced (Figure 2a). Out of 50 southern Florida plants (Table 2.1) screened for anti-QS activity, six species proved to be effective: C. erectus, $Q$. virginiana, $T$. bicolor, B. buceras, C. viminalis and C. hypericifolia (Table 2.2). The anti-QS activity of the six active species as fresh, dried, water-extracts, and ethanol-extracts were screened using the $C$. violaceum bioassays (Figure 2.2a and Table 2.2). Control discs containing halogenated furanone, gentamycin, and ethanol were included. As expected, a zone of growth inhibition was observed with gentamycin, a zone of QS inhibition (halo) was seen with the furanone, and no inhibition was apparent with ethanol (Figure 2.2a).

Strong anti-QS activity was observed in both the water and ethanol extracts of $C$. erectus, $C$. viminalis (inflorescence), B. buceras, and T. bicolor (Figure 2a and Table 2). For all species except $T$. bicolor and $C$. hypericifolia, the water extracts were more effective than those in ethanol. CV026 produced smaller halos overall, and no activity was detected with water extracts of $C$. viminalis and $Q$. virginiana leaves using this strain (Table 2).

\subsubsection{Anti-quorum sensing activities in southern Florida plants using}

\section{A. tumefaciens assays}

Loss of blue color in A. tumefaciens strain NTL4 (with added AHL) is indicative of QS inhibition by the plant products introduced as described in Materials and Methods. The six species, C. erectus, $Q$. virginiana, T. bicolor, B. buceras, $C$. viridis and $C$. 
hypericifolia, with anti-QS activity determined using the $C$. violaceum assay were then analyzed using an A. tumefaciens assay (Figure 2.2b and Table 2.2). Control discs containing halogenated furanone, tetracycline, and ethanol were included. As expected, a zone of growth inhibition was observed with tetracycline, a zone of QS inhibition was seen with the furanone, and no inhibition was apparent with ethanol (Figure 2.2a).
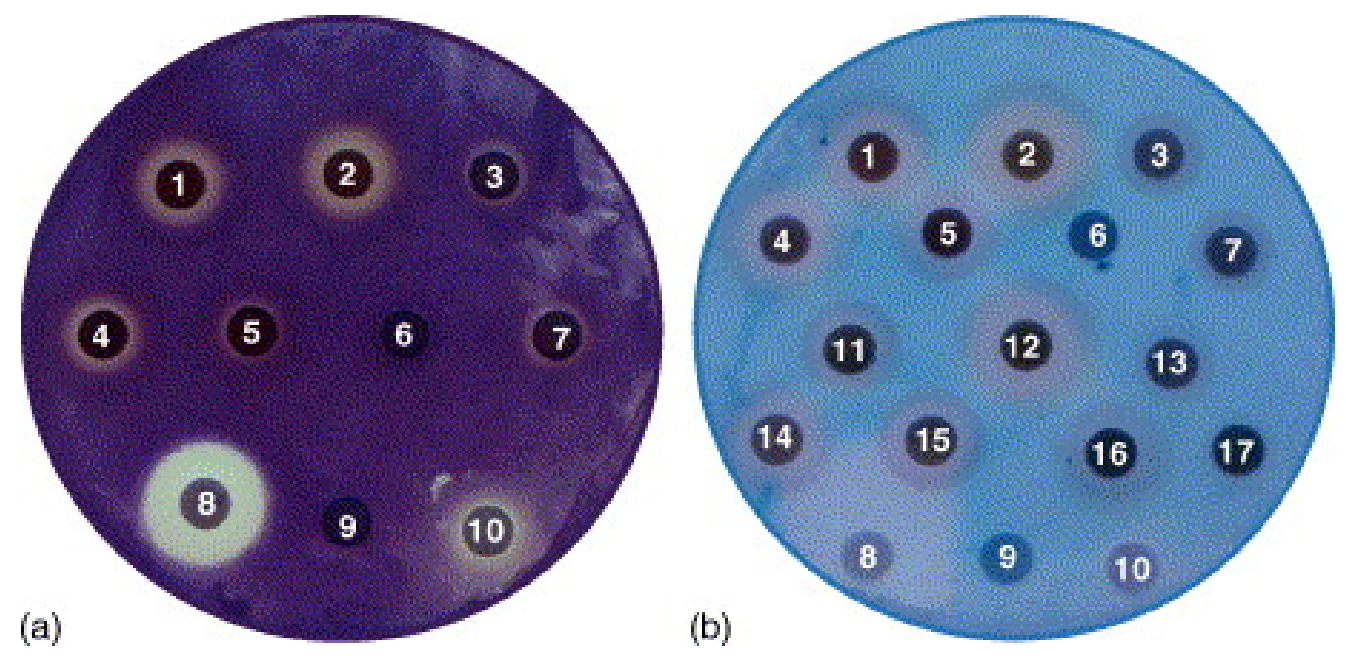

Figure 2.2 Anti-QS activity using (a) C. violaceum and (b) A. tumefaciens strains. AntiQS activity was tested using $20 \mu \mathrm{L}$ of water extracts of (1) C. erectus, (2) B. buceras, (3) C. viminalis-leaf, (4) C. viminalis-infloresence, (5) T. bicolor, (6) C. hypericifolia, and (7) Q. virginiana (7). Also included as controls were (8) $10 \mu \mathrm{g}$ gentamycin (a) and tetracycline (b), (9) $20 \mu \mathrm{l}$ ethanol, and (10) halogenated furanone. Discs 11 - 17 contain $20 \mu \mathrm{L}$ of ethanolic extracts of (11) C. erectus, (12) B. buceras, (13) C. viminalis-leaf, (14) C. viminalis-infloresence, (15) T. bicolor, (16) C. hypericifolia, and (17) $Q$. virginiana. 
Again, strong anti-QS activity was observed in both the water and ethanol extracts of $C$. erectus, C. viminalis (inflorescence), B. buceras, and T. bicolor (Figure 2.2b and Table 2.2). With $C$. erectus, $C$. viminalis (leaf), and $Q$. virginiana the water extracts were more effective than those in ethanol. Extracts of $B$. buceras and $C$. viminalis (inflorescence) produced similar-sized halos regardless of solvent, whereas T. bicolor and C. hypericifolia showed higher activity as ethanol extracts. None of the biomonitor strains detected any activity with a $C$. hypericifolia water extract (Table 2.2).

Table 2.2 Species showing anti-QS activity in this study

\begin{tabular}{|c|c|c|c|c|c|c|c|}
\hline \multirow[b]{2}{*}{ Active Plant Species } & \multirow[b]{2}{*}{ Part Tested } & \multirow[b]{2}{*}{ Solvent } & \multirow[b]{2}{*}{$\%$ Yield $^{b}$} & \multicolumn{4}{|c|}{ Zone of QS Inhibition $(\mathrm{mm})^{\mathrm{c}}$} \\
\hline & & & & CV & CVO26 & NTL4 C6 & NTL4 C12 \\
\hline \multirow[t]{2}{*}{ Conocarpus erectus $^{a}$} & Leaves & $\mathrm{H} 2 \mathrm{O}$ & 14.1 & 8.7 & 4.7 & 8.2 & 6.4 \\
\hline & & $\mathrm{EtOH}$ & 12.3 & 5.9 & 2.1 & 6.2 & 4.3 \\
\hline \multirow[t]{2}{*}{ Bucida burceras $^{a}$} & Leaves & $\mathrm{H} 2 \mathrm{O}$ & 12.9 & 8.3 & 5.4 & 8.6 & 8.9 \\
\hline & & $\mathrm{EtOH}$ & 13.9 & 8 & 3.2 & 8.4 & 9 \\
\hline \multirow[t]{2}{*}{ Callistemon viminalis } & Leaves & $\mathrm{H} 2 \mathrm{O}$ & 14.3 & 4.4 & 1.5 & 4.2 & 3.6 \\
\hline & & $\mathrm{EtOH}$ & 21 & 1.7 & 0 & 1.7 & 2 \\
\hline \multirow[t]{2}{*}{ Callistemon viminalis } & Infloresence & $\mathrm{H} 2 \mathrm{O}$ & 23.2 & 7 & 3.4 & 6.9 & 6.9 \\
\hline & & $\mathrm{EtOH}$ & 28 & 5.2 & 2.1 & 5.9 & 6.3 \\
\hline \multirow[t]{2}{*}{ Tetrazygia bicolor } & Leaves & $\mathrm{H} 2 \mathrm{O}$ & 22.5 & 5.7 & 2.2 & 5.8 & 5.4 \\
\hline & & $\mathrm{EtOH}$ & 16 & 6.2 & 2.2 & 6.4 & 5.5 \\
\hline \multirow[t]{2}{*}{ Chamaesyce hypericifolia } & Aerial & $\mathrm{H} 2 \mathrm{O}$ & 12.2 & 0 & 0 & 0 & 0 \\
\hline & & $\mathrm{EtOH}$ & 13.2 & 3.9 & 2.1 & 4.2 & 5.6 \\
\hline \multirow[t]{2}{*}{ Quercus virginiana } & Leaves & $\mathrm{H} 2 \mathrm{O}$ & 11.2 & 4 & 2 & 4.9 & 3.5 \\
\hline & & EtOH & 13.2 & 1.2 & 0 & 2.3 & 1.2 \\
\hline
\end{tabular}

${ }^{a}$ Species marked that showed slight antibacterial activity in addition to anti-QS.

${ }^{b}$ Percent yield and zone of QS inhibition for water $(\mathrm{H} 2 \mathrm{O})$ and ethanolic extracts $(\mathrm{EtOH})$.

c Average zones of QS inhibition using three biomonitor strains: Chromobacterium violaceum ATCC \#12472 (CV), C. violaceum CVO26 (CVO26), and Agrobacterium tumefaciens NTL4 with added C6-AHL (NTL4 C6) or C12-AHL (NTL4 C12). Average zones of killing for $10 \mu \mathrm{g} \mathrm{Gm}$ and $10 \mu \mathrm{g}$ Tc were 9.3 and $18.8 \mathrm{~mm}$, respectively. 


\subsubsection{Antibacterial vs. anti-quorum sensing activity}

The halos produced on lawns of the biomonitor strains could be the result of either (i) inhibition of cell growth or (ii) quenching of QS signals. Growth inhibition would produce a clear halo versus a turbid halo for the latter. To differentiate, the halo was examined under a higher magnification (Figure 2.3). As expected, the presence of the antibiotic gentamycin, created a clear zone of inhibition suggesting bactericidal activity. Under the same magnification, the area surrounding a disk containing crude extract of $C$. erectus showed normal cell growth.

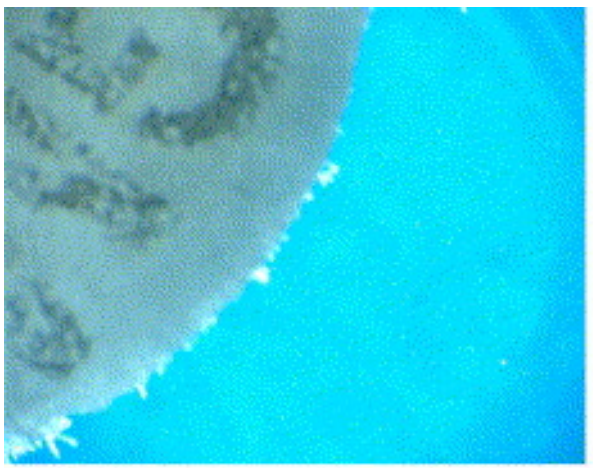

(a)

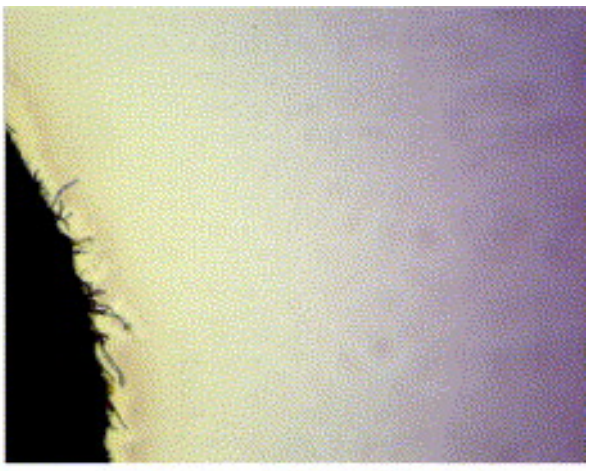

(b)

Figure 2.3 Antibacterial activity vs. anti-QS activity. Magnification of the areas surrounding (a) a gentamycin (Gm) disc, and (b) a disc containing ethanolic extract of $C$. erectus. The area around the disk in (a) is clear of cells, whereas in (b) there is a confluent layer of bacteria which lose their ability to produce violacein (anti-QS effect) in proximity to the disk.

The halo-effect is created by pigmentless (QS interrupted) cells adjacent to the disk, and the presence of violacein-producing (QS active) cells further out. In both $C$. 
violaceum and $A$. tumefaciens assays, C. erectus and B. burceras had antibacterial activity (zone of inhibition 1-2 mm) in addition to anti-QS activity (Figure 2.1). This activity was more pronounced with the $A$. tumefaciens biomonitor strain.

\subsection{Discussion and conclusions}

The aim of this study was to determine the anti-QS potential of southern Florida medicinal plants as a means to uncover a potential mode of action and to suggest a validation for continued traditional use. To date, research has shown anti-QS activity in only a few higher plants and seaweed (Fray, 2002; Gao et al., 2003; Manefield et al., 1999; Teplitski et al., 2000). This study introduces six medicinal plant species having anti-QS activity, representing five different families: C. erectus (Combretaceae), $Q$. virginiana (Fagaceae), B. buceras (Combretaceae), C. viminalis (Myrtaceae), T. bicolor (Melastomataceae), and C. hypericifolia (Euphorbiaceae) (Table 2.2). Our findings suggest that the anti-QS activity of plants on bacteria is perhaps more ubiquitous and diverse than previously thought.

Screening for anti-QS activity using multiple biomonitors (Table 2.2) eliminates artifact effects. If a plant showed activity with a $C$. violaceum strain and not $A$. tumefaciens, this activity might be limited to an aspect of violacein production downstream of the QS system. In contrast, if a sample had activity with A. tumefaciens and C12-AHL only, it might be limited to an effect on long chain signaling molecules. Since the six active species were effective at inhibiting QS in all biomonitor strains, we can assume that the responsible compounds have multiple or broad-spectrum effects. 
The preliminary screen provided some insight as to the effect of preparation on activity, something not often accounted for in medicinal plant studies. Leaves with excessive microbial colonization resulted in false-positive results. Therefore, care should be taken to eliminate bacterial and fungal causes of activity. An anti-QS effect was seen only in macerated leaves, suggesting that these compounds are not secreted on the leaf surface. Drying and extracting also affects efficacy in some cases, again indicating the importance of preparation. Hot water and ethanolic extracts were tested, as opposed to organic solvents, providing greater congruity with traditional preparation methods.

C. erectus, B. buceras, and T. bicolor have the highest anti-QS activities (Table 2.2). Commonly known as buttonwood, C. erectus is mainly used in southern Florida as an ornamental. The bark of this tree has been used for tanning leather and as a piscicide. Leaves are eaten or made into a decoction and taken for fever in parts of Africa and Puerto Rico (Burkhill, 1985; Irvine, 1961; Melendez, 1982). Buttonwood has also been used against catarrh, conjunctivitis, diarrhea, syphilis, and gonorrhea (Morton, 1981; Nellis, 1994). B. buceras is also used for syphilis and gonorrhea (Morton, 1981). A leaf decoction of T. bicolor is used in the Bahamas to treat "night sweats" (Morton, 1981), though it is unclear whether this ailment requires an analgesic or antibacterial treatment.

The water and ethanol extracts of $C$. viminalis inflorescences and the water extract from C. viminalis leaves have strong anti-QS activity (Table 2). The essential oil of C. viminalis has antihelminthic properties (Garg \& Kasera, 1982). Leaf decoctions, from species of the closely related genus Melaleuca, are used against a number of skin and respiratory conditions in the Bahamas, Puerto Rico, and Indochina (Duke, 1985; Melendez, 1982; Morton, 1981). Tea tree oil, M. alternifolia, has a long history of use in 
traditional medicine, and is a proven antimicrobial (Halcon \& Milkus, 2004; Hammer et al., 1996).

The water extract from $Q$. virginiana leaves exhibited more activity than its ethanol extracts. A bark decoction of $Q$. virginiana was used by Native Americans in the treatment of dysentery (Moerman, 1998). and in Cuba as a febrifuge and antiseptic (Morton, 1981). The extracts from Q. virginiana leaves and bark were applied externally, as an astringent analgesic to treat pain, sores, and hemorrhoids (Moerman, 1998).

Only the ethanol extracts of $C$. hypericifolia exhibited anti-QS activity, suggesting the active components are either significantly less polar than water, or that they are heat labile. In Guadalupe, C. hypericifolia is used to treat abrasions, opthalmia, diarrhea, respiratory ailments, and dysentery (Duke, 1985). It is also used in the Bahamas as a remedy for leucorrhea (Morton, 1981). A related species, C. hirta, has numerous medicinal uses in the Old World tropics and in the British Pharmacopoeia (Morton, 1981). It is used in Puerto Rico and many South American countries against fever, bronchitis, gonorrhea, and intestinal parasites (Bennett et al., 2002; Melendez, 1982).

Both $C$. erectus and B. buceras possess antibacterial as well as anti-QS activity. This poses the question of whether reduced concentrations of antibacterial compounds can create an anti-QS effect by reducing the cell count to a number below the quorum. Although it cannot be completely ruled out, in the case of two common antibiotics (tetracycline, gentamycin) this proved untrue. With both antibiotics, there was a distinct change from the zone of inhibition to viable cells. There was no loss of pigment in the cells immediately surrounding the kill zone, and in fact there was a ring of darker color. 
It is unclear at this stage whether the antibacterial and anti-QS effect in these plants are from the same or distinct chemical compounds.

Differential TLC migration suggests that more than one compound may contribute to anti-QS effect in these plants (data not shown). These compounds also seem distinct from those produced in bacteria and seaweed. To date the only known antiQS compounds from higher organisms are structural mimics (Manefield et al., 1999; Manefield et al., 2002). However, quorum quenching can occur through a number of other methods including signal binding, degradation, or direct interaction with QS genes (Smith \& Iglewski, 2003b). We are currently focusing on chemical identification of the anti-QS compounds in the screened efficacious extracts using chromatographic methods and mass spectrometry.

In summary, the anti-QS potential of medicinal plants may be as important as the antibacterial effect. In this study, we have shown six medicinal plants from southern Florida to have anti-QS activity. We propose that the anti-QS effect of the plants reported could contribute in part to their efficacy and traditional use as medicines. Most recent studies test only the antibacterial effect of plants used to treat infection leaving useful plant species to go unnoticed (e.g.: (Bonjar, 2004; Hernandez et al., 2003; Kloucek et al., 2005; Mothana \& Lindequist, 2005; Romero et al., 2005)). We suggest that other mechanisms of action, including anti-QS activity, should not be overlooked. Although biomonitor strains were chosen for ease of screening, similar QS systems exist in many human pathogens. Future work will focus on the effect of anti-QS compounds in these model organisms. 


\subsection{Acknowledgements}

We are thankful for the funds provided by the National Institute of Health (NIH), specifically the National Center for Complimentary and Alternative Medicine (NCCAM; 1-T32-AT01060-01) and Cystic Fibrosis Foundation Student Traineeship Grant (A.L.A). Also many thanks to Dr. Stephen Kjelleberg (The University of New South Wales, Sydney, Australia) for providing the halogenated furanones, Dr. Stephen K. Farrand (University of Illinois at Urbana- Champaign) for providing the biomonitor strain NTL4(pZLR4), and for all help provided by the Mathee and Bennett lab crews. 


\section{Chapter 3}

"Inhibition of quorum sensing-controlled virulence factor production in Pseudomonas aeruginosa by south Florida plant extracts"

As published in the Journal of Antimicrobial Agents and Chemotherapy

January 2008

Volume 52, Pages 198-203

Copyright (C) The American Society for Microbiology

Allison Adonizio, Kok-Fai Kong, Kalai Mathee 


\subsection{Abstract}

Quorum sensing (QS) is a key regulator of virulence and biofilm formation in Pseudomonas aeruginosa and other medically relavent bacteria. Aqueous extracts of six plants: Conocarpus erectus, Chamaesyce hypericifolia, Callistemon viminalis, Bucida buceras, Tetrazygia bicolor, and Quercus virginiana were examined in this study for their effects on P. aeruginosa virulence factors and the QS system. C. erectus, $B$. buceras, and $C$. viminalis cause significant inhibition of LasA protease, LasB elastase, pyoverdin production, and biofilm formation. Additionally, each plant presents a distinct profile of effect on the las and $r h l$ QS genes and their respective signaling molecules, suggesting different mechanisms responsible for efficacy. Extracts of all plants caused inhibition of QS genes and QS-controlled factors with marginal effects on bacterial growth, suggesting quorum quenching mechanisms unrelated to static or cidal effects.

Keywords: Quorum sensing, medicinal plants, virulence factor, Pseudomonas aeruginosa, LasA, LasB, pyoverdin, biofilm

\footnotetext{
Abbreviations: QS, Quorum Sensing; AHL, Acyl-Homoserine Lactone; OdDHL, N-(3oxododecanoyl)-L-homoserine lactone; BHL, N-butanoyl-L-homoserine lactone; AB, Agrobacterium Broth (minimum media); LB Luria-Bertani broth
} 


\subsection{Introduction}

Pneumonia due to microbial infections is a major cause of morbidity and mortality in immuno-compromised patients. Pseudomonas aeruginosa hails as the leading pathogen among patients suffering from cystic fibrosis, diffused pan-bronchitis, and chronic obstructive pulmonary disease (Hoiby, 1994; Lieberman, 2003; Registry, 2005). In addition, $P$. aeruginosa remains one of the major causes of nosocomial infections (Centers for Disease Control and Prevention, 2004). The success of this organism is largely due to the production of a myriad of virulence factors (including Las A protease, LasB elastase, pyoverdin, pyocyanin, and alginate) and its capability to form intractable biofilms (Lyczak et al., 2002).

Expression of many of the virulence factors in $P$. aeruginosa is controlled by the quorum sensing (QS) system (Venturi, 2006), an intercellular communication scheme in which bacteria are able to detect population density (via signaling molecules and receptors) and control gene expression accordingly (Schuster \& Greenberg, 2006). $\quad P$. aeruginosa elaborates two main sets of QS systems: lasI-lasR and rhlI-rhlR (Schuster \& Greenberg, 2006). LasI and RhlI are synthetases that manufacture the autoinducer signaling molecules $\mathrm{N}$-(3-oxododecanoyl)-L-homoserine lactone (OdDHL) and $\mathrm{N}$ butanoyl-L-homoserine lactone (BHL), respectively. These molecules diffuse out into the environment and, upon reaching a putative threshold concentration, activate receptors las $R$ and $r h l R$. These receptors, in turn, coordinate regulation of pathogenicity. A third signal, PQS (Pseudomonas Quinolone Signal), also plays an integral role in the QS system (Pesci et al., 1999). This secondary metabolite of $P$. aeruginosa is incorporated into the QS hierarchy in times of cell stress (McKnight et al., 2000). This intricate 
communication system of $P$. aeruginosa is mirrored in many gram-negative pathogenic bacteria, where it coordinates regulation of virulence, including motility, biofilm formation, and toxin production (De Kievit \& Iglewski, 2000; Donabedian, 2003; Fuqua \& Greenberg, 1998; Parsek \& Greenberg, 2000; Schauder \& Bassler, 2001).

The misuse and abuse of antibiotics in pharmacotherapy has led to the development of widespread resistance in the target organism. The failure of existing antibiotics to control infection makes it crucial to find alternatives to currently available drugs. Since pathogenicity in many bacteria is regulated by QS, inhibition of this system may cause attenuation of virulence and protect against infection (Hentzer \& Givskov, 2003; Juhas et al., 2005; Smith \& Iglewski, 2003a). In fact, an anti-QS approach has already shown promise in the battle against $P$. aeruginosa (Hentzer et al., 2003; Wu et al., 2004).

Anti-QS agents were first characterized in the red marine alga, Delisea pulchra, (Manefield et al., 1999; Manefield et al., 2002) and more recently, in a South Florida alga (Cumberbatch, 2002) and a few higher plants (Bjarnsholt et al., 2005; Gao et al., 2003; Teplitski et al., 2000). It has been shown that terrestrial plants not only produce autoinducer mimics to confound the bacterial QS system, but receive and respond to microbial signals as well (Adonizio et al., 2006; Bauer \& Mathesius, 2004). Given the promise of anti-QS compounds, efficient screening for these agents becomes imperative. In a previous study we utilized an ethnobotanically-directed search for anti-QS activity (Adonizio et al., 2006). We confirmed six South Florida medicinal plants: Conocarpus erectus, Chamaesyce hypericifolia, Callistemon viminalis, Bucida buceras, Tetrazygia bicolor, and Quercus virginiana to have anti-QS properties using Chromobacterium 
violaceum and Agrobacterium tumefaciens NTL4 biomonitor strains (Adonizio et al., 2006). These plants were chosen based on traditional use against respiratory and skin infections, conditions potentially caused or complicated by bacteria such as Pseudomonas aeruginosa.

Here we have taken this work a step further by exploring the effect of these six plants on the production of virulence factors and biofilms, AHL levels, and QS gene transcription in this organism. We demonstrate a significant decrease in the production of LasA protease, LasB elastase, pyoverdin, and biofilms in the presence of the extracts. Furthermore, each plant has a unique pattern of effect on the QS genes lasI/R and $r h l I / R$ and their respective signaling molecules OdDHL and BHL.

\subsection{Materials and methods}

\subsubsection{Plant extraction}

Conocarpus erectus (Combretaceae), Chamaesyce hypericifolia (Euphorbiaceae), Callistemon viminalis (Myrtaceae) (inflorescence), Bucida buceras (Combretaceae), Tetrazygia bicolor (Melastomataceae), and Quercus virginiana (Fagaceae), were collected and processed according to methods described previously (Adonizio et al., 2006). Briefly, pulverized plant material was extracted into boiling water, freeze-dried using a lyophilizer, and stored at $-20{ }^{\circ} \mathrm{C}$ until needed. Leaf extracts were tested unless otherwise noted. To complement the six active plants from the previous study, an additional plant with no anti-QS activity was chosen (Schefflera actinophylla, Apiaceae) as a negative control. 


\subsubsection{Strains and media}

Prototypic P. aeruginosa strain PAO1 (Holloway \& Morgan, 1986), and its derivatives (Kong et al., 2005) were used throughout this study. In addition, Staphylococcus aureus (ATCC \# 12600) was used in the LasA assay. Cells were maintained on Luria-Bertani broth (LB) plates and in LB liquid for overnight cultures. For quantitative assays, either LB (LasA assay only) or AB minimum media (added glucose and casein amino acids (20\% w/v) was used (Clark \& Maaloe, 1967).

\subsubsection{Culturing conditions}

For all assays except biofilm formation, culture conditions were as follows. Overnight cultures of PAO1 were grown in $\mathrm{LB}$ at $37{ }^{\circ} \mathrm{C}$ with shaking. Cultures were then diluted 100-fold into $\mathrm{AB}$ or LB media and allowed to grow to an $\mathrm{OD}_{600}$ of 1.7 (early stationary phase). At this point, the culture was divided into $10 \mathrm{ml}$ aliquots, and an additional $1 \mathrm{ml}$ of fresh media containing crude plant extract (or media control) was added to a final concentration of $1 \mathrm{mg} / \mathrm{ml}$ extract. Cultures were recovered at late stationary phase (approximately 12 hours after addition). Cells were separated from the growth media by centrifugation at $10,000 \times \mathrm{g}$ for 10 minutes.

\subsubsection{LasA staphylolytic assay}

LasA protease activity was determined by measuring the ability of culture supernatants to lyse boiled $S$. aureus cells (Kong et al., 2005). A $100 \mu$ l aliquot of $P$. aeruginosa LB culture supernatant with or without plant extracts was added to $900 \mu 1$ of 
boiled $S$. aureus suspension. $\mathrm{OD}_{600}$ was determined after $0,5,10,20,30,45$, and 60 minutes. Activity was expressed as $\Delta \mathrm{OD}_{600} /$ hour per $\mu \mathrm{g}$ protein.

\subsubsection{LasB elastolytic assay}

The elastolytic activity of $\mathrm{AB}$ culture supernatants was determined using elastin Congo red (ECR; Sigma, St. Louis, MO)(Ohman et al., 1980). A $100 \mu 1$ aliquot was added to $900 \mu \mathrm{l}$ of ECR buffer ( $100 \mathrm{mM}$ Tris, $1 \mathrm{mM} \mathrm{CaCl}$, $\mathrm{pH}$ 7.5) containing $20 \mathrm{mg}$ ECR. This was then incubated with shaking for 3 hours at $37{ }^{\circ} \mathrm{C}$. Insoluble ECR was removed by centrifugation, and the absorption of the supernatant was measured at 495 nm. Cell-free AB medium, alone, and with plant extracts were used as negative controls. Activity was expressed as $\Delta \mathrm{OD}_{495}$ per $\mu \mathrm{g}$ protein.

\subsubsection{Pyoverdin assay}

The pyoverdin assay was adapted from the methods of Cox and Adams (Cox \& Adams, 1985). The AB supernatant was diluted tenfold into Tris- $\mathrm{HCl}$ buffer ( $\mathrm{pH}$ 7.4), and $100 \mu \mathrm{L}$ aliquots were added to 96-well microtiter plates on ice. Relative concentration of pyoverdin was based on fluorescence of the supernatant at an excitation wavelength of $405 \mathrm{~nm}$ and an emission wavelength of $465 \mathrm{~nm}$ (Thurion Genious FluorSpec). Activity was expressed in relative fluorescence units (RFU). Although we consider pyoverdin to be a marker of QS, a drop in production may be due to an indirect effect via $\mathrm{pH}$ or iron concentration changes (Jacques et al., 2003). To eliminate the chance of false positives, iron concentration was tested using 1,10-phenanthroline and desferal (Yegorov et al., 1993), and solution $\mathrm{pH}$ was checked throughout the experiment. 


\subsubsection{Polyvinyl chloride (PVC) biofilm formation assay}

The effect of plant extracts on the attachment phase of biofilm formation was measured using the PVC biofilm formation assay (O'Toole \& Kolter, 1998). Briefly, overnight cultures of PAO1 were resuspended in fresh $\mathrm{AB}$ medium in the presence and absence of plant extracts. After a 10 hour incubation period at $30^{\circ} \mathrm{C}$, biofilms were visualized in the microtiter plates by staining with a crystal violet solution. Plates were rinsed to remove planktonic cells, and surface-attached cells were then quantified by solubilizing the dye in ethanol and measuring absorbance at $\mathrm{OD}_{650} \mathrm{~nm}$.

\subsubsection{AHL assay}

AHLs were extracted from AB culture supernatants with acidified ethyl acetate, dried under nitrogen, and quantified by electrospray mass spectroscopy (ESMS) after the methods of Makemson et al (Makemson et al., 2006). Peak intensities for BHL $(\mathrm{m} / \mathrm{z}=$ $172)$ and OdDHL $(\mathrm{m} / \mathrm{z}=298)$, and their sodium adducts $(\mathrm{m} / \mathrm{z}=194$ and 230 respectively) were combined and converted to concentration using a standard curve generated from the pure compounds. Background readings from samples extracted with alkaline ethyl acetate were subtracted from those of the acid-extracted bacterial cultures before conversion, as the lactone ring is broken by alkaline hydrolysis making AHLs too polar to be fully extracted into ethyl acetate.

\subsubsection{Growth curves}

The effect of plant extracts on cell proliferation was determined by monitoring of the PAO1 growth curve. Briefly, an overnight culture (LB) of PAO1 was diluted 100- 
fold into $1 \mathrm{~L} \mathrm{AB}$ or $\mathrm{LB}$ media. $\mathrm{OD}_{600}$ was monitored at 45 -minute intervals until an $\mathrm{OD}_{600}$ of $\sim 1.7$ (approximately 8 hours). The culture was then divided into $28 \mathrm{ml}$ aliquots, to which $2 \mathrm{ml}$ of $\mathrm{AB}$ or $\mathrm{LB}$ (controls) or $2 \mathrm{ml}$ concentrated extract were added. The final extract concentration was $1 \mathrm{mg} / \mathrm{ml}$. Cultures with added extract were normalized to the control $\mathrm{OD}_{600}$ of 1.7 at this time to account for plant pigmentation. Optical density was monitored at 1.5 hour intervals until a final time point of 24 hours. All OD $_{600}$ measurements were verified at $1 / 10$ dilution for greater accuracy.

\subsubsection{0 $\beta$-Galactosidase assay}

Transcriptional activity of QS-gene promoters was assayed using PAO1-derived strains harboring promoter-lac $Z$ fusions: $\mathrm{P}_{\text {lasi }}-l a c Z$ (pPCS223), $\mathrm{P}_{\text {lasR }}-l a c Z$ (pPCS1001), $\mathrm{P}_{\text {rhlI }}-l a c Z$ (pLPR1), $\mathrm{P}_{\mathrm{rhlR}}-l a c Z$ (pPCS1002), and as a control, a promoterless-lacZ fusion strain (pLP170) (Kong et al., 2005). Cultures were grown in AB media and monitored under the same conditions as PAO1, with extract added once the cultures reached an $\mathrm{OD}_{600}$ of 1.7. Assays for $\beta$-galactosidase activity in P. aeruginosa were performed using ONPG as previously described (Mathee et al., 1997).

\subsubsection{Bradford assay}

In addition to growth curve monitoring, the Bradford assay (Bradford, 1976) was performed to confirm that the reduction in virulence factors was not due to a decrease in cell density. Raw data from all assays were normalized to total protein concentration; however, there was no significant difference between sample sets. 


\subsubsection{Statistical analysis}

All experiments were performed independently in triplicate on pooled samples of biological replicates described in Adonizio et al 2006 (Adonizio et al., 2006). Data were analyzed using one-way analysis of variance (ANOVA) with a $P$-value of 0.05 using the statistical software package SPSS (Chicago, IL).

\subsection{Results3.4.1 LasA protease activity in the presence of plant extracts}

LasA staphylolytic protease is a $20-\mathrm{kDa}$ zinc metalloendopeptidase belonging to the $\beta$-lytic endopeptidase family of proteases (Kessler, 1995). There was a significant decrease in LasA activity when PAO1 was grown in the presence of B. buceras $(96 \%$ decrease), C. erectus (94\% decrease), T. bicolor (89\% decrease), C. viminalis (71\% decrease), or C. hypericifolia (49\% decrease) when compared to the control (Table 3.1). Addition of $Q$. virginiana, had no significant effect on LasA protease production. As expected, the negative control, S. actinophylla, also showed no significant change in Las A activity (Table 3.1).

\subsubsection{LasB elastase activity in the presence of plant extracts}

LasB elastase is a zinc metalloprotease capable of destroying or inactivating a wide range of biological tissues and immunological agents (Bever \& Iglewski, 1988). There was a significant decrease in LasB activity when PAO1 was grown in the presence of $C$. erectus ( $65 \%$ decrease) or C. viminalis $(63 \%$ decrease), when compared to the control. Growth of PAO1 with B. buceras, T. bicolor, or S. actinophylla (negative 
control) caused no significant effect, whereas $C$. hypericifolia, and $Q$. virginiana caused an increase in elastase activity.

Table 3.1 Effect of plant aqueous extracts on $P$. aeruginosa virulence factors

\begin{tabular}{lcccc}
\hline Culture conditions & LasA activity $^{\mathrm{a}}$ & Elastase activity $^{\mathrm{b}}$ & Pyoverdin production $^{\mathrm{c}}$ & Biofilm formation $^{\mathrm{d}}$ \\
\hline Media only & $0.274 \pm 0.016$ & $145.5 \pm 6.9$ & $4918 \pm 281$ & $0.64 \pm 0.01$ \\
C. erectus & $0.017 \pm 0.005^{*}$ & $48.2 \pm 11.8^{*}$ & $453 \pm 85^{*}$ & $0.38 \pm 0.19$ \\
B. buceras & $0.010 \pm 0.011^{*}$ & $130.8 \pm 31.5$ & $800 \pm 275^{*}$ & $0.14 \pm 0.01^{*}$ \\
C. viminalis & $0.080 \pm 0.004^{*}$ & $53.9 \pm 4.2^{*}$ & $1997 \pm 271^{*}$ & $0.07 \pm 0.01^{*}$ \\
T. bicolor & $0.030 \pm 0.015^{*}$ & $105.6 \pm 14.3$ & $2134 \pm 304^{*}$ & $0.13 \pm 0.02^{*}$ \\
Q. virginiana & $0.197 \pm 0.012$ & $499.1 \pm 36.0$ & $4423 \pm 422$ & $0.08 \pm 0.03^{*}$ \\
C. hypericifolia & $0.139 \pm 0.012^{*}$ & $553.6 \pm 19.4$ & $3068 \pm 295^{*}$ & $0.70 \pm 0.07$ \\
S. actinophylla & $0.224 \pm 0.014$ & $172.4 \pm 9.2$ & $4069 \pm 611$ & $0.53 \pm 0.16$ \\
\hline
\end{tabular}

${ }^{a}$ LasA activity was expressed as reduction in $\mathrm{OD}_{600}$ per hour per microgram total protein

${ }^{\mathrm{b}}$ Elastase activity was expressed as absorbance at $\mathrm{OD}_{495}$ per microgram of protein $* 1000$

${ }^{\mathrm{c}}$ Pyoverdin production was expressed as fluorescence at $465 \mathrm{~nm}$ (excitation $\lambda=405 \mathrm{~nm}$ ) per microgram of protein

${ }^{\mathrm{d}}$ Biofilm production was expressed as $\mathrm{OD}_{650}$ after incubation with crystal violet *Indicates significance at $p=0.05$ 


\subsubsection{Extracts alter pyoverdin production}

Pyoverdins are virulence factors in that they compete with mammalian transferrin for iron, the successful sequestration of which essentially starves the host tissues (Meyer et al., 1996). They also promote pathogenicity by stimulating bacterial growth (Cox \& Adams, 1985). One of the pyoverdins is suggested to be a QS-like molecule, regulating both itself and production of other toxins (Beare et al., 2003; Lamont et al., 2002). All plant extracts with the exception of $Q$. virginiana and $S$. actinophylla (negative control), showed a significant reduction of pyoverdin production; however, C. hypericifolia was only marginally significant (Table 3.1 ). The most active extracts were $C$. erectus and $B$. buceras, with a substantial decrease in pyoverdin activity (91\% decrease and 84\% decrease respectively) when compared to the control (Table 3.1). All of the culture supernatants retained a $\mathrm{pH}$ of $\sim 7.0$ regardless of amount or type of extract added. Although statistically significant differences were found between iron concentration in the extracts, there was no trend which could be correlated with activity on pyoverdin (data not shown).

\subsubsection{Plant extracts have an inhibitory effect on biofilm formation}

P. aeruginosa has the ability to form biofilms, a partially QS controlled phenomenon (Davies et al., 1998), where cells are organized into layers, and enmeshed in a matrix of mucoid polysaccharides (Costerton, 1995). A switch to the biofilm mode of growth confers increased antibacterial resistance and creates a considerably more severe infection in the cystic fibrosis lung (Lyczak et al., 2002). There was a significant decrease in biofilm formation when PAO1 was grown in the presence of $C$. viminalis 
( $89 \%$ decrease), Q. virginiana ( $88 \%$ decrease), T. bicolor ( $80 \%$ decrease), or B. buceras ( $78 \%$ decrease) when compared to the control (Table 3.1). C. erectus caused a $41 \%$ decrease in biofilm formation; however, this result was marginally insignificant.

\subsubsection{Plant extracts affect QS gene expression}

With the exception of $Q$. virginiana, C. hypericifolia, and the negative plant control S. actinifolia, most extracts had a significant effect on QS gene expression (Table 3.2). The most significant decreases in lasI expression were found with $C$. viminalis ( $80 \%$ decrease), B. buceras ( $49 \%$ decrease), T. bicolor (41\% decrease), and C. erectus (38\% decrease). C. erectus and C. viminalis show the greatest reduction of lasR expression (56\% and 48\% decrease, respectively). Whereas T. bicolor ( $42 \%$ decrease), B. buceras (55\% decrease), and C. erectus ( $40 \%$ decrease) show the greatest reductions in rhlI expression. These same three plants also affect the greatest reductions in rhlR expression $(66 \%, 62 \%$, and $66 \%$ decrease respectively).

\subsubsection{Plant extracts affect the production of AHL molecules}

Notable decreases in OdDHL levels were seen with C. viminalis (46\% reduction from the control) and B. buceras (38\% reduction). Significant decreases in BHL levels corresponded to the addition of B. buceras, T. bicolor, and C. hypericifolia extract, with reductions of $(41 \%, 39 \%$, and $35 \%$, respectively) (Table 3.2). 
Table 3.2 Effect of plant extracts on $P$. aeruginosa QS genes and AHL production

\begin{tabular}{|c|c|c|c|c|c|c|}
\hline \multirow{2}{*}{ Culture conditions } & \multicolumn{2}{|c|}{ AHL Production $^{\mathrm{a}}$} & \multicolumn{4}{|c|}{ Gene Expression ${ }^{b}$} \\
\hline & C12-AHL & C4-AHL & lasI & las $R$ & rhlI & rhlR \\
\hline Media only & $1.216 \pm 0.19$ & $0.789 \pm 0.10$ & $3363 \pm 311$ & $5008 \pm 256$ & $4855 \pm 459$ & $8271 \pm 655$ \\
\hline C. erectus & $0.981 \pm 0.14$ & $0.597 \pm 0.01$ & $2101 \pm 270^{*}$ & $1489 \pm 102 *$ & $2917 \pm 265^{*}$ & $3004 \pm 406^{*}$ \\
\hline B. buceras & $0.751 \pm 0.16^{\mathrm{x}}$ & $0.468 \pm 0.12^{*}$ & $1718 \pm 147^{*}$ & $2377 \pm 179^{*}$ & $2200 \pm 249^{*}$ & $3339 \pm 440 *$ \\
\hline C. viminalis & $0.659 \pm 0.15^{*}$ & $0.533 \pm 0.02 *$ & $662 \pm 86^{*}$ & $1745 \pm 182^{*}$ & $3370 \pm 300^{*}$ & $3751 \pm 120^{*}$ \\
\hline T. bicolor & $1.501 \pm 0.29$ & $0.483 \pm 0.03 *$ & $2000 \pm 183^{*}$ & $2038 \pm 202^{*}$ & $2810 \pm 449 *$ & $2930 \pm 282^{*}$ \\
\hline Q. virginiana & $1.041 \pm 0.19$ & $0.586 \pm 0.09$ & $2806 \pm 204$ & $3374 \pm 460^{*}$ & $3128 \pm 299 *$ & $5324 \pm 306^{*}$ \\
\hline C. hypericifolia & $0.892 \pm 0.02$ & $0.511 \pm 0.08^{*}$ & $2765 \pm 249$ & $3324 \pm 97^{*}$ & $3433 \pm 412 *$ & $3635 \pm 319^{*}$ \\
\hline S. actinophylla & $1.146 \pm 0.17$ & $0.761 \pm 0.10$ & $3715 \pm 363$ & $4692 \pm 200$ & $4748 \pm 181$ & $7347 \pm 628$ \\
\hline
\end{tabular}

${ }^{\mathrm{a}} \mathrm{AHL}$ production was expressed in $\mu \mathrm{M}$

${ }^{\mathrm{b}}$ Gene expression was measured via $\beta$-galactosidase activity of the lac $Z$ gene fusion products and expressed as Miller Units

*Indicates significance at $p=0.05 \quad{ }^{\mathrm{x}}$ Indicates significance at $p=0.10$

\subsubsection{Extracts have minimal effect on PAO1 growth after log phase}

Addition of extract at early stationary phase was chosen to limit any confounding effects on growth. When extracts were added at the beginning of the cell cycle (time zero), there were changes (slight delay or acceleration) in the logarithmic phase (data not shown). This did not, however, impact the endpoint cell density in most cases. To confirm an anti-QS mode of action, rather than logarithmic changes, a growth curve was 
taken controlling for the latter (Figure 3.1). Cultures of PAO1 were grown to early stationary phase before addition of compound (Figure 3.1, arrow). This insures that all samples would have the same opportunity (length of time and cell density) to reach the point of QS-controlled production of virulence factors. Stationary phase was reached in all samples (including control) approximately 8 hours after extract addition. Addition of extract did not significantly affect cell density or total protein.

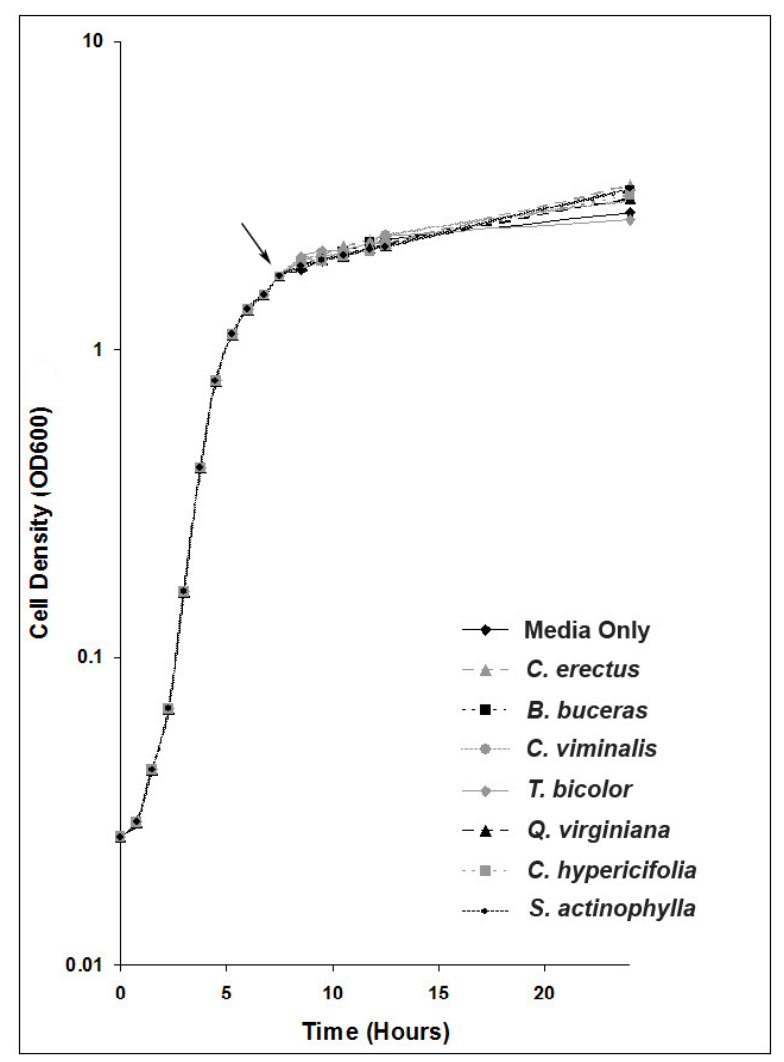

Figure 3.1 Influence of medicinal plant extracts on growth of $P$. aeruginosa (semi-log graph). Extracts were added during early stationary phase (approximately 8 hours) indicated by arrow. The data represent mean values of experiments performed in triplicate. 
The majority of the samples have little effect on the growth curve of PAO1 after $\log$ phase (Figure 3.1). The growth curve with added T. bicolor closely follows that of the media-only control, whereas $Q$. virginiana, B. buceras, C. hypericifolia, $Q$. virginiana, C. erectus and S. actinophylla all exhibit a slight increase in cell density (Figure 3.1). This increase is not significant, and is possibly due to added nutrients. However, it verifies that the anti-QS effect of these plants is not due to cell death.

Incidentally, addition of $T$. bicolor and $Q$. virginiana at time zero resulted in cell densities below the control (data not shown). This suggests an additional antibacterial effect of these plant extracts. However, total protein (measured via Bradford assay) of these samples did not differ significantly from the control.

\subsection{Discussion}

Six South Florida medicinal plants, C. erectus (Combretaceae), C. hypericifolia (Euphorbiaceae), C. viminalis (Myrtaceae), B. buceras (Combretaceae), T. bicolor (Melastomataceae), and Q. virginiana (Fagaceae), were examined for their anti-QS activity against $P$. aeruginosa PAO1. The virulence of $P$. aeruginosa is owed to its capacity to degrade host tissue with proteases and toxins, and to evade antibiotic attack by forming biofilms. Biofilm formation and the virulence factors examined in this study are under QS control (Davies et al., 1998; Kharazmi, 1989; Meyer et al., 1996; Morihara \& Homma, 1985). Thus, the plant extracts were examined for their ability to interfere with QS-dependent production of $P$. aeruginosa virulence factors LasA, LasB, and pyoverdin. In addition, we examined the ability of the extracts to inhibit biofilm formation, QS gene expression, and AHL synthesis. 


\subsubsection{Plant extracts differentially affect biofilm formation}

Since QS is involved in biofilm formation (Davies et al., 1998), we expected the plants posessing anti-QS activity to have a significant effect. Indeed, all extracts tested affected a decrease in biofilm formation, with the exception of $C$. hypericifolia. Interestingly, $Q$. virginiana exhibited an effect on biofilm production, though no other virulence factors, suggesting either a physical inhibition of biofilm growth or repression of biofilm genes and components outside the QS system. Disruption of the QS system using furanones has also been shown to inhibit biofilm growth (Hentzer et al., 2002). Previous work with garlic and D. pulchra furanones showed a qualitative change in biofilm morphology and a reduction in thickness; however, these analyses were not quantified (Hentzer et al., 2002; Hentzer et al., 2003; Rasmussen et al., 2005).

\subsubsection{Plant extracts differentially affect production of virulence factors}

P. aeruginosa proteases LasA and LasB are believed to play a major role in pathogenesis via host tissue degradation (Kharazmi, 1989; Morihara \& Homma, 1985). With the exception of $Q$. virginiana, all other plant species resulted in a significant decrease in the LasA activity of $P$. aeruginosa, with the most drastic reductions seen in $B$. buceras, C. erectus, and T. bicolor. No prior studies have considered LasA activity in the presence of anti-QS compounds.

The LasB elastase activity was significantly reduced in the presence of C. erectus and C. viminalis. Extracts from B. buceras and T. bicolor had no significant effect, whereas $C$. hypericifolia and $Q$. virginiana caused an increase in elastase activity. It is likely that the compounds in the latter two plants may upregulate the production of LasB 
and/or enhance the elastase activity. The compounds are not likely to be elastase-like proteins in the plants since the extraction process would have denatured most proteins. In comparison, recent studies on garlic (at $2 \% \mathrm{v} / \mathrm{v}$ ) show a $50 \%$ decrease in LasB activity (Rasmussen et al., 2005), whereas purified halogenated furanone from D. pulchra (10 $\mu \mathrm{M})$ induces an approximate $90 \%$ decrease (Hentzer et al., 2003).

All plant extracts, with the exception of $Q$. virginiana, caused a significant reduction of pyoverdin production. Mixed results on pyoverdin production have been previously observed with furanones. A naturally-occurring furanone from D. pulchra ((5Z)-4-bromo-5-(bromomethylene)-3-butyl-2(5H)-furanone) actually increases production of pyoverdin (Sakar et al., 2005). Whereas furanone C-30, a synthetic derivative of a compound from $D$. pulchra, confers a $90 \%$ reduction in pyoverdin levels (Hentzer et al., 2003). Neither iron-limitation nor $\mathrm{pH}$ is sufficient to explain the effect on virulence factor production. There were also no significant changes in cell growth corresponding to pyoverdin production, leaving anti-QS effect as the most plausible hypothesis.

\subsubsection{Mechanistic musings: multiple targets or global effect.}

Quorum inhibition occurs via a number of different mechanisms, the most well known being signal mimicry, such as in the case of the furanones (Manefield et al., 1999; Manefield et al., 2002). Other methods include signal degradation by proteins such as lactonase or acylase (Dong et al., 2001), signal binding, inhibition of genetic regulation systems, or interruption of downstream virulence and biofilm genes (for a review see (Whitehead et al., 2001b)). 
The current view of the $P$. aeruginosa QS hierarchy suggests that las controls $r h l$ with virulence proteins at the bottom of the ladder (Schuster \& Greenberg, 2006). The virulence factors LasA (staphylolytic protease) and LasB (elastase) are generally thought to be under control of the lasI/R system (Gambello, 1991; Schuster \& Greenberg, 2006), however $r h l I / R$ also controls activity to a lesser extent (Brint \& Ohman, 1995; Pearson et al., 1997). Pyoverdin is believed to be under rhlI/R control (Brint \& Ohman, 1995; Whiteley, 1999), whereas biofilm production is only partially under QS control (Davies et al., 1998). The redundant and auto-regulatory nature of the QS system is quite convoluted (Van Delden \& Iglewski, 1998). This, compounded with the complex phytochemistry of plant extracts prevents us from precisely linking QS gene expression, AHL level, and virulence factor production.

Although the mechanism of action of these extracts is a complex problem, there is an overall inhibition of the QS system with each of the tested plant extracts (with the exception of the control S. actinifolia). This somewhat general effect points to one of two explanations. The first, suggests that multiple chemicals in the plants may be causing distinct effects on different aspects of the QS system. The second explanation is that the effect is not directly on the las/rhl system, but rather on a more global QS regulator such as Vfr (Albus et al., 1997) or GacA (Reimmann et al., 1997).

There is also a trend in which the plant species that have less of an effect on AHL production and las/rhl expression also have less activity on $P$. aeruginosa virulence factors. This is most clearly seen with $Q$. virginiana, C. hypericifolia, and the control, $S$. actinifolia (Tables 3.1 and 3.2). Although not absolute, the converse is generally true. 
Overall, the most significant effects on the QS system were found with C. viminalis, $B$. buceras, and C. erectus.

Distinct patterns of thin layer chromatography (TLC) migration in these three extracts (data not shown) suggest that they contain multiple active compounds, perhaps functioning with separate mechanisms. At this point we do not have sufficient data to pinpoint the method of quorum quenching, we will therefore withhold further mechanistic speculation until these compounds are purified from the plants.

\subsection{Conclusions}

In summary, this work presents six plant species, representing five distinct families, which have a differential but significant effect on virulence factors, biofilms, QS gene expression, and signal production at a concentration of $1 \mathrm{mg} / \mathrm{ml}$. This concentration, although high, is relevant to traditional medicinal use in teas and poultices. More significantly, this concentration represents a crude aqueous extract and therefore should be much lower as a putative anti-QS compound. Concentrations were tested down to $0.25 \mathrm{mg} / \mathrm{ml}$, and some extracts, notably that of $C$. erectus, still had an effect on virulence factor production (unpublished results).

None of the plant extracts tested had a significant effect on the growth of PAO1 when added at early stationary phase (Figure 3.1, arrow). However, addition of $T$. bicolor and Q. virginiana at time zero resulted in cell densities below the control (data not shown), which suggests an additional antibacterial effect of these plants. All other plant extracts significantly reduced one to four QS-controlled virulence factors, genes, 
and AHL levels without a reduction in growth. This would strongly suggest an anti-QS rather than an antibacterial effect.

The top candidates for further anti-QS investigation are C. erectus, B. buceras, and $C$. viminalis. C. erectus significantly decreases LasA, LasB, and pyoverdin production, but not biofilm formation, whereas $B$. buceras affects all but elastase activity. B. buceras also has a significant effect on the expression of lasI, lasR, rhlI, and rhlR, and the concentration of OdDHL and BHL. C. erectus exhibits the same pattern of effect on QS genes and signaling molecules, though to a lesser extent. C. viminalis decreases all three virulence factors and biofilm formation, all QS genes tested and OdDHL. Research is currently underway in our lab to isolate active chemicals from these species.

The plant kingdom has long been a source of medicines, and as such, there have been many ethnobotanically-directed searches for agents to treat infections (e.g.: (Camporese et al., 2003; Cowan, 1999; Gnanamani et al., 2003; Hernandez et al., 2003)). However, most studies focus solely on bactericidal effects. Since the plants in this study show little, if any, cidal activity (Adonizio et al., 2006), quorum inhibition remains a potential mode of action. Shifting our focus to anti-QS and anti-virulence may reveal new quorum quenching compounds from medicinal plants and provide a novel method of treating infection.

In conclusion, the effects of the studied plant extracts on P. aeruginosa are quite complicated, and perhaps extend beyond the domain of the QS hypothesis. However, the reduction of QS gene expression and signaling molecules and the end-effect on virulence factor production provides some insight into why these plants were used in the past and how they can be used in the future to combat $P$. aeruginosa and other bacterial infections. 


\subsection{Acknowledgments}

We gratefully acknowledge the support of National Institute of Health, National Center for Alternative and Complementary Medicine NRSA \#1-T32-AT01060-01 (AA, KM), the Cystic Fibrosis Foundation Traineeship Grant \#ADONIZ06H0 (AA), the NIGMS-RISE program \#R25 GM61347 (AA), Florida International University Teaching Assistantship (KFK), Dr. John Makemson, members of the Mathee lab for assistance and support (especially Melissa Doud and Lance Umansky), and the Roy lab for gracious use of the fluorescence spectrophotometer. We would also like to thank the reviewers for their critical reading of this manuscript and their invaluable suggestions and comments. 


\section{Chapter 4}

"Attenuation of Pseudomonas aeruginosa virulence by medicinal plants in a Caenorhabditis elegans model system"

Accepted for publication by the Journal of Medical Microbiology

February 2008

Copyright (C) Society for General Microbiology.

Allison Adonizio, Sixto M. Leal Jr, Frederick M. Ausubel, Kalai Mathee 


\subsection{Abstract}

Expression of a myriad of virulence factors and innate antibiotic resistance enables the opportunistic human pathogen Pseudomonas aeruginosa to create intractable infections. Using a nematode model we efficiently screened for novel inhibitors of this

pathogen. Aqueous extracts of three plants: Conocarpus erectus, Callistemon viminalis, and Bucida buceras were examined in this study for their effects on P. aeruginosa killing of the nematode Caenorhabditis elegans. Results were evaluated in both toxin-based and infection-based assays with $P$. aeruginosa strains PAO1 and PA14. The tested plant extracts prevented mortality via gut infection in almost $60 \%$ of the worms, and caused a $50-90 \%$ reduction in death from toxin production. All extracts inhibited nematode death by $P$. aeruginosa without host toxicity, indicating their potential for further development as anti-infectives.

Keywords: Quorum sensing, medicinal plants, virulence factors, Pseudomonas aeruginosa, Caenorhabditis elegans, nematode

Abbreviations: QS, Quorum Sensing; AHL, Acyl-Homoserine Lactone; WT, Wild Type; BHI, Brain-Heart Infusion media; NGM, Nematode Growth Media 


\subsection{Introduction}

Pseudomonas aeruginosa is one of the leading pathogens among patients suffering from cystic fibrosis, diffused pan-bronchitis, and chronic obstructive pulmonary disease (Hoiby, 1994; Lieberman, 2003; Registry, 2005). In addition, it remains one of the major causes of nosocomial infections (Centers for Disease Control and Prevention, 2004). The success of this organism is attributed to numerous virulence factors (Smith \& Iglewski, 2003a; Tang et al., 1996), its ability to form biofilms (Costerton, 1995) and innate antibiotic resistance (De Kievit et al., 2001; Fisher et al., 2005).

Current anti-pseudomonal treatment includes elevated doses of beta-lactam, fluoroquinolone, or aminoglycoside antibiotics (Hauser \& Sriram, 2005). However, these drugs possess a high degree of toxicity, and mucoid strains of $P$. aeruginosa are rarely eradicated by these treatments (Hauser \& Sriram, 2005; Pederson, 1992). The failure of existing antibiotics to control infection makes it crucial to find alternatives to currently available drugs. Since pathogenicity in many bacteria is regulated by quorum sensing (QS), or cell-to-cell communication, inhibition of this system can cause attenuation of virulence and protect against infection (Hentzer et al., 2003; Juhas et al., 2005; Smith \& Iglewski, 2003b).

Plants have evolved numerous chemical strategies for deterring pathogen attack, including the production of bactericidal and anti-infective compounds, leading to their use as medicines [for a review see (Lewis \& Ausubel, 2006)]. In our previous work, we demonstrated that a number of medicinal plants exhibit anti-QS activity (Adonizio et al., 2006). Extracts of these plants were later shown to have an effect on virulence factor production, biofilm formation, QS-gene expression, and autoinducer production in $P$. 
aeruginosa (Adonizio et al., 2008b). In this study, we assessed the ability of three plant extracts to attenuate $P$. aeruginosa killing of the nematode, Caenorhabditis elegans.

C. elegans is well established as a pertinent and practical model for studying bacterial virulence (Darby et al., 1999; Tan \& Ausubel, 2000), as a number of $P$. aeruginosa factors important in the killing of $C$. elegans are also relevant to mammalian systems (Rahme et al., 1995; Tan et al., 1999a). "Fast-killing" of C. elegans by $P$. aeruginosa strain PA14 (on rich media) is mediated through production of virulence factors such as phenazines, whereas "slow-killing" (on minimal media) occurs via ingestion of the bacteria and subsequent infection (Mahajan-Miklos et al., 1999; Tan et al., 1999a; Tan et al., 1999b). The related strain PAO1 causes death through cyanide poisoning and neuromuscular paralysis (Gallagher \& Manoil, 2001). Importantly, $P$. aeruginosa-mediated killing of C. elegans in all three of these cases is dependent in part on the QS system (previous references). Thus, addition of plant compounds that have an effect on $P$. aeruginosa $\mathrm{QS}$, should therefore attenuate virulence factor production and subsequent death of C. elegans.

The advantage in using a live animal model when screening for anti-infective compounds is that both efficacy and host toxicity of a plant extract can be tested concurrently. In this report, we show that extracts from three different plant species cause a marked decrease in $P$. aeruginosa killing of $C$. elegans, without affecting worm fitness on E. coli. This approach can be expanded to the screening of natural product libraries or native extract sources. 


\subsection{Methods}

\subsubsection{Preparation of plant extracts}

Conocarpus erectus (Combretaceae), Callistemon viminalis (Myrtaceae), and Bucida buceras (Combretaceae) were collected and processed according to methods described previously (Adonizio et al., 2006). Briefly, pulverized plant material was extracted into boiling water, freeze-dried using a lyophilizer, and stored at $-20{ }^{\circ} \mathrm{C}$ until needed. Lyophilized extracts were reconstituted in a small volume of sterile water and added to the molten agar at a final concentration of $1 \mathrm{mg} \mathrm{ml}^{-1}$.

\subsubsection{Bacterial strains and growth conditions}

Because the tested extracts have been shown to affect the bacterial quorum sensing (QS) system (Adonizio et al., 2008b; Adonizio et al., 2006) the QS-mutant $\Delta$ lasR was used as a reference strain. P. aeruginosa PAO1 (Holloway \& Morgan, 1986) and its isogenic mutant PAO1DlasR (Gambello, 1991) were used in the paralytic assay. PA14 (Rahme et al., 1995) and PA14_lasR (Liberati et al., 2006) were used in the slow killing and fast killing assays. Escherichia coli OP50 was used as the control in all assays as it is the standard laboratory food of C. elegans (Brenner, 1974). All bacterial strains were grown overnight in LB broth at $37^{\circ} \mathrm{C}$, and transferred to plates depending on the required conditions.

\subsubsection{Nematode culture}

The wild type $C$. elegans (Bristol) N2 hermaphrodite strain was used in this study (Brenner, 1974). Worms were synchronized by hypochlorite treatment of gravid adults, 
hatching the eggs overnight in M9 minimum buffer (Brenner, 1974), and plating L1-stage worms onto lawns of E. coli on nematode growth media (NGM) plates (Brenner, 1974). Synchronized worms were grown to L4 or young adult stage at $25{ }^{\circ} \mathrm{C}$ for use in the killing assays.

\subsubsection{C. elegans paralytic assay (PAO1)}

Brain-heart infusion (BHI) agar plates with or without plant extract were seeded with $10 \mu \mathrm{l}$ of an overnight culture of OP50, PAO1 or PAO1 $\Delta$ lasR, and incubated at $37^{\circ} \mathrm{C}$ for 24 hours to form lawns of bacteria (Darby et al., 1999). Nematodes were washed off stock plates and suspended in a minimal volume of M9 buffer ( $\mathrm{pH}$ 6.5). Droplets containing 20-40 adult nematodes were placed onto the $P$. aeruginosa lawns and the plates were then incubated at room temperature $\left(21-23^{\circ} \mathrm{C}\right)$. Worms were evaluated for viability every hour for a total of 4 hours. Worms were scored as dead when they no longer responded to physical stimuli.

\subsubsection{C. elegans fast killing assay (PA14)}

Fast killing plates (peptone-glucose medium with $0.15 \mathrm{M}$ sorbitol) (MahajanMiklos et al., 1999) with or without plant extract were seeded with $10 \mu \mathrm{l}$ of an overnight culture of OP50, PA14 wt, or PA14_lasR. Plates were incubated for 24 hours at $37^{\circ} \mathrm{C}$, then at room temperature $\left(\sim 21-23^{\circ} \mathrm{C}\right)$ for another $12 \mathrm{~h}$. Approximately $20 \mathrm{~L} 4$ stage $C$. elegans were transferred with a wire pick onto plates at this time. Worms were evaluated for viability every hour for a total of 4 hours. As in the previous assay, worms were considered dead when they no longer responded to physical stimuli. 


\subsubsection{C. elegans slow killing assay (PA14)}

Slow killing plates (modified NGM) (Tan et al., 1999b) with or without plant extract were seeded with $10 \mu \mathrm{l}$ of an overnight culture of OP50, PA14 wt, or PA14 1 lasR. Plates were incubated for $24 \mathrm{~h}$ at $37^{\circ} \mathrm{C}$, then at room temperature $\left(\sim 21-23{ }^{\circ} \mathrm{C}\right)$ for another 24 h. Approximately 20 L4 stage $C$. elegans were transferred onto plates at this time. Worms were evaluated for viability every $2-4$ hours for a total of $58 \mathrm{~h}$. As in the previous assays, worms were considered dead when they no longer responded to physical stimuli.

\subsubsection{Statistics and reproducibility}

All experiments were performed in triplicate. Killing curves represent the mean of three separate experiments. Data were analyzed using one-way analysis of variance (ANOVA) with a $P$-value of 0.05 using the statistical software package SPSS (Chicago, IL). Since the starting number of worms was different in each case, the percent alive rather than actual number of worms was used for comparison.

\subsection{Results and discussion}

In this study we assess the potential of extracts from $C$. erectus, $C$. viminalis, and B. buceras to reduce nematode death resulting from $P$. aeruginosa infection. Prior work on these plants revealed an effect on the bacterial QS system (Adonizio et al., 2008b; Adonizio et al., 2006); thus the QS-mutant $\Delta l a s R$ was used as a reference strain. Although there is some precedence for testing plant extracts in a nematode model system 
(Rasmussen et al., 2005), this is the first study to evaluate a statistically significant number of worms in both toxin and infection-based assays.

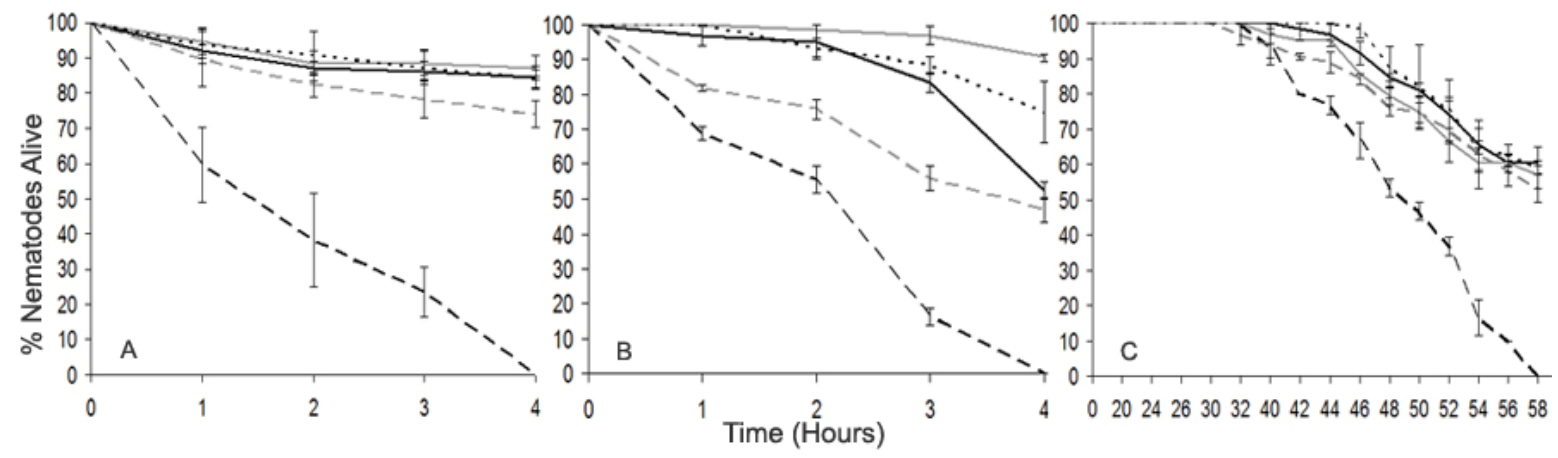

Figure 4.1 Nematode killing curves comparing wt, QS mutant, and wt with plant extract. (A) Paralytic (PAO1), (B) Fast-killing (PA14), and (C) Slow-killing (PA14) assays for $C$. elegans. P. aeruginosa wt (black dash), $\Delta$ las $R$ mutant (grey dash), wt $+C$. viminalis (black dot), wt $+C$. erectus (black line), wt $+B$. buceras (grey line). In all cases, the addition of plant extract rescued the worms to a level at or above a $\Delta$ lasR QS mutant.

\subsubsection{Medicinal plants reduce $C$. elegans paralytic killing by $P$. aeruginosa}

Previous studies have shown that QS signaling is required for maximum levels of worm killing (Mahajan-Miklos et al., 1999; Tan et al., 1999b). Thus, the success of these plant extracts against $P$. aeruginosa strain PAO1 in terms of blocking QS signaling (Adonizio et al., 2008b) suggests they might also reduce death in a PAO1-nematode model. Approximately $50 \%$ of the worms died between hours 1 and 2 after transfer to PAO1 with all nematodes dead after 4 hours (Figure 4.1a). In contrast, all of the worms on E. coli OP50 remained alive throughout the assay. As expected, the QS-mutant 
PAO1 1 las $R$ showed reduced nematode death with $85 \%$ alive between hours 1 and 2 and $74 \%$ still alive at 4 hours (Figure 1a). At the 4-hour mark, approximately $85 \%, 84 \%$, or $87 \%$ of the worms were alive on PAO1 wt plates that contained $C$. erectus, $C$. viminalis, or B. buceras extracts, respectively (Figure 4.1a). In this, and all assays in this study, there was no observable effect of the added plant extracts on worm survival, lifespan, or brood size on E. coli OP50 (not shown), indicating a lack of toxicity of the compounds therein.

All three of the plant extracts, when added to plates containing PAO1 wt, suppressed killing to a level greater than the QS mutant (Figure 4.1a). That is, there was a significant difference between PAO1 $\Delta$ las $R$ and the extract plates $(\mathrm{p}<0.05$ in all cases), but not between individual extracts at the end of the assay. All extract plates and the lasR mutant were significantly different than PAO1 wt without treatment.

Death via PAO1 is due to cyanide asphyxiation and paralysis of $C$. elegans (Gallagher \& Manoil, 2001). The hcn operon in P. aeruginosa mediates cyanide production and is controlled by the QS regulators LasR and RhlR (Pessi \& Haas, 2000). Attenuation of virulence and nematode mortality has been shown with both $\Delta l a s R$ (Darby et al., 1999) and $\Delta h c n$ (Gallagher \& Manoil, 2001) strains. Thus, the results from the paralytic assay suggest that the addition of these extracts are affecting the production of cyanide either through hcn directly; or indirectly via the QS genes. The latter hypothesis agrees with our previous in vitro analysis in PAO1 (Adonizio et al., 2008b) which showed a significant reduction of las $R$ and $r h l R$ gene activity by these extracts. 


\subsubsection{Medicinal plants reduce $C$. elegans fast killing by $P$. aeruginosa}

The results of the fast killing assay are seen in Figure 4.1b. On PA14 wt, approximately $50 \%$ of the worms were dead within two hours, with all worms dead by 4 hours. At this point, all of the worms on OP50 were still alive. Again, the QS-mutant (PA14 LlasR) reduced nematode death, with 75\% alive between hours 1 and 2 and 47\% alive at 4 hours. At the end of the assay, approximately $53 \%, 75 \%$, or $90 \%$ of the worms were alive on PAO1 wt plates with added C. erectus, $C$. viminalis, or B. buceras extracts respectively (Figure 4.1b). As with PAO1, all three of the plant extracts suppressed killing at or above the level of the QS mutant. There was no significant difference between PA14LlasR and the $C$. erectus extract plates at 4 hours $(\mathrm{p}>0.05)$, however $C$. viminalis and B. buceras extracts were significantly better at preventing worm death $(p<$ 0.01 in both cases). Extract-containing plates were all significantly different from each other and but still showed a large reduction in nematode death when compared to PA14 wt without treatment. Overall there is a pronounced inhibitory effect of the plant extracts on $P$. aeruginosa PA14 fast-killing of $C$. elegans.

Fast-killing of $C$. elegans is mediated through production of virulence factors such as phenazines (Mahajan-Miklos et al., 1999). A $\Delta p h n A p h n B$ deletion mutant was shown to completely abolish nematode death, while a TnphoA mutation of the related gene $p h z B$ was shown to greatly reduce mortality in mice and Arabidopsis (MahajanMiklos et al., 1999). Like many virulence factors, phenazines are under control of the QS gene rhlR (Brint \& Ohman, 1995; Latifi et al., 1995). The results from the PA14 fastkilling assay suggest the addition of extracts affect phenazine production, either through the $p h z$ and phn genes or indirectly through the QS system via $r h l$. 
All three extracts were shown previously to significantly affect the $r h l I / R$ system; however, C. erectus has less of an effect on AHL production and biofilm formation (PAO1) (Adonizio et al., 2008b) than either B. buceras or C. viminalis. Though still successful, C. erectus was less efficient in preventing nematode death in the fast-killing assay than the other extracts.

\subsubsection{Medicinal plants reduce $C$. elegans slow killing by $P$. aeruginosa}

The slow killing assay left $50 \%$ of nematodes dead on PA14 wt between hours 48 and 50 with all worms dead at 58 hours. (Figure 4.1c). The control worms on E. coli OP50 were alive throughout the assay. The QS-mutant (PA14 $l a s R)$ reduced nematode death, with $75 \%$ alive between hours 48 and 50 and $53 \%$ alive at 58 hours. At this time, approximately $60 \%, 59 \%$, or $57 \%$ of worms were alive on PAO1 wt plates with added $C$. erectus, C. viminalis, or B. buceras extract respectively (Figure 4.1c). All three of the plant extracts when added to plates containing PA14 wt suppressed killing to the level of the QS mutant. There was no significant difference between PA14 $\Delta l a s R$ and the extract plates or between individual extracts at 58 hours ( $p>0.05$ in all cases): however, all extracts were significantly different from PA14 wt without treatment. This suggests a marked effect of the plant extracts on $P$. aeruginosa infection of $C$. elegans.

Slow-killing of C. elegans occurs over approximately 60 hours due to ingestion of and subsequent infection by $P$. aeruginosa (Tan et al., 1999). Nematode mortality is attenuated with TnphoA-mutations of lasR and gacA (Tan et al., 1999b), suggesting QS is required for the infection process. The addition of plant extracts in this assay drastically reduces nematode death suggesting an effect on lasR or gacA. 
Previous work on these extracts corroborates the inhibitory effect on lasR, however the effect on $g a c A$ was not directly tested (Adonizio et al., 2008b). An effect on either of these factors remains a plausible hypothesis.

\subsection{Conclusions}

All three extracts in all three assays showed a highly significant reduction of virulence when compared to PAO1 and PA14 wt without treatment. Overall, the tested plant extracts reduced nematode death approximately $60-90$ percent by $P$. aeruginosa wt. In each case, this reduction was equal to or greater than that of the corresponding QS mutant strain. The fact that the plant extracts reduce virulence across the board, suggests that they are most likely affecting an upstream QS gene such as las or rhl, or perhaps a global regulator such as gacA. This further corroborates our previous data on the anti-QS effect of these plant extracts (Adonizio et al., 2008b). All extracts inhibit nematode death without significant bactericidal effect, leaving QS inhibition as a plausible hypothesis. In addition, none of the tested plants showed any toxicity in the nematode model, making them reasonable candidates for purification and drug development.

C. erectus, B. buceras, and C. viminalis (and closely related species) have been used medicinally to treat bacterial infection either as teas or poultices (Burkhill, 1985; Irvine, 1961; Melendez, 1982; Morton, 1981; Stewart \& Percival, 1997). Thus, the plants were extracted with hot water to provide greater congruity with traditional preparation methods. Although teas and poultices are many steps removed from modern formulae, traditional use suggests the potential success of topical or enteral routes of administration. 
With the increase in bacterial resistance to antibiotics, we might look to the past in hopes of finding solutions for the future. Plants have been used medicinally for thousands of years, and even without marked antibiotic activity, these three plants are still efficacious in ameliorating disease. We have previously shown the activity of these plants on $P$. aeruginosa alone, and although the exact mechanism of action is not yet known, the nematode experiments described in this paper are consistent with their previous and potential further use as anti-infectives.

\subsection{Acknowledgements}

The authors gratefully acknowledge the support of National Institute of Health, National Center for Alternative and Complementary Medicine NRSA \#1-T32-AT0106001 (A.A., and K.M.) and 1-R15-AT002626-01 (K.M.) NIGMS-RISE program grant R25 GM61347 (A.A.), Cystic Fibrosis Foundation \#ADONIZ06H0 (A.A.), NIAID grants R01 AI072508 and R01 AI064332 (F.M.A.) and members of the Mathee and Ausubel labs for assistance and support; especially Terry Moy, and Rhonda Feinbaum. 


\section{Chapter 5}

"Ellagitannins from Conocarpus erectus exhibit anti-quorum sensing activity against

\section{Pseudomonas aeruginosa"}

Joint Intellectual Property

of

Massachusetts General Hospital

and

Florida International University 


\subsection{Abstract}

Two stereoisomeric ellagitannins, vescalagin and castalagin were isolated from an aqueous extract of Conocarpus erectus L. (Combretaceae). Structures were confirmed via mass spectrometry and NMR spectroscopy. Both ellagitannins were shown to decrease AHL production, quorum sensing (QS) gene expression, and virulence factor production in Pseudomonas aeruginosa. This is the first report of these compounds being isolated from this species, and also the first report of their activity on the QS system of $P$. aeruginosa.

\section{Keyword Index}

Conocarpus erectus; Combretaceae; quorum sensing inhibition; ellagitannins; castalagin; vescalagin

\footnotetext{
Abbreviations: QS, Quorum Sensing; AHL, Acyl-Homoserine Lactone; OdDHL, N-(3oxododecanoyl)-L-homoserine lactone; BHL, N-butanoyl-L-homoserine lactone; TLC, Thin Layer Chromatography; HPLC, High Pressure Liquid Chromatography; NMR, Nuclear Magnetic Resonance
} 


\subsection{Introduction}

Overuse of antibiotics has led to widespread resistance in pathogenic bacteria including the model organism Pseudomonas aeruginosa. This ubiquitous Gram-negative pathogen is a frequent cause of morbidity and mortality in cystic fibrosis patients (Registry, 2005), and nosocomial infections in burn victims, and immunocompromised individuals (Centers for Disease Control and Prevention, 2004). P. aeruginosa and many other organisms are now highly resistant to current antibiotic treatment exposing the need for alternative methods of inhibition.

In our previous work (Adonizio et al., 2008a; Adonizio et al., 2008b; Adonizio et al., 2006), we have shown a number of medicinal plants, including Conocarpus erectus, to be effective in inhibiting pathogenicity of $P$. aeruginosa via attenuation of the quorum sensing (QS) system (for a review of QS see (Fuqua \& Greenberg, 1998)). Addition of C. erectus to culture media affected a significant reduction of QS gene expression and virulence factor production in $P$. aeruginosa (Adonizio et al., 2008b). It also prevented $60-90 \%$ of nematode death from this organism in three different killing assays (Adonizio et al., 2008a).

Commonly known as buttonwood, C. erectus has been used throughout the Caribbean, Puerto Rico, and parts of Africa against catarrh, conjunctivitis, diarrhea, syphilis, and gonorrhea (Burkhill, 1985; Irvine, 1961; Melendez, 1982). The activity of this plant on the bacterial QS system may explain its traditional use for these diseases. This work reveals two hydrolyzable tannins, vescalagin and castalagin, to be responsible for anti-QS activity in C. erectus. 
Tannins are widespread throughout the angiosperms (Okuda et al., 1993), conferring structural benefits to the plant while providing protection through antioxidant and antifeedant activity (Forkner et al., 2004; Hagerman et al., 1998). Often classified as 'waste' in natural products chemistry due to their abundance and lack of protein specificity (Zhu et al., 1997), tannins and other polyphenolics have been previously ignored by the pharmaceutical industry. However, recent studies on the health benefits of these types of compounds are creating a resurgence of interest from the medical field (Ferreira et al., 2005; Haslam, 1996; Okuda, 2005; Quideau et al., 2005).

Castalagin and vescalagin were first characterized by Mayer et al (Mayer et al., 1967; Mayer et al., 1970) and belong to a sub-class of hydrolyzable tannins known as $C$ glycosidic ellagitannins derived from gallic acid metabolism (Quideau \& Feldman, 1996). They are highly water-soluble compounds featuring an open-chain glucose core esterified to numerous oxidatively coupled galloyl moieties (specifically a 4,6hexahydroxybiphenoyl (HHBP) unit and a 2,3,5-nonahydroxyterphenoyl (NHTP) unit) (Khanbabaee \& van Ree, 2001). These complex structural units confer stereochemical rigidity to the molecule, and in fact, $C$-glycosidic ellagitannins would seem to have a higher tendency for selective protein interaction than other classes of polyphenolics (Haslam, 1996; Zhu et al., 1997).

Many polyphenolics possess antimicrobial activity potentially explained by inhibition of microbial enzymes, substrate or iron deprivation, or inhibition of oxidative phosphorylation (Scalbert, 1991). However, the same study shows most bacteria are not susceptible to ellagitannins, i.e. these compounds do not seem to have growth inhibition or cidal effects. Furthermore, it has been shown that ellagic acid (a component of 
ellagitannins) can interfere with bacterial quorum sensing (Huber et al., 2004). This, combined with our previous work on the anti-QS activity of $C$. erectus leads to the hypothesis that ellagitannins may function as inhibitors of the bacterial quorum sensing (QS) system.

\subsection{Materials and methods}

\subsubsection{Isolation and verification of active compounds}

\subsubsection{Plant extraction}

Leaves of Conocarpus erectus (Combretaceae), were collected and processed according to methods described previously (Adonizio et al., 2006). Briefly, pulverized plant material was extracted into boiling water, freeze-dried using a lyophilizer, and stored at $-20^{\circ} \mathrm{C}$ until needed.

\subsubsection{Bioassay-guided fractionation}

Anti-QS activity was confirmed in the crude extract (Adonizio et al., 2006) and followed throughout the separation process using the P. aeruginosa PAO1-derived biomonitor strains pPCS1001 and pPCS1002 (Pesci et al., 1997). These strains harbor lacZ fusions to the QS gene promoter regions enabling blue/white selection for QS activity. Bioassays were carried out as previously described (Adonizio et al., 2006) with some modification. Briefly, LB agar plates were seeded with a lawn of one of the biomonitor strains and allowed to dry for 1 hour. Small wells were then cut and aspirated from the agar, and $10 \mu \mathrm{l}$ aliquots of each fraction were pipetted into each well. Wells were checked at $18 \mathrm{~h}$ and $24 \mathrm{~h}$ for zones of QS inhibition. 


\subsubsection{Preliminary thin layer chromatography}

Approximately $1 \mu \mathrm{l}$ of crude extract was spotted to reverse phase thin layer chromatography (TLC) plates (two spots run in tandem). Adequate separation for visualization of three distinct bands from each spot was achieved with an 80:20 $\mathrm{ACN} / \mathrm{H}_{2} \mathrm{O}$ mobile phase containing $0.1 \%$ formic acid (Figure 5.1a). A $1 \%$ ferric chloride stain was applied to one-half of the plate as a phenolic indicator (Figure 5.1b). The other side was overlaid with agar containing one of the aforementioned PAO1 biomonitor strains to indicate anti-QS activity (Figure 5.1c).

\subsubsection{Fractionation methods}

The fractionation of crude aqueous extract prior to HPLC separation is illustrated in Figure 5.2. Separation was patterned after methods developed for wine polyphenolics (Sun et al., 2006). A C18 PrepSep column (Fisher Scientific 11-131-11 5 g / 20 ml) was conditioned with $200 \mathrm{ml}$ methanol followed by $200 \mathrm{ml}$ water at $\mathrm{pH}$ 7. The flow rate of approximately $2 \mathrm{ml} / \mathrm{min}$ was controlled through positive pressure applied via syringe. The crude aqueous extract of $C$. erectus $(0.25 \mathrm{~g})$ was resuspended in $5 \mathrm{ml}$ water, adjusted to $\mathrm{pH} 7$ with sodium hydroxide, and added to the column. Fraction A was eluted with approximately $150 \mathrm{ml}$ water at $\mathrm{pH}$ 7. The column was then washed with $100 \mathrm{ml}$ water and dried under vacuum for several seconds. Fraction B was then eluted with approximately $400 \mathrm{ml}$ ethyl acetate. The column was washed with ethyl acetate and dried under vacuum before elution of fraction $\mathrm{C}$ with $200 \mathrm{ml}$ of acidified methanol. The presence of phenolics in each fraction was monitored by periodically spotting to TLC 
plates coated with ferric chloride reagent. Each fraction was evaporated to dryness before bioassay at $1 \mathrm{mg} / \mathrm{ml}$ concentration.

\subsubsection{HPLC separation}

Fraction A was separated on an Agilent 1200 series LC system (Agilent Technologies, USA) using an Altima C18 column $(5 \mu, 10 \times 250 \mathrm{~mm} ; 100 \mu \mathrm{L}$ injection volume). A water-acetonitrile mobile phase with $0.1 \%$ formic acid was used with a flowrate of $1.5 \mathrm{ml} / \mathrm{min}$. Conditions were as follows: $0-2 \mathrm{~min}, 0 \%$ acetonitrile; $2-47 \mathrm{~min}$, 0-40\% acetonitrile; $47-48 \mathrm{~min}, 40-100 \%$ acetonitrile; $48-50 \mathrm{~min}, 100 \%$ acetonitrile; 50 $51 \mathrm{~min}, 100-0 \%$ acetonitrile. Fractions were collected manually based on absorbance at $313 \mathrm{~nm}$ and tested for anti-QS activity.

\subsubsection{Mass spectrometric analysis}

Direct injection electrospray ionization mass spectroscopy and MS-MS analysis (Esquire 3000+, Ion Trap Mass Spectrometer Bruker Daltonics, Germany) were used for mass identification of vescalagin and castalagin. The isolated peaks 3 and 4 (Figure 5.4) were injected directly into the ESI source with a syringe pump at a flow rate of 0.2 $\mathrm{ml} / \mathrm{min}$. Nebulizer gas was maintained at $7 \mathrm{psi}$. Capillary temperature was set at $300^{\circ} \mathrm{C}$ with a voltage of $3.5 \mathrm{kV}$. Spectra were obtained in negative ion mode with a scanning range of 100-1000 m/z. MS-MS of compounds were also acquired in negative ion mode. Howevr, the conditions were modified by increasing capillary temperature and voltage to $325{ }^{\circ} \mathrm{C}$ and $4 \mathrm{kV}$ respectively. Trap rolling and smart fragmentation settings were activated, and the instrument was set to scan from 50-1000 m/z. Exact mass 
measurements were made with a Waters Q-Tof2 using reserpine as a lock mass. Samples were introduced via LC flow and reserpine flow from a syringe pump was T-ed in. Spectra were obtained in the positive ion mode with a scanning range from 100-1000 $\mathrm{m} / \mathrm{z}$.

\subsubsection{NMR spectroscopy}

NMR spectra were recorded using a Varian Inova $600 \mathrm{MHz}$ FT-NMR spectrometer with $\mathrm{D}_{2} \mathrm{O}$ acidified with d-TFA or $\mathrm{D}_{2} \mathrm{O}:\left[\mathrm{D}_{6}\right]$-acetone $(8: 2)$ as solvents. Proton spectra were obtained using standard parameters. The structure was elucidated using COSY, HMQC, and HMBC, and through comparison with the standard spectra of vescalagin and castalagin.

\subsubsection{Biological assays}

Assays for AHL production, QS gene activity, and virulence factor production (LasA, LasB, and pyoverdin) were carried out as detailed in our previous work (Adonizio et al., 2008b). Samples were tested at the following concentrations: $1 \mathrm{mg} / \mathrm{ml}$ crude extract of $C$. erectus, $40 \mu \mathrm{g} / \mathrm{ml}$ crude extract, $40 \mu \mathrm{g} / \mathrm{ml}$ vescalagin, or castalagin. These additions were compared to a media-only control for the reduction of QS. Prototypic $P$. aeruginosa strain PAO1 (Holloway \& Morgan, 1986), and its promoter-fusion derivatives $\mathrm{P}_{\text {lasR }}-l a c Z$ (pPCS1001), and $\mathrm{P}_{\mathrm{rhlR}}-l a c Z$ (pPCS1002), (Adonizio et al., 2008b; Pesci et al., 1997) were used throughout this study. In addition, Staphylococcus aureus (ATCC \# 12600) was used in the LasA staphylolytic assay. 


\subsubsection{Statistical analysis}

All experiments were performed in triplicate. Data were analyzed using one-way analysis of variance (ANOVA) with a $P$-value of 0.05 using the statistical software package SPSS (Chicago, IL).

\subsection{Results and discussion}

This report details the isolation and verification of two $C$-glycosidic ellagitannins, castalagin and vescalagin, from $C$. erectus and the confirmation of anti-QS activity of these compounds. The isolation procedure was largely directed by anti-QS bioassays using $P$. aeruginosa strains containing a lacZ fusion to the QS gene lasR or rhlR (Adonizio et al., 2008b; Pesci et al., 1997). Each fraction was pipetted into a small well in an agar plate seeded with one of these biomonitor strains and the appropriate reagents for visualization of lacZ activity. Active fractions resulted in a change of color in the biomonitor strain from blue to off-white in the area surrounding the well indicating antiQS activity. Only fractions with activity were subjected to further separation.

\subsubsection{Isolation and verification of active compounds}

\subsubsection{TLC reveals a phenolic compound responsible for anti-QS activity}

Prior to column chromatography, separation was attempted with various mobile phases on thin layer chromatography (TLC) plates. Reverse phase TLC of crude extract of $C$. erectus using an acidified acetonitrile/water mobile phase revealed two long-wave UV-reactive bands and one chromatic band, the latter being brown in color and slightly tailing (Figure 5.1a). 


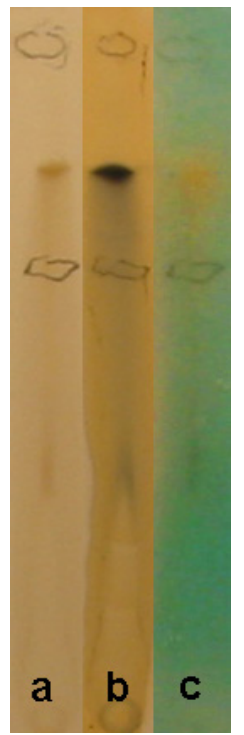

Figure 5.1 Thin layer chromatography (TLC) of C. erectus crude extract and the visualization of phenolic anti-QS activity. (a) Two long-wave UV-reactive bands (circled in pencil) and one chromatic band are visible after running with an acidified 80:20 ACN/ $\mathrm{H}_{2} \mathrm{O}$ mobile phase (RP-C18 silica plates). (b) Phenolic compounds are indicated by a blue spot after reaction with ferric chloride stain. (c) Overlay of the TLC plate with agar containing PAO1 derived biomonitor strain pPCS1001 ( $\left.\mathrm{P}_{\text {lasR }}-l a c Z\right)$ indicates antiQS activity (loss of blue color) correlating with phenolic band.

The plates were reacted with a number of different agents, the most notable being ferric chloride, a stain for phenolic compounds. Ferric chloride staining resulted in the chromatic band turning dark blue, indicating phenolic compounds in this region (Figure 5.1b). An unstained portion of the TLC plate was overlaid with agar containing an antiQS biomonitor strain derived from P. aeruginosa $\left(\mathrm{P}_{\text {lasR }}\right.$-lacZ (pPCS1001)) (Adonizio et al., 2008b; Pesci et al., 1997). Anti-QS activity was visualized as a loss of blue color over 
the phenolic band due to reduced las $R$ expression and linked $\beta$-galactosidase activity (Figure 5.1c).

Anti-QS activity against the tested lasR biomonitor strain correlates with our previous data on the reduction of las $R$ gene expression seen with the crude extract of $C$. erectus (Adonizio et al., 2008b). The localization of this activity indicates phenolic compounds are responsible for the anti-QS activity seen in this species. Phenolic compounds have been previously shown to interfere with bacterial QS (Huber et al., 2004).

\subsubsection{Fractionation of polyphenolics in crude extract}

Since the TLC staining and overlay procedure revealed the phenolic band to contain the anti-QS activity in C. erectus, larger-scale fractionation was then tailored to separation of these compounds. We adopted a method based on the resolution of wine polyphenols (Sun et al., 2006) . A schematic of fractionation can be seen in Figure 5.2.

Crude aqueous extract of $C$. erectus was separated into three fractions based on solvent polarity. Fraction A eluted with water as a bright yellow liquid believed to contain phenolic acids and hydrolyzable tannins according to prior work on polyphenolic separation (Oszmianski et al., 1988; Sun et al., 2006). Fraction B, which eluted with ethyl acetate, was colorless to pale yellow and likely contained colorless proanthocyanins, flavanols, and some monomer and oligomer phenolic acids (He et al., 2006; Oszmianski et al., 1988; Sun et al., 2006). Fraction C, which eluted with acidified methanol, was dark brown indicating the presence of complex tannins, pigmented proanthocyanidins, and pyranoanthocyanins (He et al., 2006; Oszmianski et al., 1988; Sun et al., 2006). The 
presence of phenolics in each fraction was monitored by periodically spotting to TLC plates coated with ferric chloride reagent. Each fraction was tested for anti-QS effect revealing Fraction A to contain the majority of activity (Figure 5.3, (Panel I)).

\section{Crude Extract (pH 7)}

1

Sep-pak C-18 (pH 7)

Water (pH 7)

Peak 1 $\longrightarrow$ Bioassay

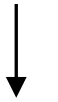

Peak $2 \longrightarrow$ Bioassay

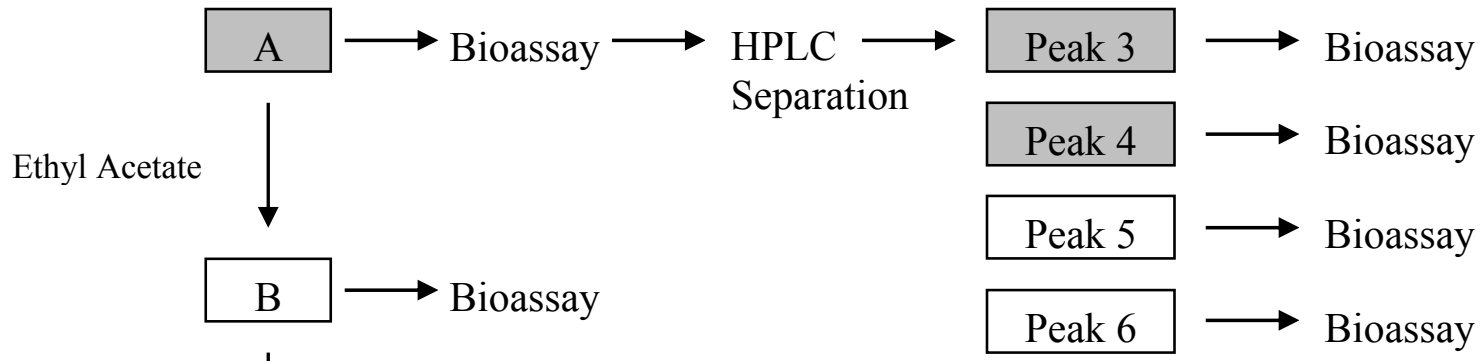

Methanol (pH 2)

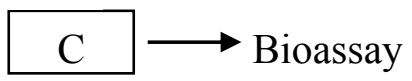

Figure 5.2 Schematic of fractionation of $C$. erectus crude extract. The crude extract and all fractions were tested for anti-QS activity on PAO1-derived biomonitor strains pPCS1001 ( $\Delta l a s R)$ and pPCS1002 ( $\Delta r h l R)$ (Adonizio et al., 2008b; Pesci et al., 1997). Anti-QS activity is indicated by grey blocks. 


\section{A}

B

C

3

Panel I

Panel II

Figure 5.3 Anti-QS bioassay of fractionation products of C. erectus. Anti-QS activity was monitored using PAO1-derived biomonitor strain pPCS1001 (AlasR) (Adonizio et al., 2008b; Pesci et al., 1997). Lighter color indicates anti-QS activity. Fractions A, B, and C were separated by preparatory chromatography (Panel I). Subsequent fractions 1-6 of initial fraction A were separated via HPLC (Panel II).

Fraction $\mathrm{A}$ is the most polar fraction and thus contains phenolic acids and hydrolyzable tannins. Prior work on ellagic acid and EGCG (a hydrolyzable tannin) indicated that these compounds outperformed complex tannins (such as those found in Fraction C) in the inhibition of QS (Huber et al., 2004).

\subsubsection{HPLC separation reveals two fractions with anti-QS activity}

The separation of fraction A via HPLC resulted in six major peaks designated 1 to 6, and a number of minor peaks (Figure 5.4). The detection wavelength was set at 313 $\mathrm{nm}$ based on work by Oszmianski et al (Oszmianski et al., 1988). Fractions 1 through 6 eluted at approximately $24,26.8,29,31.7,33.8$, and 36.4 minutes, respectively. Each 
fraction was collected and tested with the anti-QS biomonitor strains revealing activity in Fractions 3 and 4 (Figure 5.3, (Panel II)).

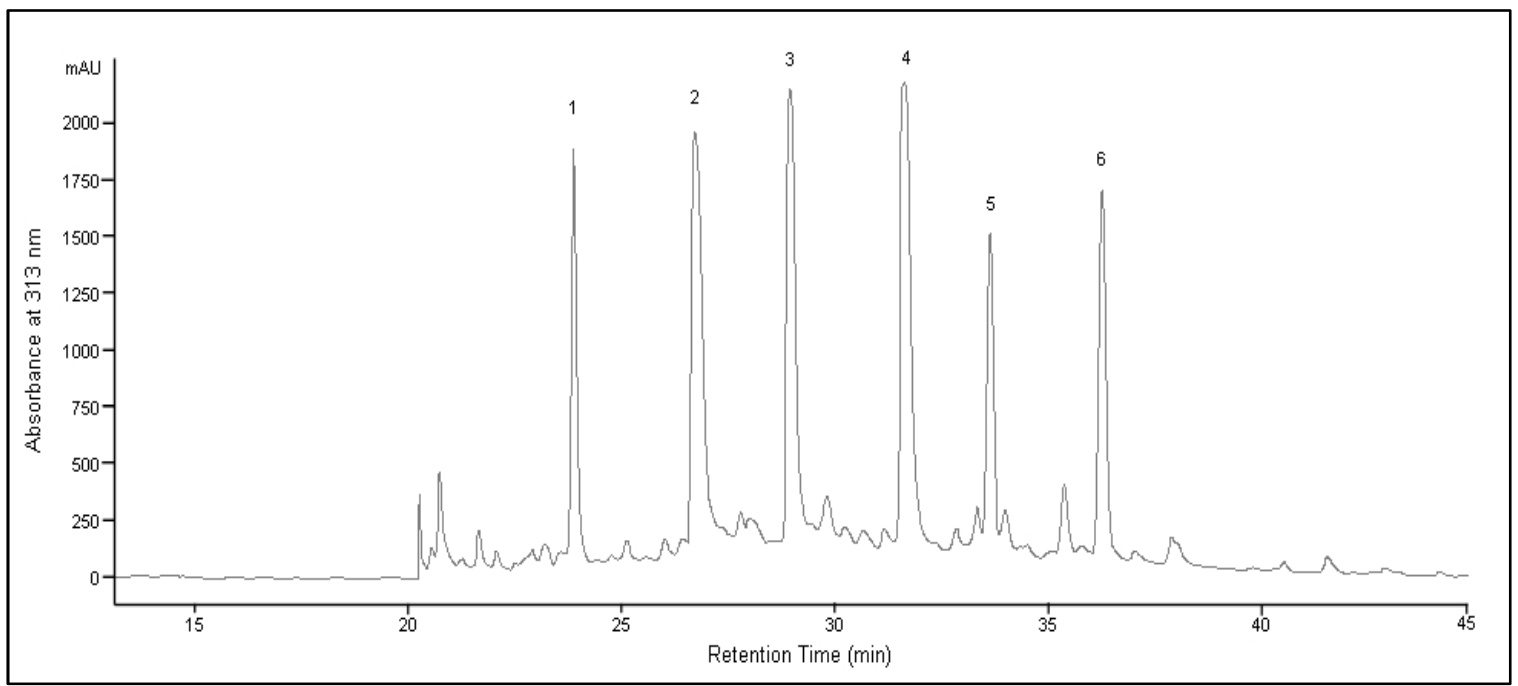

Figure 5.4 HPLC separation of Fraction A. Optimal separation was achieved using an Altima C18 column $(5 \mu, 10 \times 250 \mathrm{~mm} ; 100 \mu \mathrm{L}$ injection volume $)$ with an acidified wateracetonitrile mobile phase. Absorbance was monitored at a wavelength of $313 \mathrm{~nm}$. Fractions 1-6 represent pure compounds and were subsequently tested for anti-QS activity.

\subsubsection{Vescalagin and castalagin elucidated as active compounds}

Fractions 3 and 4 were checked for purity by TLC and behaved as pure compounds (single, non-tailing bands). Both Fractions 3 and 4 when subjected to mass spectrometric analysis produced a strong peak at m/z $933[\mathrm{M}-\mathrm{H}]^{-}$and a smaller fragment peak at $\mathrm{m} / 2 \mathrm{z} 466[\mathrm{M}-\mathrm{H}]^{2-}$. 
MS-MS of compounds 3 and 4 revealed strong peaks at m/z 915 and 613 . The former being simply the parent compound minus water, and the latter indicating the loss of ellagic acid (302 daltons), a fragment regarded as diagnostic of ellagitannins (Tang \& Hancock, 1995). A literature review suggested that these compounds may be the ellagitannins vescalagin and castalagin (Mayer et al., 1967; Mayer et al., 1970; Okuda et al., 1993; Tang \& Hancock, 1995) as they both have a mw of 934 and contain ellagic acid components. Exact mass measurements revealed a mass of $935.0811[\mathrm{M}+1]$ for Fraction 3 and 935.0794 for Fraction $4[\mathrm{M}+1]$, a difference of 2.8 and $1.0 \mathrm{ppm}$, respectively. The calculated exact mass for the $[\mathrm{M}+1]$ ion of both vescalagin and castalagin is: 935.0785 for $\mathrm{C}_{41} \mathrm{H}_{27} \mathrm{O}_{26}{ }^{+}$.
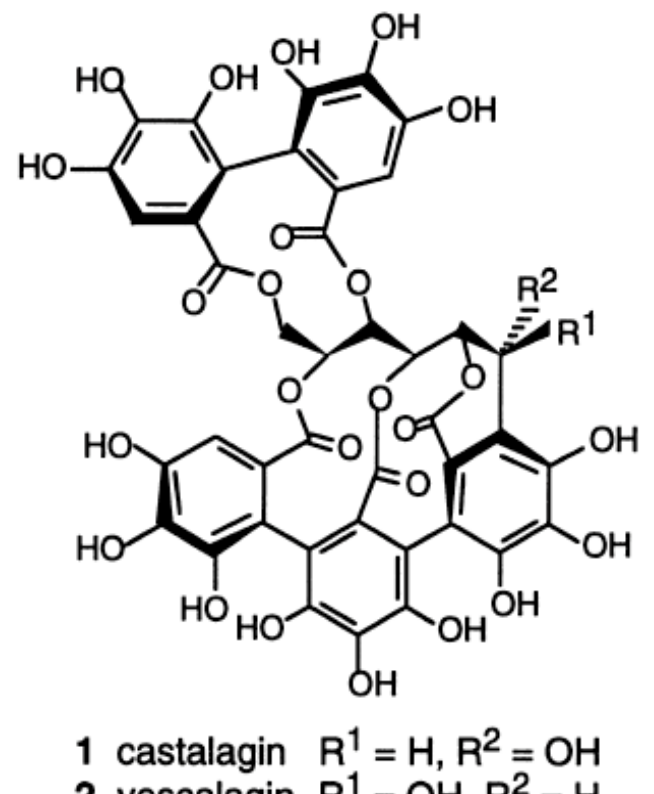

2 vescalagin $R^{1}=O H, R^{2}=H$

Figure 5.5 Structures of the stereoisomeric ellagitannins castalagin and vescalagin. (Image: (Zhentian et al., 1999)) 
NMR data (supplementary data, appendix) were compared with spectra from the known compounds (Glabasnia \& Hofmann, 2006; Tang \& Hancock, 1995), thus confirming the identity of Fractions 3 and 4 as vescalagin and castalagin, respectively (Figure 5.5). To avoid confusion, these fractions will hereafter be referred to by their compound names.

\subsubsection{Bioassays on P. aeruginosa confirm anti-QS activity of ellagintannins}

The anti-QS activity of the ellagitannins vescalagin and castalagin is corroborated by prior work on the QS-inhibiting properties of ellagic acid (Huber et al., 2004). However, specific bioassays were necessary to elaborate the precise effect on $P$. aeruginosa QS and virulence. Here, we compare the effect of the purified compounds to that of the crude extract of $C$. erectus examined in our previous works (Adonizio et al., 2008a).

Polyphenolics have been shown to act as QS-inhibitors at a concentration range of $20-60 \mu \mathrm{g} / \mathrm{ml}$ (Huber et al., 2004) thus a $40 \mu \mathrm{g} / \mathrm{ml}$ concentration was chosen for the purified ellagitannins. For comparison, we tested $40 \mathrm{mg} / \mathrm{ml}$ and $1 \mu \mathrm{g} / \mathrm{ml}$ concentrations of crude extract, the latter being the working concentration for our previous studies on of C. erectus (Adonizio et al., 2008a; Adonizio et al., 2008b).

\subsubsection{LasA protease activity is reduced in the presence of ellagitannins}

LasA belongs to the $\beta$-lytic endopeptidase class of proteases (Kessler, 1995) and plays a major role in host tissue degradation (Kharazmi, 1989; Morihara \& Homma, 1985). LasA protease activity was determined by measuring the ability of culture 
supernatants to lyse boiled $S$. aureus cells (Kong et al., 2005). There was a significant decrease in LasA activity compared to that of the control when strain PAO1 was grown in the presence of C. erectus crude extract at $1 \mathrm{mg} / \mathrm{ml}$ (91\% decrease), and a lesser effect at $40 \mu \mathrm{g} / \mathrm{ml}$ (26\% decrease) (Table 5.1). Purified vescalagin and castalagin affected significant reductions in LasA activity as well, with decreases of $73 \%$ and $80 \%$, respectively (Table 5.1). The significant effect on LasA protease production is in agreement with our previous data on C. erectus (Adonizio et al., 2008b), and suggests that vescalagin and castalagin are responsible for the reduction in LasA activity.

\subsubsection{LasB elastase activity is reduced in the presence of ellagitannins}

LasB elastase is a zinc metalloprotease capable of affecting the host immune system and destroying biological tissue (Bever \& Iglewski, 1988). The elastolytic activity of culture supernatants was determined using elastin Congo red (ECR; Sigma, St. Louis, MO) (Ohman et al., 1980). There was a significant decrease in LasB activity compared to that of the control when PAO1 was grown in the presence of C. erectus at $1 \mathrm{mg} / \mathrm{ml}(70 \%$ decrease) or $40 \mu \mathrm{g} / \mathrm{ml}$ (60\% decrease) (Table 5.1). Purified vescalagin and castalagin also affected significant reductions in LasB activity, with decreases of $67 \%$ and $63 \%$, respectively (Table 5.1). The effect on LasB production agrees with our previous data (Adonizio et al., 2008b) on C. erectus, and suggests vescalagin and castalagin are responsible for the reduction in LasB activity.

Previous studies by Huber et. al. indicate a $40 \%$ decrease in lasB activity when the related organism Pseudomonas putida is grown in the presence of $30 \mu \mathrm{g} / \mathrm{ml}$ ellagic acid (a component of ellagitannins) (Huber et al., 2004). 
Table 5.1 Effect of crude extract of $C$. erectus and purified ellagitannins on $P$. aeruginosa virulence factors

\begin{tabular}{lccc}
\hline Culture conditions & LasA activity $^{\mathrm{a}}$ & Elastase activity $^{\mathrm{b}}$ & Pyoverdin production $^{\mathrm{c}}$ \\
\hline Media only & $0.243 \pm 0.024$ & $142.7 \pm 17.7$ & $4710 \pm 238$ \\
Crude $1 \mathrm{mg} / \mathrm{ml}$ & $0.021 \pm 0.004^{*}$ & $42.9 \pm 2.1^{*}$ & $700 \pm 212^{*}$ \\
Crude $40 \mu \mathrm{g} / \mathrm{ml}$ & $0.179 \pm 0.013^{*}$ & $56.7 \pm 5.5^{*}$ & $4208 \pm 205$ \\
Vescalagin & $0.066 \pm 0.008^{*}$ & $47.2 \pm 6.8^{*}$ & $4226 \pm 138$ \\
Castalagin & $0.048 \pm 0.003^{*}$ & $52.7 \pm 9.0^{*}$ & $4207 \pm 150$ \\
\hline
\end{tabular}

${ }^{\mathrm{a}}$ Las $\mathrm{A}$ activity was expressed as reduction in $\mathrm{OD}_{600}$ per hour per microgram total protein

${ }^{\mathrm{b}}$ Elastase activity was expressed as absorbance at $\mathrm{OD}_{495}$ per microgram of protein $* 1000$

${ }^{\mathrm{c}}$ Pyoverdin production expressed as fluorescence $(405 / 465 \mathrm{~nm})$ per microgram protein *Significantly different from the "Media only" control at $p=0.05$

\subsubsection{Ellagitannins do not affect pyoverdin production}

Pyoverdins function as siderophores essentially starving host tissues by competing with mammalian transferrin for iron (Meyer et al., 1996). They also promote pathogenicity by stimulating bacterial growth (Cox \& Adams, 1985), while autoregulating themselves and the production of other toxins (Beare et al., 2003; Lamont et al., 2002). Relative concentration of pyoverdin was based on fluorescence of the supernatant at an excitation wavelength of $405 \mathrm{~nm}$ and an emission wavelength of 465 nm (Gemini EM microplate reader). None of the experimental conditions significantly affected pyoverdin production, with the exception of $C$. erectus at a concentration of 1 
$\mathrm{mg} / \mathrm{ml}$ (85\% reduction) (Table 5.1). This is consistent with our previous data on $C$. erectus (Adonizio et al., 2008b).

The inability of the purified compounds to reduce pyoverdin levels suggests that the crude extract contains some factor other than the ellagitannins that is responsible for this effect. No significant reduction occurred with a crude extract concentration of 40 $\mu \mathrm{g} / \mathrm{ml}$, indicating a relatively high amount of this unknown factor is needed for an effect on pyoverdin levels.

\subsubsection{Ellagitannins affect the production of QS signaling molecules}

$P$. aeruginosa manufactures two main quorum sensing signaling molecules $N$-(3oxododecanoyl)-L-homoserine lactone (OdDHL) and $N$-butanoyl-L-homoserine lactone (BHL) called autoinducers (Pearson et al., 1994; Pearson et al., 1995). These molecules

diffuse into the environment, and when they reach a putative threshold concentration, they activate the QS receptor genes. Inhibition of these signals has been shown to cause attenuation of pathogenicity (Adonizio et al., 2008a; Adonizio et al., 2008b; Manefield et al., 1999; Whitehead et al., 2001b).

No significant decreases in OdDHL levels were seen with either the crude extract or the purified compounds (Table 5.2), the former being consistent with our previously acquired data (Adonizio et al., 2008b). BHL levels however, were affected by the crude extract at a concentration of $1 \mathrm{mg} / \mathrm{ml}$ (20\% reduction) and purified vescalagin $(21 \%$ reduction). This is consistent with our previous work on $C$. erectus which revealed a $25 \%$ decrease in BHL levels, suggesting that vescalagin is responsible for this reduction. 
Table 5.2 Effect of ellagitannins on P. aeruginosa QS genes and AHL production

\begin{tabular}{|c|c|c|c|c|c|c|}
\hline \multirow{2}{*}{ Culture conditions } & \multicolumn{2}{|c|}{ AHL Production ${ }^{\mathrm{a}}$} & \multicolumn{4}{|c|}{ Gene Expression $^{\mathrm{b}}$} \\
\hline & C12-AHL & C4-AHL & lasI & las $R$ & rhlI & $r h l R$ \\
\hline Media only & $1.328 \pm 0.14$ & $0.621 \pm 0.03$ & $3873 \pm 260$ & $4823 \pm 385$ & $4933 \pm 333$ & $7639 \pm 216$ \\
\hline Crude $1 \mathrm{mg} / \mathrm{ml}$ & $1.082 \pm 0.07$ & $0.496 \pm 0.05^{*}$ & $3065 \pm 247 *$ & $1325 \pm 275^{*}$ & $3383 \pm 111^{*}$ & $4535 \pm 231 *$ \\
\hline Crude $40 \mu \mathrm{g} / \mathrm{ml}$ & $1.431 \pm 0.06$ & $0.578 \pm 0.08$ & $3367 \pm 276$ & $2873 \pm 188^{*}$ & $3187 \pm 249 *$ & $4667 \pm 194^{*}$ \\
\hline Vescalagin & $1.227 \pm 0.16$ & $0.489 \pm 0.01 *$ & $2049 \pm 93^{*}$ & $1703 \pm 297^{*}$ & $3514 \pm 306^{*}$ & $4129 \pm 287^{*}$ \\
\hline Castalagin & $1.389 \pm 0.20$ & $0.703 \pm 0.01$ & $2465 \pm 116^{*}$ & $2160 \pm 509 *$ & $4542 \pm 199$ & $4904 \pm 348^{*}$ \\
\hline \multicolumn{7}{|c|}{${ }^{\mathrm{a}} \mathrm{AHL}$ production was expressed in $\mu \mathrm{M}$} \\
\hline \multicolumn{7}{|c|}{${ }^{b}$ Gene expression was measured via $\beta$-galactosidase activity of the lac $Z$ gene fusion } \\
\hline
\end{tabular}

\subsubsection{Ellagitannins affect QS gene expression}

P. aeruginosa elaborates two main sets of QS systems: lasI-lasR and rhlI-rhlR (Schuster \& Greenberg, 2006). LasI and RhlI are synthetases that manufacture the autoinducer signaling molecules mentioned in the previous section. The receptors, las $R$ and $r h l R$, are activated by these signals and, in turn, coordinate the regulation of pathogenicity.

We tested the effect of castalagin and vescalagin on the transcriptional activity of the QS-gene promoters using PAO1-derived strains harboring promoter-lacZ fusions (as described in (Kong et al., 2005) and (Adonizio et al., 2008b). Assays for $\beta$-galactosidase 
(lacZ) activity in $P$. aeruginosa were performed with $o$-nitrophenyl- $\beta$-Dgalactopyranoside, as described previously (Mathee et al., 1997).

Significant effects were seen on the activity of all tested QS genes with C. erectus at $1 \mathrm{mg} / \mathrm{ml}$ (Table 2) which agrees with previous results on this species (Adonizio et al., 2008b). Crude extract at $40 \mu \mathrm{g} / \mathrm{ml}$ reduced the expression of all QS genes to a lesser extent, leaving lasI marginally insignificant. Castalagin reduced all QS gene levels save for rhlI which may correspond to the lack of effect on its signaling molecule OdDHL (Table 1). Vescalagin on the other hand reduced all QS gene levels including rhlI to a greater extent than castalagin. The significant reduction of rhlI by vescalagin may correspond to its effect on BHL.

Interestingly, reduction of lasI is more pronounced with the addition of pure compounds than with the crude extract (Table 2). This may suggest another compound in the crude extract causing up-regulation of lasI or simply blocking its repression by the ellagitannins thus balancing out the effect.

\subsubsection{Ellagitannins do not affect bacterial growth}

To confirm that the reduction in virulence was due to QS inhibition and not static or cidal effects, cell proliferation was monitored using growth curve studies and the Bradford assay (Bradford, 1976). No significant differences in growth of PAO1 were seen with either concentration of crude extract or the purified compounds. In comparison, previous work on ellagic acid shows no effect on growth of the related species $P$. putida at concentrations up to $30 \mu \mathrm{g} / \mathrm{ml}$ (Huber et al., 2004). 


\subsection{Concluding remarks}

Previous research on $C$. erectus (Adonizio et al., 2008a; Adonizio et al., 2008b) indicates a marked reduction of QS and inhibition of $P$. aeruginosa virulence. The activity of the isolated compounds vescalagin and castalagin are shown here to account for the majority of the activity in this plant, suggesting a new mode of action for ellagitannins. Purified ellagitannins affected AHL levels, and QS-gene expression similarly to the parent extract. Protease and elastase levels were also markedly reduced, however, pyoverdin was not affected. This may suggest that additional compounds within C. erectus are responsible for the entire anti-QS effect or that the regulation of pyoverdin extends beyond the QS system. The latter hypothesis is supported by previous research on the nature of pyoverdin (Beare et al., 2003; Lamont et al., 2002) and the mixed results of halogenated furanones on its production (Hentzer et al., 2003; Sakar et al., 2005).

Unlike the furanones, which inhibit QS through competitive AHL inhibition, ellagitannins are unlikely candidates for signal mimicry based on their size and structure. The reduction of rhlI expression and BHL levels by vescalagin (Table 2) may suggest a more direct effect on the $r h l$ QS system, however further studies are necessary to confirm this activity.

In several cases (Table 1 and 2), differences in the degree of anti-QS activity between the crude extract and the purified compounds can be explained by a dosage effect. The extraction of vescalagin and castalagin from the crude extract was found to be roughly $6 \%$ in both cases with our particular methods. Thus, the working concentration of ellagitannins is as follows: $60 \mu \mathrm{g} / \mathrm{ml}$ in the $1 \mathrm{mg} / \mathrm{ml}$ crude extract and $2.4 \mu \mathrm{g} / \mathrm{ml}$ in the $40 \mu \mathrm{g} / \mathrm{ml}$ crude extract. The tested concentration of the purified compounds falls 
between these two as does the activity in most cases. This pattern is not absolute however, since unknown compounds in the crude extract may moderate activity.

Castalagin and vescalagin have not been previously isolated from Conocarpus erectus, and although they may be part of a larger $C$-glycosidic ellagitannin oligomer, it is likely these compounds exist in their native state as well. Although tannins are widespread throughout the plant kingdom, NHTP-bearing ellagitannins such as castalagin and vescalagin are mostly limited to the Combretaceae, Fagaceae, Melastomataceae, and Myrtaceae (Okuda et al., 2000). Interestingly, the plant species found in our previous work to have the highest levels of anti-QS activity are within these four families Conocarpus erectus L. (Combretaceae), Callistemon viminalis (Sol.ex Gaertn.) G. Don (Myrtaceae), Bucida burceras L. (Combretaceae), Tetrazygia bicolor (Mill.) Cogn. (Melastomataceae), and Quercus virginiana Mill. (Fagaceae). (Adonizio et al., 2008b). This would suggest that similar ellagitannins may be responsible for QS inhibition in these plants.

\subsection{Acknowledgments}

We gratefully acknowledge the support of the National Institute of General Medical Sciences - Research Initiative for Scientific Enhancement (NIGMS-RISE) program \#R25 GM61347 (A.A.), the Cystic Fibrosis Foundation Traineeship Grant \#ADONIZ06H0 (A.A.), and the National Institute of Health, National Center for Alternative and Complementary Medicine NRSA \#1-T32-AT01060-01 (A.A., and K.M.) and 1-R15-AT002626-01 (K.M.), and NIAID grants R01 AI072508 and R01 AI064332 (F.M.A.) Special thanks go out to Monica Pupo and members of the Clardy lab for NMR 
interpretation, and Hanna Milewicz, Tomo Kawate, and Steven Casper for chromatography advice. We would also like to thank the reviewers for their critical reading of this manuscript and their invaluable suggestions and comments 
Chapter 6

General Discussion and Summary 


\subsection{Overview}

With the advent of antibacterial therapy in the 1940s came the consequent problem of bacterial resistance (Abraham et al., 1941). Early $\beta$-lactam antibiotics such as penicillin quickly lost efficacy, and by the 1960s new drugs were being developed to replace the old (Fairbrother \& Taylor, 1961). Soon, they too would become obsolete, and so began the Sisyphean task of keeping pathogens at bay.

The search for novel antibiotics is becoming increasingly necessary, though perhaps more important is the search for novel modes of action against pathogens. A compound that does not kill an organism, but rather attenuates its virulence could allow the host immune system to more efficiently clear out an infection. In addition, this type of action against bacteria is less likely to provide the selective pressure to drive resistance (Hentzer \& Givskov, 2003b). Anti-QS agents seem to fit this niche, but are limited in number and structural diversity.

This dissertation has served not only to direct research toward ethnobotanical sources of anti-QS activity (Chapter 2; (Adonizio et al., 2006)), but has confirmed the hypothesis that anti-QS compounds from medicinal plants can attenuate $P$. aeruginosa virulence. A number of plants were shown to reduce the production of virulence factors, biofilm formation, and QS activity in this intractable, opportunistic pathogen (Chapter 3; (Adonizio et al., 2008b)). Furthermore, three of these plants were able to prevent $P$. aeruginosa infection and toxicity in a live animal model (Chapter 4; (Adonizio et al., 2008a)). The ellagitannins vescalagin and castalagin were found to be responsible for the majority of anti-QS activity in the medicinal plant C. erectus (Chapter 5) revealing a class of compounds not previously tagged with this effect. 


\subsection{Anti-QS activity of medicinal plants}

The plant kingdom has long been a source of medicines, and the relatively untapped botanical arena of southern Florida seemed a likely reservoir for anti-QS compounds. The ability of higher organisms to produce QS inhibitors was first demonstrated in the marine algae Delisea pulchra (Manefield et al., 1999), and anti-QS activity has since been found in a number of higher plants (Teplitski et al., 2000). However, the screening of medicinal plants for this activity has not been explored prior to this study, and this research sought to forge a new path in the field of medical ethnobotany.

Fifty medicinal plants of southern Florida were screened for their anti-QS potential against Chromobacterium violaceum and Agrobacterium tumefaciens (NTL4) biomonitor strains in a standard disc-diffusion assay (Chapter 2; (Adonizio et al., 2006)). These biomonitor strains provided a colorimetric assessment of QS inhibition seen as a zone of pigment loss surrounding the test disc (Chapter 2, Figure 2.2).

Although the biomonitor strains rarely cause disease in humans, they remain medically relevant due to the similarity of Gram negative QS systems (Whitehead et al., 2001a). It can be extrapolated that a plant effective against the QS systems of these species may have activity against disease-causing organisms as well thus validating the medicinal use of these plants.

Of the tested plants, six showed QS inhibition: Conocarpus erectus (Combretaceae), Chamaecyce hypericifolia (Euphorbiaceae), Callistemon viminalis (Myrtaceae), Bucida burceras (Combretaceae), Tetrazygia bicolor (Melastomataceae), and Quercus virginiana (Fagaceae). 
C. erectus and B. buceras both belong to the family Combretaceae. Leaves from these tree species have been used medicinally to treat fevers, respiratory ailments, diarrhea and sexually transmitted diseases throughout the native range of these plants (Burkhill, 1985; Irvine, 1961; Melendez, 1982; Morton, 1981; Nellis, 1994).

C. viminalis, of the family Myrtaceae, is now considered to be in the genus Melaleuca. This group includes the "tea trees" (Chapter 1.2.1), which have long been used to treat skin and respiratory conditions (Duke, 1985; Halcon \& Milkus, 2004; Hammer et al., 1996; Melendez, 1982; Morton, 1981). Results in this study suggest the antimicrobial action of $C$. viminalis and related species might be due, in part, to anti-QS activity.

Q. virginiana and T. bicolor are trees of the Fagaceae and Melastomataceae respectively. Q. virginiana has a long history of use by Cuban peoples and Native American tribes as a febrifuge and for dysentery (Moerman, 1998; Morton, 1981), whereas T. bicolor has been used to treat fevers in the Bahamas (Morton, 1981). As suggested by this work, traditional use may be related to QS inhibition.

C. hypericifolia (Euphorbiaceae), is used in the treatment of opthamalogical ailments, diarrhea and other digestive disorders, respiratory conditions, fever, and gonorrhea. This latex-containing, weedy species is found throughout the Caribbean, South American, and old world tropics. Interestingly, only the ethanol extract of this plant exhibited anti-QS activity, suggesting the active compounds to be either less polar than those found in the other tested plants, or possibly heat labile. 


\subsection{Attenuation of Pseudomonas aeruginosa virulence}

After displaying promising anti-QS activity against several biomonitor strains (Chapter 1), six plants from that study were tested against the human opportunistic pathogen $P$. aeruginosa.. Relevant to the traditional uses of these plants, $P$. aeruginosa may indeed be the causative agent for a number of conditions such as fever, eye and skin infections, and respiratory ailments (Bodey et al., 1983) (Chapter 1, Figure 1.10). Additionally, this organism remains problematic, especially in the immunocompromised individual, due to its intrinsic and inherent resistance, massive exotoxin production, and the ability to form biofilms (Hancock \& Speert, 2000; Lyczak et al., 2002). The production of toxins and biofilms is QS controlled (Venturi, 2006), and thus attenuation of $P$. aeruginosa virulence should be seen with plant inhibitors of the QS system.

The active plants from the previous study were thoroughly evaluated for their effect on a number of virulence factors, QS-gene expression, and QS signal concentration (Chapter 3; (Adonizio et al., 2008b)). Effect on LasA production was gauged via staphylolytic assay, while LasB inhibition was visualized with chromophore-linked elastin. Pyoverdin concentrations in the supernatant were recorded based on florescence, and biofilm formation was assessed in PVC microtiter plates. Additionally, the direct effect on the main QS system was followed using ONPG visualization of lacZ-QS gene fusion products. AHL signal concentration was also monitored by LC-MS.

The most effective plants, C. erectus, B. buceras, and $C$. viminalis, caused significant inhibition of LasA protease, LasB elastase, pyoverdin production, and biofilm formation (Chapter 3, Table 3.1). Additionally, each plant demonstrated a distinct profile 
of effect on the las and $r h l$ QS genes and their respective signaling molecules (Chapter 3, Table 3.2), suggesting different mechanisms responsible for efficacy. Furthermore, extracts of all plants caused inhibition of QS genes and QS-controlled factors without a significant decrease in cell density. This suggests an inhibition of quorum sensing rather than a bactericidal effect.

At this point, there is not sufficient data to pinpoint the exact method of QS inhibition, however a partial mechanistic picture can be drafted for the top three extracts based on the results in Chapter 3. C. erectus and B. buceras exhibit a similar pattern of QS system repression. Both show a significant decrease in expression of all four QS genes tested (Table 3.2). Both extracts also decrease levels of the signaling molecules OdDHL and BHL, though only B. buceras does so significantly. With a few exceptions, both extracts also markedly reduce virulence and biofilm formation (Table 3.1). This general effect may suggest that the components from these plant extracts could affect a global QS regulator such as GacA (Figure 6.1).

Additional information on the ellagitannins purified from C. erectus (Chapter 5) suggests that vescalagin may have a more direct effect on the rhl system as it significantly reduces both rhlI expression and BHL concentration (Table 5.2). Since neither of the purified ellagitannins had an effect on pyoverdin, there may be a yet unidentified compound in $C$. erectus responsible for its attenuation. However, the unresolved effect of the purified ellagitannins on $r h l R$ may suggest regulation of pyoverdin by factors outside of the QS system.

C. viminalis also generates an interesting profile of effect on $P$. aeruginosa. It shows a pronounced reduction of OdDHL, however no reduction of BHL (Chapter 3, 
Table 3.2). This could suggest a more direct inhibition of LasI, lifting the negative regulation of OdDHL on RhlR thus activating a feedback loop to increase production of BHL (Figure 6.1). The most significant decreases in lasI and lasR expressions were found with $C$. viminalis (Table 3.2). As expected, the LasA and elastase activities are also significantly decreased (Table 3.1).

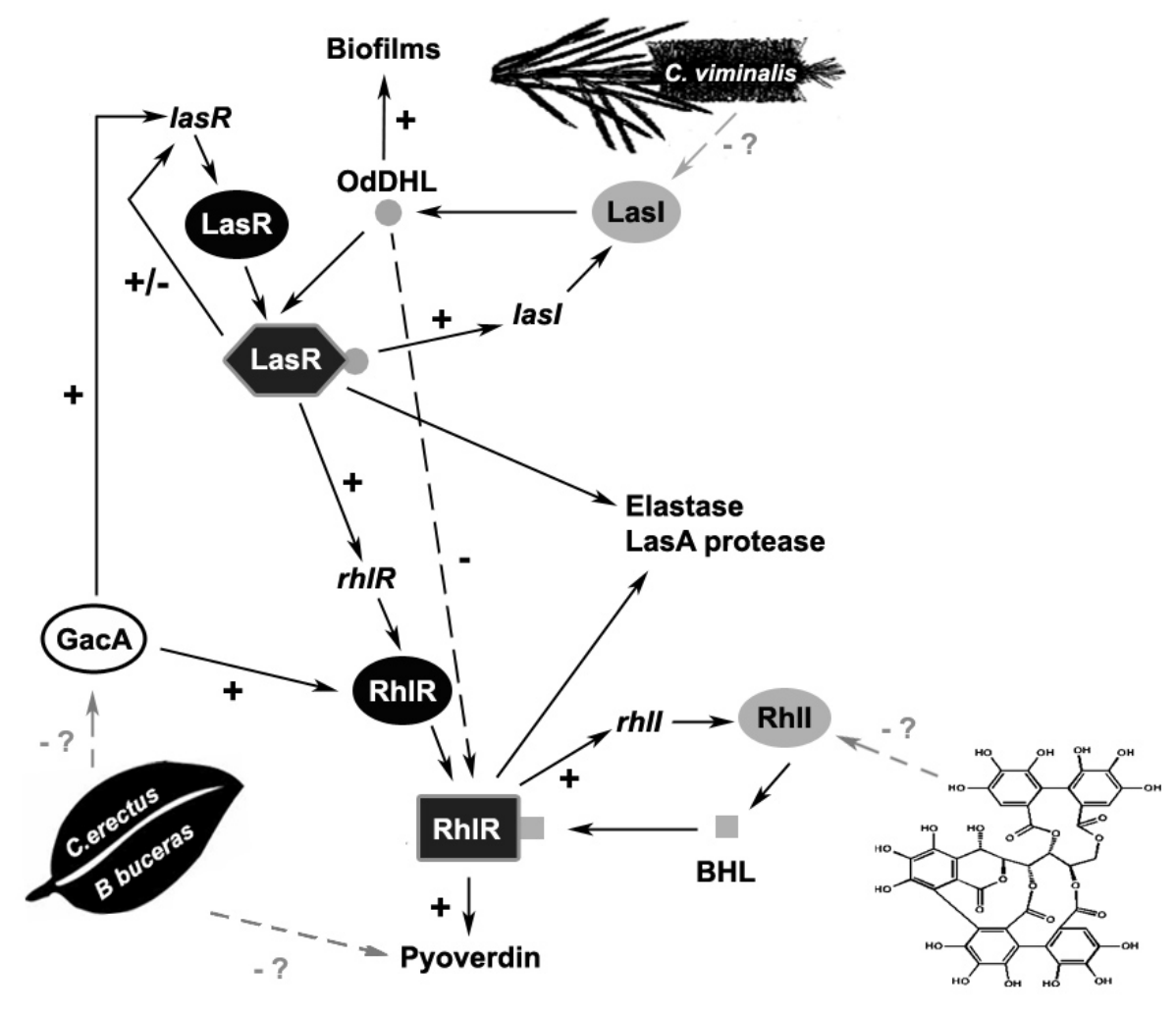

Figure 6.1 QS pathway of P. aeruginosa (modified from Van Delden and Iglweski 1999) and possible activity of crude extracts and purified vescalagin. GacA is a global regulator of QS, LasI is a synthetase which produces the signal OdDHL, LasR is the regulator which binds OdDHL. RhlI synthetase produces BHL which binds to its cognate receptor 
RhlR. Black arrows indicate known regulatory patheays. Grey arrows indicate possible mechanisms of action of $C$. erectus, B. buceras, $C$. viminalis, and vescalagin.

\subsection{Inhibition of $P$. aeruginosa virulence in a nematode model}

Results from in vitro studies, although significant, are often too far removed from a live host to suggest any clinical relevance. Thus, a Caenorhabditis elegans system was chosen to model the effect of the plant extracts on a P. aeruginosa infection (Darby et al., 1999; Tan \& Ausubel, 2000). Identical virulence factors are responsible for pathogenicity in both a human and nematode host (Rahme et al., 1995; Tan et al., 1999a), and in both cases virulence is QS-mediated. Thus, addition of plant compounds that have an effect on P. aeruginosa $\mathrm{QS}$, should therefore attenuate bacterial virulence and subsequent death of C. elegans (Chapter 4; (Adonizio et al., 2008a)).

Aqueous extracts of $C$. erectus, C. viminalis, and B. buceras were evaluated in both toxin-based and infection-based assays with P. aeruginosa strains PAO1 and PA14. Toxin based assays such as paralysis or "Fast-killing" of follow P. aeruginosa killing of C. elegans due to the absorption of virulence factors or cyanide toxicity. "Slow-killing" assays, report death via ingestion of the bacteria and subsequent infection (MahajanMiklos et al., 1999; Tan et al., 1999a; Tan et al., 1999b).

The tested plant extracts prevented mortality via gut infection in almost $60 \%$ of the worms, and caused a 50-90\% reduction in death from toxin production (Figure 4.1). Although there was an unexplored possibility that the plants augmented the immune system of the nematode thus preventing massive infection, it is much more likely to be an anti-QS effect given the previous in vitro results (Chapter 3; (Adonizio et al., 2008b)). 
Additionally, all extracts inhibited nematode death by $P$. aeruginosa without host toxicity, indicating their potential for further development as anti-infectives.

\subsection{Ellagitannins are responsible for anti-QS activity}

After establishing anti-QS activity of the plant extracts on P. aeruginosa both in vitro and in a live animal model, one extract (C. erectus) was chosen for isolation of active compounds. The isolation procedure was largely guided by anti-QS bioassays using $P$. aeruginosa lacZ-fusion strains discussed in Chapter 3 .

Two stereoisomeric ellagitannins, vescalagin and castalagin were isolated from the aqueous extract of $C$. erectus using low-pressure column chromatography and HPLC. Structures were confirmed via mass spectrometry, proton-, and 2D-NMR spectroscopy.

Both ellagitannins were shown to decrease AHL production, QS gene expression, and virulence factor production in P. aeruginosa (Chapter 5, Table $5.1 \& 5.2$ ). This is the not only the first report of these compounds being isolated from C. erectus, but also the first documentation of their activity on the QS system of $P$. aeruginosa.

An interesting retrospective is that all active plants from the first study belong to plant families known to contain ellagitannins (Chapter 5). This would suggest at least some of the active compounds to be ellagitannins in these species. The Combretaceae, Fagaceae, Melastomataceae, Euphorbiaceae, and Myrtaceae families all produce $C$ glycosidic tannins based on ellagic acid. In addition, ellagic acid itself has been shown to possess anti-QS activity (Huber et al., 2004), further corroborating the findings in this study 


\subsection{Future directions}

This work demonstrated a number of medicinally-used plants to have anti-QS activity, most notably Conocarpus erectus (Combretaceae), Bucida buceras (Combretaceae), and Callistemon viminalis (Myrtaceae). These plants effectively inhibited QS-gene expression, signal concentration, and virulence factors in $P$. aeruginosa. Additionally, the attenuation of morbidity and mortality in a nematode model without toxicity validates past and potential future therapeutic use.

Ellagitannins were elucidated as the active compounds in C. erectus, and displayed anti-QS activity against $P$. aeruginosa (Chapter 5). It would be valuable to assess the dose-dependance of these compounds and to test their ability to prevent infection in a nematode model system (Chapter 4; (Adonizio et al., 2008a)) and perhaps the murine model developed for halogenated furanones (Wu et al., 2004).

The medicinal plants B. buceras and C. viminalis also hold very promising antiQS activity and their chemistry should be explored. Since B. buceras is in the same family as $C$. erectus, its activity is potentially due to similar ellagitannins. Tannins in the Myrtaceae however are considerably different (Okuda et al., 1982; Yoshida et al., 2007), and may or may not be the responsible compounds in this species.

Future research into the naturally-occurring ellagitannins is also quite valuable. Ellagitannin containing fruits such as raspberry, blackberry blueberry, and grape have been popularized as nutraceuticals for their anti-oxidant and heart-health benefits (Espin et al., 2007). However, a recent study shows these fruits to have anti-QS activity in vitro (Vattem et al., 2007). In light of the research contained herein, this activity is likely due to their high ellagitannin content. The variability of ellagic and other types of tannins in 
the plant kingdom, combined with numerous opportunities for chemical modification, provides us a new resource with indispensable therapeutic potential. Subtle changes made to the structure of these tannins such as the addition or removal of functional groups could increase their efficacy. The anti-QS activity of this class of compounds is relevant not only in the attenuation of Pseudomonas infections, but in the treatment of other bacterial diseases as well.

Although a few potential modes of action have been proposed in this dissertation, exact mechanistic interactions with the QS system should at some point be resolved for ellagitannins. Tannins have been shown to interact with the bacterial cell membrane suggesting the ability of these compounds to cross the cell wall (Scalbert, 1991). Specific localization and protein binding studies could be used to gauge potential interactions with individual components of the QS system. Finally, the continued screening of medicinal plants and libraries for anti-QS activity is also of the utmost importance, as many new compounds await discovery. 


\section{BIBLIOGRAPHY}

Abraham, E. P. \& Chain, E. (1940). An enzyme from bacteria able to destroy penicillin. Nature 146, 837-838.

Abraham, E. P., Chain, E., Fletcher, C. M., Florey, H. W., Gardner, A. D., Heatley, N. G. \& Jennings, M. A. (1941). Further observations on penicillin. European Journal of Clinical Pharmacology 42, 3-9.

Adam, K., Sivropoulou, A., Kokkini, S., Lanaras, T. \& Arsenakis, M. (1998). Antifungal activities of Origanum vulgare subsp. hirtum, Mentha spicata, Lavandula angustifolia, and, Salvia fruticosa essential oils against human pathogenic fungi. Journal Agricultural Food Chemistry 46, 1739-1745.

Adonizio, A., Jr., S. M. L., Ausubel, F. M. \& Mathee, K. (2008a). Medicinal plant extracts attenuate Pseudomonas aeruginosa killing of Caenorhabditis elegans through bacterial virulence inhibition. Journal of Medical Microbiology (In Press).

Adonizio, A., Kong, K.-F. \& Mathee, K. (2008b). Inhibition of quorum sensingcontrolled virulence factor production in Pseudomonas aeruginosa by south Florida plant extracts. Antimicrobial Agents and Chemotherapy 52, 198-203.

Adonizio, A. L., Downum, K., Bennett, B. C. \& Mathee, K. (2006). Anti-quorum sensing activity of medicinal plants in southern Florida. Journal of Ethnopharmacology 103, 427435.

Albus, A., Pesci, E., Runyen-Janecky, L., West, S. \& Iglewski, B. (1997). Vfr controls quorum sensing in Pseudomonas aeruginosa. Journal of Bacteriology 179, 3928-3935.

Ambler, R. P. (1980). The structure of beta-lactamases. Philosophical Transactions of the Royal Society of London B Biological Sciences 289, 321-331.

APGII (2003). An update of the Angiosperm Phylogeny Group classification for the orders and families of flowering plants: APG II. Botanical Journal of the Linnean Society $141,399-436$.

Asif Saeed, M. \& Sabir, A. W. (2004). Antibacterial activities of some constituents from oleo-gum-resin of Commiphora mukul. Fitoterapia 75, 204-208.

Austin, D. F. (1997). Glades Indians and the plants they used. Ethnobotany of an extinct culture. The Palmetto 17, 7-11.

Austin, D. F. (2004). Florida Ethnobotany Boca Raton: CRC Press LLC. 
Balandrin, N. F., Kingkorn, A. D. \& Farnsworth, N. R. (1993).Human Medicinal Agents from Plants. In ACS Symposium Series, pp. 2-12. Edited by A. D. Kingkorn \& N. F. Balandrin.

Balick, M. J. \& Cox, P. A. (1999). Plants, People, and Culture: The Science of Ethnobotany. New York: Scientific American Library.

Bassler, B. L., Wright, M., Showalter, R. E. \& Silverman, M. R. (1993). Intercellular signalling in Vibrio harveyi: sequence and function of genes regulating expression of luminescence. Molecular Microbiology 9, 773-786.

Bauer, A. W., Kirby, W. M., Sherris, J. C. \& Turck, M. (1966). Antibiotic susceptibility testing by a standardized single disk method. American Journal of Clinical Pathology 45, 493-496.

Bauer, W. D. \& Mathesius, U. (2004). Plant responses to bacterial quorum sensing signals. Current Opinion in Plant Biology 7, 429-433.

Bauer, W. D., Tepletski, M. (2001). Can plants manipulate bacterial quorum sensing? Australian Journal of Plant Physiology 28, 913-921.

Bennett, B. C., Baker, M. A. \& Gomez-Andrade, P. (2002). Ethnobotany of the Shuar of Eastern Ecuador. Advances in Economics Botany 14, 1-299.

Benyhe, S. (1994). Morphine: New aspects in the study of an ancient compound. Life Sciences 55, 969-979.

Bergey (2001). Bergey's manual of systematic bacteriology, 2nd edition edn. New York: Springer-Verlag.

Blumenthal, M., Goldberg, A. \& Brinckmann, J. (2000).Herbal Medicine: Expanded Commission E Monographs, pp. 519. Newton, MA: Integrative Medicine Communications.

Bodey, G. P., Bolivar, R., Fainstein, V. \& Jadeja, L. (1983). Infections caused by Pseudomonas aeruginosa. Reviews of Infectious Diseases 5, 279-313.

Bradford, M. M. (1976). A rapid and sensitive method for the quantitation of microgram quantities of protein utilizing the principle of protein-dye binding Analytical Biochemistry 72, 248-254.

Burkhill, H. M. (1985). The useful plants of west tropical Africa. London: Kew: Royal Botanic Gardens. 
Bush, K., Jacoby, G. A. \& Medeiros, A. A. (1995). A functional classification scheme for beta-lactamases and its correlation with molecular structure. Antimicrobial Agents and Chemotherapy 39, 1211-1233.

Bussey, H. I., Hawkins, D. W., Gaspard, J. J. \& Walsh, R. A. (1988). A comparative trial of digoxin and digitoxin in the treatment of congestive heart failure. Pharmacotherapy 8 , $235-240$.

Bycroft, B. W. \& Shute, R. E. (1985). The molecular basis for the mode of action of betalactam antibiotics and mechanisms of resistance. Pharmaceutical Research 2, 3-14.

Calfee, M. W., Coleman, J. P. \& Pesci, E. C. (2001). Interference with Pseudomonas quinolone signal synthesis inhibits virulence factor expression by Pseudomonas aeruginosa. Proceedings of the National Academy of Sciences of the United States of America 98, 11633-11637.

Carson, C. F. \& Riley, T. V. (1995). Antimicrobial activity of the major components of the essential oil of Melaleuca alternifolia. Journal of Applied Bacteriology 78, 264-269.

Carson, C. F., Riley, T. V. \& Cookson, B. D. (1998). Efficacy and safety of tea tree oil as a topical antimicrobial agent. Journal of Hospital Infection 40, 175-178.

Centers for Disease Control and Prevention, H. I. P. (2004). National Nosocomial Infections Surveillance (NNIS) report, data summary from January 1992-June 2004, issued October 2004: A report from the NNIS System. American Journal of Infection Control 32, 470-485.

Cha, C. G., P., Chen, Y.C., Shaw, P.D., Farrand, S.K. (1998). Production of acylhomoserine lactone quorum-sensing signals by gram-negative plant-associated bacteria. Molecular Plant Microbe Interactions 11, 1119-1129.

Chami, F., Chami, N., Bennis, S., Trouillas, J. \& Remmal, A. (2004). Evaluation of carvacrol and eugenol as prophylaxis and treatment of vaginal candidiasis in an immunosuppressed rat model. Journal of Antimicrobial Chemotherapy 54, 909-914.

Chun, S.-S., Vattem, D. A., Lin, Y.-T. \& Shetty, K. (2005). Phenolic antioxidants from clonal oregano (Origanum vulgare) with antimicrobial activity against Helicobacter pylori. Process Biochemistry 40, 809-816.

Clark, D. J. \& Maaloe, O. (1967). DNA replication and the division cycle in Escherichia coli. Journal of Molecular Biology 23, 99-112.

Clark, W. B., Brook, I., Bianki, D. \& Thompson, D. H. (1997). Microbiology of otitis externa. Otolaryngology - Head and Neck Surgery 116, 23-25. 
Costerton, J. W., Lewandowski, Z., Caldwell, D. E., Korber, D. R. \& Lappin-Scott, H. M. (1995). Microbial biofilms. Annual Review of Microbiology 49, 711-745.

Costerton, J. W., Lewandowski, Z., Caldwell, D.E., Korber, D.R., Lappin-Scott, H.M. (1995). Microbial biofilms. Annual Review of Microbiology 49, 711-745.

Cowan, M. M. (1999). Plant products as antimicrobial agents. Clinical Microbiology Reviews 12, 564-582.

Cox, C. D. \& Adams, P. (1985). Siderophore activity of pyoverdin for Pseudomonas aeruginosa. Infection and Immunity 48, 130-138.

Cumberbatch, A. (2002). Characterization of the anti-quorum sensing activity exhibited by marine macroalgae of South Florida. In Department of Biological Sciences. Miami: Florida International University.

Davies, D. G., Parsek, M. R., Pearson, J. P., Iglewski, B. H., Costerton, J. W. \& Greenberg, E. P. (1998). The involvement of cell-to-cell signals in the development of a bacterial biofilm. Science 280, 295-298.

Debler, E. W., Kaufmann, G. F., Kirchdoerfer, R. N., Mee, J. M., Janda, K. D. \& Wilson, I. A. (2007). Crystal structures of a quorum-quenching antibody. Journal of Molecular Biology 368, 1392-1402.

Dewick, P. M. (2002). Medicinal Natural Products: A Biosynthetic Approach, Second edn. New York: John Wiley and Sons.

Dong, Y. H., Wang, L. H., Xu, J. L., Zhang, H. B., Zhang, X. F. \& Zhang, L. H. (2001). Quenching quorum-sensing-dependent bacterial infection by an $N$-acyl homoserine lactonase. Nature 411, 813-817.

Dong, Y. H. \& Zhang, L. H. (2005). Quorum sensing and quorum-quenching enzymes. Journal of Microbiology 43 Spec No, 101-109.

Donlan, R. M. \& Costerton, J. W. (2002). Biofilms: survival mechanisms of clinically relevant microorganisms. Clinical Microbiology Reviews 15, 167-193.

Duke, J. A. (1985). CRC Handbook of Medicinal Herbs. Boca Raton, FL: CRC Press.

Eberhard, A., Burlingame, A. L., Eberhard, C., Kenyon, G. L., Nealson, K. H. \& Oppenheimer, N. J. (1981). Structural identification of autoinducer of Photobacterium fischeri luciferase. Biochemistry 163, 2444-2449.

Espin, J. C., Garcia-Conesa, M. T. \& Tomas-Barberan, F. A. (2007). Nutraceuticals: facts and fiction. Phytochemistry 68, 2986-3008. 
Fairbrother, R. W. \& Taylor, G. (1961). Sodium methicillin in routine therapy. Lancet 1, 473-476.

Flanders, S. A., Collard, H. R. \& Saint, S. (2006). Nosocomial pneumonia: state of the science. American Journal of Infection Control 34, 84-93.

Foca, M. D. (2002). Pseudomonas aeruginosa infections in the neonatal intensive care unit. Seminars in Perinatology 26, 332-339.

Frizzell, R. A. (1999). Physiology of Cystic Fibrosis. New Haven, CT: American Physiology Society.

Fuqua, C. \& Greenberg, E. P. (1998). Self perception in bacteria: quorum sensing with acylated homoserine lactones. Current Opinion in Microbiology 1, 183-189.

Fuqua, C. \& Greenberg, E. P. (2002). Listening in on bacteria: acyl-homoserine lactone signalling. Nature Reviews Molecular and Cellular Biology 3, 685-695.

Fuqua, W. C., Winans, S. C. \& Greenberg, E. P. (1994). Quorum sensing in bacteria: the LuxR-LuxI family of cell density-responsive transcriptional regulators. Journal of Bacteriology 176, 269-275.

Gallagher, L. A. \& Manoil, C. (2001). Pseudomonas aeruginosa PAO1 kills Caenorhabditis elegans by cyanide poisoning. Journal of Bacteriology 183, 6207-6214.

Galleni, M., Lindberg, F., Normark, S., Cole, S., Honore, N., Joris, B. \& Frere, J. M. (1988). Sequence and comparative analysis of three Enterobacter cloacae ampC betalactamase genes and their products. Biochemical Journal 250, 753-760.

Gambello, M. J., Iglewski, B.H (1991). Cloning and characterization of the Pseudomonas aeruginosa lasR gene, a transcriptional activator of elastase expression. Journal of Bacteriology 179, 3000-3009.

Garg, S. C. \& Kasera, H. L. (1982). Antihelminthic activity of the essential oil of Callistemon viminalis. Fitoterapia 53, 179-181.

Givskov, M., de Nys, R., Manefield, M., Gram, L., Maximilien, R., Eberl, L., Molin, S., Steinberg, P. D. \& Kjelleberg, S. (1996). Eukaryotic interference with homoserine lactone-mediated prokaryotic signalling. Journal of Bacteriology 178, 6618-6622.

Goggin, J. M. \& Sturtevant, W. C. (1964). The Calusa: A Stratified, Nonagricultural Society (with Notes on Sibling Marriage). In Explorations in Cultural Anthropology: Essays in Honor of George Peter Murdock, pp. 179-219. Edited by W. H. Goodenough. New York: McGraw-Hill Book Company. 
Gurevitch, J., Scheiner, S. M. \& Fox, G. A. (2002). The Ecology of Plants. Sunderland, MA: Sinauer Associates Inc.

Halcon, L. \& Milkus, K. (2004). Staphylococcus aureus and wounds: A review of tea tree oil as a promising antimicrobial. American Journal of Infection Control 32, 402-408.

Harshberger, J. W. (1896). The purposes of ethno-botany. Botanical Gazette 21, 146-154.

Hastings, J. W. \& Greenberg, E. P. (1999). Quorum sensing: the explanation of a curious phenomenon reveals a common characteristic of bacteria. Journal of Bacteriology 181, 2667-2668.

Heijden, R., Jacobs, D. I., Snoeijer, W., Hallard, D. \& Verpoorte, R. (2004). The Catharanthus alkaloids: pharmacognosy and biotechnology. Current Medicinal Chemistry 11, 607-628.

Henke, J. M. \& Bassler, B. L. (2004). Bacterial social engagements. Trends in Cell Biology 14, 648-656.

Hentzer, M., Reidel, K., Rasmussen, T. B. \& other authors (2002). Inhibition of quorum sensing in Pseudomonas aeruginosa biofilm bacteria by a halogenated furanone compound. Microbiology 148, 87-102.

Hentzer, M. \& Givskov, M. (2003a). Pharmacological inhibition of quorum sensing for the treatment of chronic bacterial infections. Journal of Clinical Investigation 112, 13001307.

Hentzer, M. \& Givskov, M. (2003b). Pharmacological inhibition of quorum sensing for the treatment of chronic bacterial infections. Journal of Clinical Investigation 112, 13001307.

Hentzer, M., Wu, H., Andersen, J. B. \& other authors (2003). Attenuation of Pseudomonas aeruginosa virulence by quorum sensing inhibitors. The EMBO Journal 22, 3803-3815.

Hersch-Martinez, P., Leanos-Miranda, B. E. \& Solorzano-Santos, F. (2005). Antibacterial effects of commercial essential oils over locally prevalent pathogenic strains in Mexico. Fitoterapia 76, 453-457.

Hoang, T. T., Sullivan, S. A., Cusick, J. K. \& Schweizer, H. P. (2002). \{beta\}-Ketoacyl acyl carrier protein reductase (FabG) activity of the fatty acid biosynthetic pathway is a determining factor of 3-oxo-homoserine lactone acyl chain lengths. Microbiology 148, 3849-3856. 
Hoiby, N. (1993). Antibiotic therapy for chronic infection of Pseudomonas in the lung. Annual Review of Medicine 44, 1-10.

Holden, M. T. G., Chhabra, S.R., de Nys, R., Stead, P., Balnton, N.J., Hill, P.J., Manefield, M., Kumar, N., Maurice, L., England, D., Rice, S., Glvskov, M., Salmond, G.P.C., Stewart, G.S.A.B., Bycroft, B.W., Kjelleberg, S., Williams, P. (1999). Quorumsensing cross talk: isolation and chemical characterization of cyclic dipeptides from Pseudomonas aeruginosa and other Gram-negative bacteria. Molecular Microbiology 33, 1254-1266.

Holloway, B. W. \& Morgan, A. F. (1986). Genome organization in Pseudomonas. Annual Review of Microbiology 40, 79-105.

Hooper, D. C. (1999). Mode of action of fluoroquinolones. Drugs 58, 6-10.

Hoyle, B. \& Costerton, J. W. (1991). Bacterial resistance to antibiotics: the role of biofilms. Progress in Drug Research 37, 91-105.

Huber, B., Eberl, L., Feucht, W. \& Polster, J. (2004). Influence of polyphenols on bacterial biofilm formation and quorum-sensing. Zeitschrift fur Naturforschung Section C, Biosciences 58, 879-884.

Irvine, F. R. (1961). Woody Plants of Ghana: Oxford University Press, London.

Jacques, P., Ongena, M., Bernard, F., Fuchs, R., Budzikiewicz, H. \& Thonart, P. (2003). Fluorescent Pseudomonas mainly produce the dihydroform of pyoverdine at low specific growth rate. Letters in Applied Microbiology 36, 259-262.

Judd, W. S., Campbell, C. S., Kellogg, E. A., Stevens, P. F. \& Donoghue, M. J. (2002). Plant Systematics: A Phylogenetic Approach, Second Ed. edn. Sunderland, MA: Sinauer Associates Inc.

Kessler, E. (1995). Beta-lytic endopeptidases. Methods in Enzymology 248, 740-756.

Khafagi, I. K. \& Dewedar, A. (2000). The efficiency of random versus ethno-directed research in the evaluation of Sinai medicinal plants for bioactive compounds. Journal of Ethnopharmacology 71, 365-376.

Khanbabaee, K. \& van Ree, T. (2001). Tannins: classification and definition. Natural Product Reports 18, 641-649.

Kharazmi, A. (1989). Interactions of Pseudomonas aeruginosa proteases with the cells of the immune system. Antibiotics and Chemotherapy 42, 42-49. 
Krcmery, V., Koprnova, J., Gogova, M., Grey, E. \& Korcova, J. (2006). Pseudomonas aeruginosa bacteraemia in cancer patients. Journal of Infection 52, 461-463.

Kremsner, P. G., Winkler, S., Brandts, C., Neifer, S., Bienzle, U. \& Graninger, W. (1994). Clindamycin in combination with chloroquine or quinine is an effective therapy for uncomplicated Plasmodium falciparum malaria in children from Gabon. Journal of Infectious Diseases 169.

Lacassie, E., Marquet, P., Martin-Dupont, S., Gaulier, J. M. \& Lachatre, G. (2000). A non-fatal case of intoxication with foxglove, documented by means of liquid chromatography-electrospray-mass spectrometry. Journal of Forensic Sciences 45, 11541158.

Lewis, K. \& Ausubel, F. M. (2006). Prospects for plant-derived antibacterials. Nature Biotechnology 24, 1504-1507.

Liberati, N. T., Urbach, J. M., Miyata, S., Lee, D. G., Drenkard, E., Wu, G., Villanueva, J., Wei, T. \& Ausubel, F. M. (2006). An ordered, nonredundant library of Pseudomonas aeruginosa strain PA14 transposon insertion mutants. Proceedings of the National Academy of Sciences of the United States of America 103, 2833-2838.

Lichstein, H. C. \& van de Sand, V. F. (1945). Violacein, an antibiotic pigment produced by Chromobacterium violaceum. Journal of Infectious Disease 76, 47-51.

Lodge, J. M., Minchin, S. D., Piddock, L. J. \& Busby, J. W. (1990). Cloning, sequencing and analysis of the structural gene and regulatory region of the Pseudomonas aeruginosa chromosomal ampC beta-lactamase. Biochemical Journal 272, 627-631.

Luo, Z.-Q., Su, S. \& Farrand, S. K. (2003). In situ activation of the quorum-sensing transcription factor TraR by cognate and noncognate acyl-homoserine lactone ligands: kinetics and consequences. Journal of Bacteriology 185, 5665-5672.

Lyczak, J. B., Cannon, C. L. \& Pier, G. B. (2002). Lung infections associated with cystic fibrosis. Clinical Microbiology Reviews 15, 194-222.

Madersbacher, S., Ponholzer, A., Berger, I. \& Marszalek, M. (2007). Medical Management of BPH: Role of Plant Extracts. EAU-EBU Update Series 5, 197-205.

Makemson, J., Eberhard, A. \& Mathee, K. (2006). Simple electrospray mass spectrometry detection of acylhomoserine lactones. Luminescence 21, 1-6.

Manefield, M., de Nys, R., Kumar, N., Read, R., Givskov, M., Steinberg, P. \& Kjelleberg, S. (1999). Evidence that halogenated furanones from Delisea pulchra inhibit acylated homoserine lactone (AHL)-mediated gene expression by displacing the AHL signal from its receptor protein. Microbiology 145, 283-291. 
Manefield, M., Rasmussen, T. B., Henzter, M., Andersen, J. B., Steinberg, P., Kjelleberg, S. \& Givskov, M. (2002). Halogenated furanones inhibit quorum sensing through accelerated LuxR turnover. Microbiology 148, 1119-1127.

Marcet, B. \& Boeynaems, J.-M. (2006). Relationships between cystic fibrosis transmembrane conductance regulator, extracellular nucleotides and cystic fibrosis. Pharmacology \& Therapeutics 112, 719-732.

Martinelli, D., Grossmann, G., Sequin, U., Brandl, H. \& Bachofen, R. (2004). Effects of natural and chemically synthesized furanones on quorum sensing in Chromobacterium violaceum. BMC Microbiology 4, 25.

Matagne, A., Misselyn-Bauduin, A. M., Joris, B., Erpicum, T., Granier, B. \& Frere, J. M. (1990). The diversity of the catalytic properties of class A beta-lactamases. Biochemical Journal 265, 131-146.

Mathee, K., McPherson, C. \& Ohman, D. (1997). Posttranslational control of the algT (algU)-encoded sigma22 for expression of the alginate regulon in Pseudomonas aeruginosa and localization of its antagonist proteins MucA and MucB (AlgN). Journal of Bacteriology 179, 3711-3720.

McClean, R. J. C., Pierson, I., Leland S. \& Fuqua, C. (2004). A simple screening protocol for the identification of quorum signal antagonists. Journal of Microbiological Methods $58,351-360$.

McGoun, W. E. (1993). Prehistoric People of South Florida. Tuscaloosa: University of Alabama Press.

McKnight, S. L., Iglewski, B. H. \& Pesci, E. C. (2000). The Pseudomonas quinolone signal regulates $r$ hl quorum sensing in Pseudomonas aeruginosa. Journal of Bacteriology $182,2702-2708$.

Melendez, E. N. (1982). Plantas medicinales de Puerto Rico : folklore y fundamentos científicos. Río Piedras, P.R.: Editorial de la Unversidad de Puerto Rico.

Mendez, C. \& Salas, J. A. (2001). The role of ABC transporters in antibiotic-producing organisms: drug secretion and resistance mechanisms. Research in Microbiology 152, $341-350$.

Messager, S., Hammer, K. A., Carson, C. F. \& Riley, T. V. (2005). Assessment of the antibacterial activity of tea tree oil using the European EN 1276 and EN 12054 standard suspension tests. Journal of Hospital Infection 59, 113-125.

Meyer, J., Neely, A., Stintzi, A., Georges, C. \& Holder, I. (1996). Pyoverdin is essential for virulence of Pseudomonas aeruginosa. Infection and Immunity 64, 518-523. 
Meynard, J.-L., Barbut, F., Guiguet, M. \& other authors (1999). Pseudomonas aeruginosa infection in human immunodeficiency virus infected patients. Journal of Infection 38, 176-181.

Milanich, J. T. (1995). Florida Indians and the Invasion from Europe. Gainesville: University Press of Florida

Moerman, D. (1998). Native American Ethnobotany. Oregon: Timber Press.

Morihara, K. \& Homma, J. Y. (1985). Pseudomonas Proteases. In Bacterial Enzymes and Virulence, pp. 41-79. Edited by I. Holder. Boca Raton, FL: CRC Press, Inc.

Morton, J. F. (1981). Atlas of Medicinal Plants of Middle America: Bahamas to Yucatan. Springfield, IL: Charles C. Thomas.

Naas, T. \& Nordmann, P. (1999). OXA-type beta-lactamases. Current Pharmaceutical Design 5, 865-879.

Nealson, K. H., Platt, T. \& Hastings, J. W. (1970). Cellular control of the synthesis and activity of the bacterial luminescent system. Journal of Bacteriology 104, 313-322.

Nellis, D. W. (1994). Seashore Plants of South Florida and the Caribbean. Sarasota, FL: Pineapple Press.

Nicolle, L. E. (2002). Resistant pathogens in urinary tract infections. Journal of the American Geriatrics Society 50, S230-S235.

O'Toole, G. A. \& Kolter, R. (1998). Initiation of biofilm formation in Pseudomonas fluorescens WCS365 proceeds via multiple, convergent signaling pathways: a genetic analysis. Molecular Microbiology 28, 449-461.

Ohman, D. E., Cryz, S. J. \& Iglewski, B. H. (1980). Isolation and characterization of a Pseudomonas aeruginosa PAO mutant that produces altered elastase. Journal of Bacteriology 142, 836-842.

Okuda, T., Yoshida, T. \& Hatano, T. (1993). Classification of oligomeric hydrolysable tannins and specificity of their occurrence in plants. Phytochemistry 32, 507-521.

Okuda, T., Yoshida, T. \& Hatano, T. (2000). Correlation of oxidative transformations of hydrolyzable tannins and plant evolution. Phytochemistry 55, 513-529.

Oszmianski, J., Ramos, T. \& Bourzeix, M. (1988). Fractionation of phenolic compounds in red wine. American Journal of Enology and Viticulture 39, 259-262. 
Park, H., Jang, C. H., Cho, Y. B. \& Choi, C. H. (2007). Antibacterial effect of tea-tree oil on methicillin-resistant Staphylococcus aureus biofilm formation of the tympanostomy tube: an in vitro study. In Vivo 21, 1027-1030.

Pesci, E., Pearson, J., Seed, P. \& Iglewski, B. (1997). Regulation of las and rhl quorum sensing in Pseudomonas aeruginosa. Journal of Bacteriology 179, 3127-3132.

Pesci, E. C., Milbank, J. B. J., Pearson, J. P., McKnight, S., Kende, A. S., Greenberg, E. P. \& Iglewski, B. H. (1999). Quinolone signaling in the cell-to-cell communication system of Pseudomonas aeruginosa. Proceedings of the National Academy of Sciences of the United States of America 96, 11229-11234.

Pessi, G. \& Haas, D. (2000). Transcriptional control of the hydrogen cyanide biosynthetic genes hcn $\mathrm{ABC}$ by the anaerobic regulator ANR and the quorum-sensing regulators LasR and RhlR in Pseudomonas aeruginosa. Journal of Bacteriology 182, 6940-6949.

Poirel, L., Weldhagen, G.F., De Champs, C., Nordmann, P. (2002). A nosocomial outbreak of Pseudomonas aeruginosa isolates expressing the extended-spectrum betalactamase GES-2 in South Africa. Journal of Antimicrobial Chemotherapy 49, 561-565.

Poole, K. \& Srikumar, R. (2001). Multidrug efflux in Pseudomonas aeruginosa: components, mechanisms and clinical significance. Current Topics in Medicinal Chemistry 1, 59-71.

Quideau, S. \& Feldman, K. S. (1996). Ellagitannin chemistry. Chemical Reviews 96, 475504.

Rahme, L. G., Stevens, E. J., Wolfort, S. F., Shao, J., Tompkins, R. G. \& Ausubel, F. M. (1995). Common virulence factors for bacterial pathogenicity in plants and animals. Science 268, 1899-1902.

Rasmussen, T. B., Manefield, M., Andersen, J. B., Eberl, L., Anthoni, U., Christophersen, C., Steinberg, P., Kjelleberg, S. \& Givskov, M. (2000). How Delisea pulchra furanones affect quorum sensing and swarming motility in Serratia liquefaciens MG1. Microbiology 146, 3237-3244.

Rasmussen, T. B., Bjarnsholt, T., Skindersoe, M. E., Hentzer, M., Kristoffersen, P., Kote, M., Nielsen, J., Eberl, L. \& Givskov, M. (2005). Screening for quorum-sensing inhibitors (QSI) by use of a novel genetic system, the QSI Selector. Journal of Bacteriology 187, $1799-1814$

Registry (2005). Patient Registry 2004 Annual Report. Bethesda, Maryland: Cystic Fibrosis Foundation. 
Regules, J. A., Glasser, J. S., Wolf, S. E., Hospenthal, D. R. \& Murray, C. K. (2007). Endocarditis in burn patients: clinical and diagnostic considerations. Burns.

Reimmann, C., Beyeler, M., Latifi, A., Winteler, H., Foglino, M., Lazdunski, A. \& Haas, D. (1997). The global activator GacA of Pseudomonas aeruginosa PAO positively controls the production of the autoinducer N-butyryl-homoserine lactone and the formation of the virulence factors pyocyanin, cyanide, and lipase. Molecular Microbiology 24, 309-319.

Reimmann, C., Ginet, N., Michel, L. \& other authors (2002). Genetically programmed autoinducer destruction reduces virulence gene expression and swarming motility in Pseudomonas aeruginosa PAO1. Microbiology 148, 923-932.

Ren, D., Zuo, R. \& Wood, T. K. (2005). Quorum-sensing antagonist (5Z)-4-bromo-5(bromomethylene)-3-butyl-2(5H)-furanone influences siderophore biosynthesis in Pseudomonas putida and Pseudomonas aeruginosa. Applied Microbiology and Biotechnology 66, 689-695.

Robertson, D. M., Petroll, W. M., Jester, J. V. \& Cavanagh, H. D. (2007). Current concepts: contact lens related Pseudomonas keratitis. Contact Lens and Anterior Eye 30, 94-107.

Rodelas, B., Lithgow, J. K., Wisniewski-Dye, F., Hardman, A., Wilkinson, A., Economou, A., Williams, P. \& Downie, J. A. (1999). Analysis of quorum-sensingdependent control of rhizosphere-expressed (rhi) genes in Rhizobium leguminosarum bv. viciae. Journal of Bacteriology 181, 3816-3823.

Rodrigues, M. R., Krause, L. C., Caramo, E. B., dos Santos, J. G., Dariva, C. \& Vladimir de Oliveira, J. (2004). Chemical composition and extraction yield of the extract of Origanum vulgare obtained from sub- and supercritical CO2. Journal of Agricultural and Food Chemistry 52, 3042-3047.

Roland, P. S. (2002). Chronic suppurative otitis media: a clinical overview. Ear, Nose, and Throat Journal 81, S8-S10.

Rowe, M. I., Reblock, K. K., Kurkchubasche, A. G. \& Healey, P. J. (1994). Necrotizing enterocolitis in the extremely low birth weight infant. Journal of Pediatric Surgery 29, 987-991.

Sakar, M. K., Sohertoglu, D., Ozalp, M., Ekizoglu, M., Piacente, S. \& Pizza, C. (2005). Polyphenolic compounds and antimicrobial activity of Quercus aucheri leaves. Turkish Journal of Chemistry 29, 555-559.

Sanders, C. C. (1987). Chromosomal cephalosporinases responsible for multiple resistance to newer beta-lactam antibiotics. Annual Review of Microbiology 41, 573-593. 
Sapico, F. L. \& Montgomerie, J. Z. (1990). Vertebral osteomyelitis. Infectious Disease Clinics of North America 4, 539-550.

Satchell, A. C., Saurajen, A., Bell, C. \& Barnetson, R. S. (2002). Treatment of dandruff with 5\% tea tree oil shampoo. Journal of the American Academy of Dermatology 47, 852855.

Scalbert, A. (1991). Antimicrobial properties of tannins. Phytochemistry 30, 3875-3883.

Schauder, S. \& Bassler, B. L. (2001). The languages of bacteria. Genes and Development $15,1468-1480$.

Schuster, M. \& Greenberg, E. P. (2006). A network of networks: quorum-sensing gene regulation in Pseudomonas aeruginosa. International Journal of Medical Microbiology 296, 73-81.

Sheppard, D. N. \& Welsh, M. J. (1999). Structure and function of the CFTR chloride channel. Physiological Reviews 79, S23-45.

Smith, R. S. \& Iglewski, B. H. (2003a). Pseudomonas aeruginosa quorum sensing as a potential antimicrobial target. Journal of Clinical Investigation 112, 1460-1465.

Smith, R. S. \& Iglewski, B. H. (2003b). Pseudomonas aeruginosa quorum-sensing systems and virulence. Current Opinion in Microbiology 6, 56-60.

Smith, R. S. \& Iglewski, B. H. (2003c). Pseudomonas aeruginosa quorum sensing as a potential antimicrobial target. Journal of Clinical Investigation 112, 1460-1465.

Stevens, A. \& Greenberg, E. (1997). Quorum sensing in Vibrio fischeri: essential elements for activation of the luminescence genes. Journal of Bacteriology 179, 557-562.

Sun, B., Leandro, M. C., de Freitas, V. \& Spranger, M. I. (2006). Fractionation of red wine polyphenols by solid-phase extraction and liquid chromatography. Journal of Chromatography A 1128, 27-38.

Surette, M. G. \& Bassler, B. L. (1998). Quorum sensing in Escherichia coli and Salmonella typhimurium. Proceedings of the National Academy of Sciences of the United States of America 95, 7046-7050.

Tan, M. W., Mahajan-Miklos, S. \& Ausubel, F. M. (1999). Killing of Caenorhabditis elegans by Pseudomonas aeruginosa used to model mammalian bacterial pathogenesis. Proceedings of the National Academy of Sciences of the United States of America 96, 715-720. 
Tang, H. R. \& Hancock, R. A. (1995). Studies of vegetable tannins. Complete structural determination of two chestnut tannins-vescalagin and castalagin using nuclear magnetic resonance spectroscopic methods. Journal of the Society of Leather Technologists and Chemists 79, 181-187.

Teplitski, M., Robinson, J. B. \& Bauer, W. D. (2000). Plants secrete substances that mimic bacterial $\mathrm{N}$-acyl homoserine lactone signal activities and affect population densitydependent behaviors in associated bacteria. Molecular Plant-Microbe Interactions 13, 637-648.

Throup, J., Winson, M. K., Bainton, N. J., Bycroft, B. W., Williams, P. \& Stewart, G. S. A. B. (1995). Signalling in bacteria beyond bioluminescence. In Bioluminescence and chemiluminescence: Fundamentals and applied aspects. Edited by A. Campbell, L. Kricka \& P. Stanley. Chichester: Wiley.

Tsai, M. J., Teng, C. J., Teng, R. J., Lee, P. I. \& Chang, M. H. (1996). Necrotizing bowel lesions complicated by Pseudomonas septicaemia in previously healthy infants. European Journal of Pediatrics 155, 216-218.

Tyler, V. E., Brady, L. R. \& Robbers, J. E. (1981). Pharmacognosy, 8th edn. Philadelphia: Lea and Febiger.

Van Delden, C. \& Iglewski, B. H. (1998). Cell-to-cell signaling and Pseudomonas aeruginosa infections. Emerging Infectious Diseases 4, 551-560.

Vasil, M. L. (1986). Pseudomonas aeruginosa: biology, mechanisms of virulence, epidemiology. Journal of Pediatrics 108, 800-805.

Vattem, D. A., Mihalik, K., Crixell, S. H. \& McLean, R. J. C. (2007). Dietary phytochemicals as quorum sensing inhibitors. Fitoterapia 78, 302-310.

Venturi, V. (2006). Regulation of quorum sensing in Pseudomonas. FEMS Microbiology Reviews 30, 274-291.

Von Bodman, S. B., Bauer, W. D. \& Coplin, D. L. (2003). Quorum sensing in plantpathogenic bacteria. Annual Review of Phytopathology 41, 455-482.

Wall, M. E., Wani, M. C., Cook, C. E., Palmer, K. H., McPhail, A. T. \& Sim, G. A. (1966). Plant antitumor agents I. The isolation and structure of camptothecin, a novel alkaloidal leukemia and tumor inhibitor from Camtotheca acuminata. Journal of the American Chemical Society 88, 3388-3890.

Walsh, T. R., Toleman, M. A., Poirel, L. \& Nordmann, P. (2005). Metallo-betalactamases: the quiet before the storm? Clinical Microbiology Reviews 18, 306-325. 
Wani, M. C., Taylor, H. L., Wall, M. E., Coggon, P. \& McPhail, A. T. (1971). Plant antitumor agents VI. The isolation and structure of Taxol a novel antileukemic and antitumor agent from Taxus brevifolia. Journal of the American Chemical Society 93, 2325-2327.

Watanabe, M., Iyobe, S., Inoue, M. \& Mitsuhashi, S. (1991). Transferable imipenem resistance in Pseudomonas aeruginosa. Antimicrobial Agents and Chemotherapy 35, 147-151.

Weldhagen, G. F., Poirel, L. \& Nordmann, P. (2003). Ambler class A extended-spectrum beta-lactamases in Pseudomonas aeruginosa: novel developments and clinical impact. Antimicrobial Agents and Chemotherapy 47, 2385-2392.

Whitehead, N. A., Barnard, A. M., Slater, H., Simpson, N. J. \& Salmond, G. P. (2001a). Quorum-sensing in Gram-negative bacteria. FEMS Microbiology Reviews 25, 365-404.

Whitehead, N. A., Welch, M. \& Salmond, G. P. C. (2001b). Silencing the majority. Nature Biotechnology 19, 735-736.

Willsie, A., Mathee, K. (2000). Screening South Florida waters for inhibitors of acylatedhomoserine lactones. Masters Thesis. Florida International University. (Unpublished).

Wu, H., Song, Z., Hentzer, M., Andersen, J. B., Molin, S., Givskov, M. \& Hoiby, N. (2004). Synthetic furanones inhibit quorum-sensing and enhance bacterial clearance in Pseudomonas aeruginosa lung infection in mice. Journal of Antimicrobial Chemotherapy 53, 1054-1061.

Wunderlin, R. P. (1982). Guide to the Vascular Plants of Central Florida. Tampa. 472 p.: University Presses of Florida, University of South Florida.

Wunderlin, R. P. \& Hansen, B. F. (2004). Atlas of Florida Vascular Plants (http://www.plantatlas.usf.edu/). [S. M. Landry and K. N. Campbell (application development), Florida Center for Community Design and Research.]. Institute for Systematic Botany, University of South Florida. Tampa.

Wunderlin, R. P. \& Hansen, B. F. (2006). Atlas of Florida Vascular Plants (http://www.plantatlas.usf.edu/). [S. M. Landry and K. N. Campbell (application development), Florida Center for Community Design and Research.]. Institute for Systematic Botany, University of South Florida. Tampa.

Yegorov, D. Y., Kozlov, A. V., Azizova, O. A. \& Vladimirov, Y. A. (1993). Simultaneous determination of $\mathrm{Fe}(\mathrm{III})$ and $\mathrm{Fe}(\mathrm{II})$ in water solutions and tissue homogenates using desferal and 1,10-phenanthroline. Free Radical Biology \& Medicine $15,565-574$. 
Yoshimura, F. \& Nikaido, H. (1982). Permeability of Pseudomonas aeruginosa outer membrane to hydrophilic solutes. Journal of Bacteriology 152, 636-642.

Yoshimura, F., Zalman, L. S. \& Nikaido, H. (1983). Purification and properties of Pseudomonas aeruginosa porin. Journal of Biological Chemistry 258, 2308-2314.

Yu, Y., Cheng, A. S., Wang, L., Dunne, W. M. \& Bayliss, S. J. (2007). Hot tub folliculitis or hot hand-foot syndrome caused by Pseudomonas aeruginosa. Journal of the American Academy of Dermatology 57, 596-600.

Zhang, L. H. \& Dong, Y. H. (2004). Quorum sensing and signal interference: diverse implications. Molecular Microbiology 53, 1563-1571.

Zhentian, L., Jervis, J. \& Helm, R. F. (1999). C-Glycosidic ellagitannins from white oak heartwood and callus tissues. Phytochemistry 51, 751-756.

Zhu, M., David Phillipson, J., Greengrass, P. M., Bowery, N. E. \& Cai, Y. (1997). Plant polyphenols: Biologically active compounds or non-selective binders to protein? Phytochemistry 44, 441-447. 
APPENDIX

NMR STRUCTURAL DATA FOR ISOLATED ELLAGITANNINS

Vescalagin:

${ }^{1} \mathrm{H}$ NMR (600 MHz; D2O acidified with d-TFA): $\delta 4.20$ [d, 1H, J=12.7 Hz, H-6], 4.73 [dd, 1H, J=1.0, $6.8 \mathrm{~Hz}, \mathrm{H}-3$ ] , 4.90 [dd, 1H, J=2.4, $13.2 \mathrm{~Hz}, \mathrm{H}-6$ ], 4.95 [d, 1H, J=2.1 Hz, $\mathrm{H}-1$ ], 5.15 [t, 1H, J=7.1 Hz, H-4], 5.36 [t, 1H, J=1.6 Hz, H-2], 5.55 [d, 1H, J=7.3 Hz, H5], 6.73 [s, 1H, H-2(IV)], 6.86 [s, 1H, H-6(V)], 6.95 [s, 1H, H-6(III)]

${ }^{1} \mathrm{H}$ NMR (600 MHz; [D $\mathrm{D}_{6}$-acetone: $\mathrm{D}_{2} \mathrm{O}$ 8:2): $\delta 4.01$ [d, 1H, J=12.6 Hz, H-6], 4.48 [d, 1H, $\mathrm{J}=7.3 \mathrm{~Hz}, \mathrm{H}-3$ ] , 4.83 [d, 1H, J=2.1 Hz, H-1], 4.95 [dd, 1H, J=1.9, $13.1 \mathrm{~Hz}, \mathrm{H}-6$ ], 5.11 [t, $1 \mathrm{H}, \mathrm{J}=7.3 \mathrm{~Hz}, \mathrm{H}-4], 5.20$ [brs, $1 \mathrm{H}, \mathrm{H}-2], 5.55$ [d, 1H, J=7.5 Hz, H-5], 6.57 [s, 1H], 6.72 $[\mathrm{s}, 1 \mathrm{H}], 6.73[\mathrm{~s}, 1 \mathrm{H}]$

${ }^{13} \mathrm{C}$ NMR (151 MHz; D2O acidified with d-TFA; partial assignment based on HMQC and HMBC correlations): $\delta 63.8$ [C-1], 65.5 [C-6], 68.2 [C-3], 68.7 [C-4], 70.9 [C-5], 77.3 [C-2], 107.0 [C-2(IV)], 108.8 [C-6(V)], 109.3 [C-6(III)], 113.5 [C-2(III)], 113.5 [C6(IV)], 115.2 [C-2(V)], 117.1 [C-2(I)], 123.3 [C-1(I)], 135.1 [C-4(IV)], 136.3 [C-4(III)], 136.7 [C-4(V)], 144.4 [C-3(I)], 144.7 [C-5(V)], 145.2 [C-5(III)], 165.9 [C-7(II)], 166.3 [C-7(I)], $167.2[\mathrm{C}-7(\mathrm{~V})], 167.6$ [C-7(III)], 170.3 [C-7(IV)]

\section{Castalagin:}

${ }^{1} \mathrm{H}$ NMR (600 MHz; D2O acidified with d-TFA): $\delta 4.24$ [d, 1H, J=12.8 Hz, H-6], 5.00 [dd, 1H, J=2.1, $12.7 \mathrm{~Hz}, \mathrm{H}-6$ ], 5.10 [d, 1H, J=6.4 Hz, H-3], 5.16 [t, 1H, J=7.1 Hz, H-2], 5.21 [d, 1H, J=4.7 Hz, H-4], 5.59 [d, 1H, J=7.3 Hz, H-5], 5.78 [d, 1H, J=4.8 Hz, H-1], $6.80[\mathrm{~s}, 1 \mathrm{H}], 6.86[\mathrm{~s}, 1 \mathrm{H}], 7.00[\mathrm{~s}, 1 \mathrm{H}]$

${ }^{1} \mathrm{H}$ NMR (600 MHz; [D 6 -acetone: $\mathrm{D}_{2} \mathrm{O}$ 8:2): $\delta 4.00$ [d, 1H, J=12.6 Hz, H-6], 4.96 [dd, $1 \mathrm{H}, \mathrm{J}=1.0,7.1 \mathrm{~Hz}, \mathrm{H}-3$ ], 4.98 [dd, 1H, J=1.4, 4.7 Hz, H-2], 5.00 [d, 1H, J=2.6, $13.0 \mathrm{~Hz}$, $\mathrm{H}-6], 5.15$ [t, 1H, J=7.3 Hz, H-4], 5.53 [dd, 1H, 1.1, J=7.5 Hz, H-5], 5.66 [d, 1H, J=4.7 Hz, H-1], 6.60 [s, 1H, H-2(IV)], 6.74 [s, 1H, H-6(III)], 6.76 [s, 1H, H-6(V)]

${ }^{13} \mathrm{C}$ NMR (151 MHz; [ $\left.\mathrm{D}_{6}\right]$-acetone: $\mathrm{D}_{2} \mathrm{O}$ 8:2; partial assignment based on HMQC and HMBC correlations): $\delta 65.1$ [C-6], 66.0 [C-3], 66.8 [C-1], 69.0 [C-4], 70.9 [C-5], 73.8 [C-2], 107.3 [C-2(IV)], 108.0 [C-6(V)], 108.5 [C-6(III)], 114.2 [C-2(III)], 114.8 [C6(IV)], 115.7 [C-2(I)], 115.7 [C-2(V)], 121.7 [C-1(I)], 135.8 [C-4(IV)], 136.3 [C-4(III)], 136.4 [C-4(V)], 143.3 [C-3(I)], 145.0 [C-5(V)], 145.2 [C-5(III)], 145.2 [C-3(IV)], 164.5 [C-7(I)], 165.4 [C-7(II)], 166.5 [C-7(V)], 167.1 [C-7(III)], 169.1 [C-7(IV)] 
VITA

ALLISON L. ADONIZIO

October 3, 1978

Born, Kingston, Pennsylvania

1996-2000

Rensselaer Polytechnic Institute

B.A., Biochem/Biophysics,

May 2000

Magna cum laude

Troy, New York

Exchange Year 1998-1999

Swiss Federal Institute of Technology

(ETH)

Zurich, Switzerland

Biology/Neuroscience

June 2000-June 2002

Yale University

New Haven, Connecticut

Graduate coursework in Molecular

Biophysics and Biochemistry

Laboratory Technician (Molecular, Cellular, and Developmental Biology)

Aug 2002 - March 2008

Florida International University

Miami, FL

Doctoral Candidate in Biology

Sep 2006 - May 2007

Harvard University

Boston, MA

Research Associate (Molecular Biology)

May 2007 - Aug 2007

Florida International University

Miami, FL

Lab Course Instructor

(General Microbiology) 


\section{PUBLICATIONS AND PRESENTATIONS}

Adonizio, A., Leal, S.M., Ausubel, F.M., and K. Mathee. 2008. Attenuation of Pseudomonas aeruginosa virulence by medicinal plants in a Caenorhabditis elegans model system. Journal of Medical Microbiology. In Press.

Adonizio, A., Kong, K-F, and K. Mathee. 2008. Inhibition of quorum sensing-controlled virulence factor production in Pseudomonas aeruginosa by south Florida plant extracts. Antimicrobial Agents Chemotherapy. 52(1):198-203.

Adonizio, A. L., K. Downum, B. C. Bennett, and K. Mathee. 2006. Anti-quorum sensing activity of medicinal plants in southern Florida. Journal of Ethnopharmacology. 105(3):427-35.

"Identification of quorum sensing inhibitors in south Florida medicinal plants: an understudied aspect of efficacy". Joint meeting of the Society for Economic Botany (45th Annual Mtg.) and the International Society for Ethnopharmacology (9th International Congress). June 2004. Kent, UK. Awarded Best Presentation in Ethnopharmacology

"Anti-quorum sensing activity of south Florida medicinal plants" Poster Presentation. American Society for Microbiology: Microbial Pathogenicity and Host Response. September 2005. Cold Spring Harbor Labs, NY

"Anti-quorum sensing agents from south Florida medicinal plants and their potential to attenuate Pseudomonas aeruginosa pathogenicity." MBRS Comparative Immunology Symposium. March 2006. Miami, FL. Awarded Second Place

"Anti-quorum sensing compounds from plants: expanding our view of antibacterial efficacy." 46th Annual Meeting of the Society for Economic Botany. June 2006. Fort Worth, TX

"Anti-quorum sensing activity of six south Florida medicinal plants and their ability to inhibit Pseudomonas aeruginosa pathogenicity". 47th Annual Meeting of the Society for Economic Botany. June 2006. Chiang Mai, Thailand

"The ability of the medicinal plant Conocarpus erectus (Combretaceae) to inhibit bacterial virulence through interruption of cell-cell communication" Poster Presentation. American Society for Microbiology 106th General Meeting. May 2006. Orlando, FL.

Awarded Corporate Student Travel Grant

\section{AWARDS}

- MBRS RISE Fellow- Awarded May 2006, beginning August 2006-2008

- Student Training Grant - Cystic Fibrosis Foundation - Awarded 3 years : 2005-2008

- Student Summer Research Award - FIU (MBRS RISE Program) - Awarded May 2005

- Ralph Sanchez Scholarship- Minority student grant - Awarded Jan 2005

- Presentation Award- MBRS Comparative Immunology Symposium - Feb 2005

- Corporate Student Travel Grant - American Society of Microbiology - May 2006 